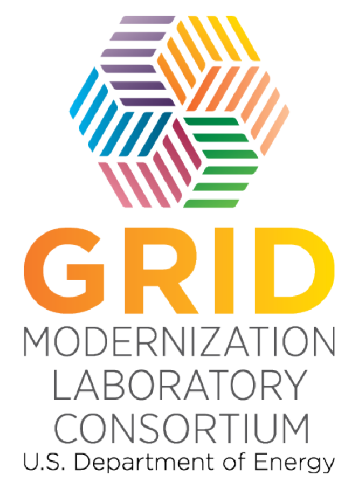

\title{
Fast Grid Frequency Support from Distributed Energy Resources
}

\section{March 2021}

Andy Hoke

Rasel Mahmud

Austin Nelson

Dinesh Pattabiraman

Marc Asano

Dean Arakawa
Brian Pierre

Mohamed Elkhatib

Jin Tan

Vahan Gevorgian

Chris Antonio

Earle Ifuku 


\section{Fast Grid Frequency Support from Distributed Energy Resources}

\author{
Andy Hoke \\ Rasel Mahmud \\ Austin Nelson \\ Dinesh Pattabiraman \\ Marc Asano \\ Dean Arakawa
}

\author{
Brian Pierre \\ Mohamed Elkhatib \\ Jin Tan \\ Vahan Gevorgian \\ Chris Antonio \\ Earle Ifuku
}

March 2021 


\section{Executive Summary}

This report describes research related to electric power system frequency support from invertercoupled distributed energy resources (DERs). ${ }^{1}$ This research was initiated under the U.S. Department of Energy's Grid Modernization Laboratory Consortium (GMLC) and also contains work funded through a Cooperative Research and Development Agreement with Hawaiian Electric. The purpose of this report is to summarize the findings of simulations, hardware testing, power hardware-in-the-loop (PHIL) simulation, and analysis examining the effects of DER frequency support on the Oahu power system. This report also includes recommendations for consideration in the use of DER grid frequency support functionality, and related topics.

Note that a previous GMLC Technical Report ${ }^{2}$ from this project also provided specific recommendations for the activation of one specific grid frequency support function, frequencywatt control, for overfrequency events in Hawaii. Those recommendations included recommended frequency-watt control settings, which have since been approved by the Hawaii Public Utilities Commission on October 20, 2017, for system-wide activation. ${ }^{3}$ This report builds on the previous report by considering not only overfrequency events but also underfrequency events, and by considering distributed energy storage systems in addition to photovoltaic (PV) systems.

This report contains the following:

- An updated description of the current state of the art of distributed inverter-based frequency support

- Lab test results of presently available distributed-scale PV and storage inverters performing frequency-watt control

- A mathematical (eigenvalue) analysis of the frequency stability of power systems containing both conventional generation and high levels of inverter-based resources with frequency support capability

- Bulk power system simulation results of the Oahu power system for 2019 scenarios, studying

- Frequency support from distributed inverters

- Transmission fault events, including the responses of distributed inverters

- $\quad$ Power hardware-in-the-loop (PHIL) test results of PV and storage inverters with frequency-watt control enabled

- Conclusions and recommendations related to activation of frequency-watt control in distributed PV inverters

\footnotetext{
${ }^{1}$ As used in this report, the term "DERs" includes only distribution-connected resources, and does not include loads.

${ }^{2}$ NREL/TP-5D00-68884: https://www.nrel.gov/docs/fy17osti/68884.pdf

${ }^{3}$ Decision and Order 34924, Hawaii Public Utilities Commission, October 20, 2017
} 
- Recommendations on how to implement frequency-watt control and the grid service known as fast frequency response $(\mathrm{FRR})^{4}$ such that they are mutually compatible

- An analysis of the impact of inverter enter service criteria (also known as Return to Service) settings on DER energy production and a discussion of the impacts of those settings on grid restoration following outages.

Brief summaries of each of these topics are presented in this executive summary.

Because the vast majority of DERs are not connected to a utility communication network, and because of the need for very fast response times, this report considers autonomous inverter control functions that do not require communications. By pre-programming DER inverters to respond autonomously to local conditions, it is feasible for large numbers of distributionconnected inverters to support grid frequency without requiring a communications network or standardized communication protocols. A prerequisite for such functions is that the inverters be designed to remain connected to the grid during (or "ride through") frequency disturbances. The most commonly available inverter-based frequency support function is frequency-watt control, also known as frequency droop control. The function itself is similar to governor droop control of synchronous generators in that the inverter measures the AC grid frequency present at its terminals and responds by modulating its power following a droop curve designed to help move the frequency back towards its normal range. ${ }^{5}$ This frequency-watt droop functionality, also referred to as primary frequency response (PFR), is a fundamental stabilizing function used in all interconnected power grids, and is crucial to ensuring stable bulk power system operations.

Frequency-watt control of distributed $\mathrm{PV}$ inverters is of interest because as the cumulative installed capacity of distributed PV becomes large enough that it can affect the AC grid frequency, it would be beneficial for distributed PV systems to be operated in a way that minimizes negative impacts on frequency stability, and if possible has a beneficial impact. The Hawaiian island power systems have most certainly reached the point where distributed PV impacts must be accounted for by bulk power system operators: distributed PV capacity is around half of peak system load. Hence, Hawaii was the first location in the U.S. to require distributed PV systems to ride through a wide range of frequency conditions (though several other states have since followed suit). Inverters with frequency-watt control enabled go beyond simply riding through frequency disturbances by actively adjusting their power output to help stabilize system frequency, similar to the droop response of synchronous generators.

Most residential- and commercial-scale PV and storage inverters sold today are capable of frequency-watt control for overfrequency events, which require a reduction in output power to mitigate excess generation. PV inverters sold today are not generally designed to be capable of responding to underfrequency events by increasing their output power; this is certainly possible, but it would require the inverter to operate below the maximum available power, a major change

\footnotetext{
${ }^{4}$ Note that in the Hawaii context, and in some other specific locations, FFR is defined as a specific class of grid services characterized by a step-like response in power output. In other contexts, the term "fast frequency response" and the acronym "FFR" are defined more broadly to mean any response that rapidly modulates energy injection into (or absorption from) a power system to help arrest a frequency event, regardless of the specifics of how the amount of energy injected or absorbed is determined. For the purposes of this report, FFR refers to the Hawaii grid services.

${ }^{5}$ Abnormal frequency events occur due to a mismatch in generation and load. Generators with droop response enabled autonomously change their power output in inverse proportion to the deviation in frequency, which helps re-balance generation and load (but does not restore frequency all the way back to the nominal value).
} 
from current operating scenarios. At the time of the research described here, most distributedscale storage inverters also were not yet capable of responding to underfrequency events by increasing power output (or reducing power consumption).

However, the newly-updated IEEE Standard 1547-2018 requires all DERs to be capable of frequency-watt control for both overfrequency and underfrequency events. ${ }^{6}$ 1547-2018 does not require DERs to maintain power headroom (so PV systems can still operate at their present maximum available power), but if a DER is operating below its maximum available power when an underfrequency event occurs, the DER is required to respond by increasing power. Also note that unlike other regulation functions required by 1547 , which can be enabled or disabled, there is no provision in 1547-2018 to disable frequency-watt control, re-enforcing the idea that the broad 1547 stakeholder group views frequency-watt droop as a baseline functionality necessary for stable grid operation.

Frequency-watt droop response (PFR) is also required of all new bulk power system resources, including inverter-based resources, as a precondition of interconnection: Federal Energy Regulatory Commission (FERC) Order 842, issued in February 2018, "require[s] that all new generating facilities install, maintain and operate a functioning governor or equivalent controls as a precondition of interconnection" in recognition that PFR is "an essential service in ensuring the reliability and resilience of the North American Bulk Power-System". Hence, once 15472018 is widely adopted, nearly all mainland U.S. inverter-based resources will be required to provide frequency-watt droop (i.e., PFR).

Now that both California and Hawaii require frequency-watt control activation (starting in February 2019 in California), most manufacturers of distributed PV inverters are testing the functionality as part of their Underwriters' Laboratories 1741 Supplement A (UL 1741 SA) certification. California's Rule 21 interconnection requirements do not specify the form of the frequency-watt function in detail, but Hawaii's UL 1741 SA Source Requirements Document (SRD) does, and the form specified in the SRD is in alignment with IEEE 1547-2018. ${ }^{7}$ In coming years, testing of frequency-watt control and other advanced DER functionality will be implemented using the test procedures in the newly published IEEE Standard 1547.1-2020.

Lab tests conducted at the National Renewable Energy Laboratory (NREL) as part of this project have confirmed that presently available PV and storage inverters can perform the frequency-watt function but that the form of the function varies between inverters. Lab tests have also confirmed that most inverters can respond very quickly to frequency changes, modifying their output power on a sub-second time scale. This is an important finding for lowinertia grids such as those in Hawaii because frequency events happen very quickly on island grids with no interties to other electric power systems, so any response must occur very quickly to be effective in mitigating a frequency event. The inverters tested under this project include two models of microinverter, three models of residential-scale PV string inverter, and one residential-scale storage inverter. At the time of testing (2016 and 2017), all of the inverters were able to provide reliable responses to overfrequency events, but only one (not the storage inverter, interestingly) was able to increase power in response to underfrequency events. This is likely a control software limitation, rather than a physical hardware capability limitation;

\footnotetext{
${ }^{6}$ The timeline for activation of IEEE $1547-2018$ is determined by the authority governing interconnection agreements in each region, as are the specific parameter settings for functions such as frequency-watt droop control.

${ }^{7}$ When reviewing the IEEE 1547-2018 requirement for frequency droop (Clause 6.5.2.7.2), take note that a sign transcription error in Table 23 was later corrected in an errata.
} 
inverter manufacturers selling in the U.S. are expected to soon provide underfrequency response per IEEE 1547-2018, if they do not already.

For frequency-watt control of distributed inverters to be effective on the major Hawaiian island power systems, many MW of DER systems must have the function enabled. Unless existing PV systems are re-programmed or retrofitted, it will likely take several years to accumulate enough frequency-watt capable PV to have a positive impact on grid frequency stability. In addition, frequency-responsive DER generation does not replace upward or downward reserve on a oneto-one basis because of the uncertainty in the amount of distributed generation online at any given time.

Simulations of the projected 2019 Oahu bulk power system were used to evaluate the effects of DER-based frequency-responsive controls, including frequency-watt control, virtual inertial, and combinations of frequency-watt and virtual inertia. In the two scenarios considered, the light spring mix and heavy summer mix, DERs made up $51 \%$ and $42 \%$ of total instantaneous generation, respectively. Adding in utility-scale wind and solar, the renewable inverter-based resource penetration levels reach $72 \%$ and $54 \%$, respectively. These levels of system-wide DER penetration, which are already very high by the standards of most grid operators, are expected to continue to grow, with the combined peak instantaneous levels of DERs plus gridscale inverter-based resources potentially reaching $100 \%$ on some Hawaiian islands in the next few years. The simulations were performed using Hawaiian Electric's proprietary PSS/E (Power Systems Simulation for Engineering) model of Oahu, in which distributed inverters are aggregated at the nearest $46 \mathrm{kV}$ bus. The aggregations of distributed inverters in the model were modified so that a portion of them could perform the frequency-responsive controls under test. Note that simulations of both underfrequency and overfrequency events assumed higher levels of frequency responsive DERs than were present in 2019, since realistic 2019 levels provide uninteresting results (i.e. there were not enough frequency-responsive DERs online yet to positively impact system response). For most simulations of underfrequency events, some portion of the DERs were assumed to be able to respond by increasing power (i.e. either energy storage is present or the PV system is operated with power reserve), though in reality this was not the case in 2019.

The PSS/E simulations found that frequency-watt control can improve the Oahu frequency response for both overfrequency and underfrequency events, as expected. Various frequencywatt droop curve parameters were simulated. It was found that steeper droop curves reduce the peak frequency deviation from nominal during the event, but are more prone to cause oscillations in system frequency, especially when the aggregate power of inverters responding is large. It was also found that the time dynamics of the PV system response have an important impact. Specifically, if the inverters respond with a first-order time constant in the range of five to seven seconds, the system frequency is more prone to oscillations than at faster or slower response time constants. Faster responses or slower responses tend to reduce the oscillations. However, slower responses are less effective at mitigating frequency events, as judged by the maximum frequency deviation from nominal during the event. For overfrequency events, slower responses can allow the frequency to approach or reach $60.5 \mathrm{~Hz}$, at which point a large quantity of legacy and re-programmed PV trips due to overfrequency, causing an underfrequency event and possibly load shedding. Likewise for underfrequency events, slower responses led to lower frequency nadirs and increased load shedding. Within the simulated range of frequency-watt deadbands, narrower deadbands led to a slightly improved frequency response.

Bulk system simulations and hardware tests performed under this project were presented to the IEEE 1547 Working Group as evidence that very fast responses to frequency events should be 
permitted. While there were concerns in the Working Group that very fast responses could be destabilizing, it was acknowledged that for low inertia systems such as the Hawaiian islands, frequency response must be fast to be effective. As a direct outcome of the evidence presented by this project, IEEE 1547-2018 allows for sub-second frequency droop response times for the most advanced inverters (known as "abnormal operating performance Category III").

The PSS/E aggregated DER inverter models were also modified to capture inverter responses to transmission voltage events (faults), including modeling of momentary cessation and recovery. PSS/E modeling of such events does not capture distribution voltages, so it is a rough approximation of actual field conditions during faults and is unable to accurately capture unbalanced fault responses. These preliminary simulations found that transmission voltage events can lead to tripping or momentary cessation of significant quantities of DERs. As Hawaii moves towards its goal of 100\% renewable energy by 2045 (and instantaneous penetrations approaching $100 \%$ much sooner), this area urgently requires further research. DER responses to transmission events may need to be improved to ensure grid resilience when levels of inverter-based generation become very high. For example, Hawaii may need to align some DER ride-through requirements with NERC guidelines for transmission-connected generation ${ }^{8}$ rather than with the IEEE 1547-2018 requirements for distribution-connected generation.

The Oahu PSS/E model was also converted into a real-time Matlab/Simulink model and linked to a real-time SimPowerSystems model of an Oahu distribution feeder. This real-time hybrid model of the bulk power system and distribution system was used for PHIL simulations, in which hardware inverters were connected to selected locations on the simulated distribution system to validate their performance in an environment that emulates the dynamics of the Oahu power system. The hardware inverters rode through the various transients tested and provided frequency support as desired.

In the PHIL simulations, the bulk power system and the distribution system were both populated with aggregate models of PV inverters having dynamic responses designed to emulate the measured hardware inverter responses. This allowed the dynamic models of PV inverters to be validated against hardware PV inverter responses in the same PHIL tests. It also allowed the system frequency response to be observed in the presence of large aggregate ratings of frequency-watt enabled PV inverters. The PHIL test results confirmed the findings of the PSS/E simulations, adding confidence that real distribution-connected hardware inverters can successfully support the grid during overfrequency and underfrequency events. No distribution overvoltages or other undesired distribution side-effects of frequency-watt control were observed in the PHIL tests.

This study has several limitations that are important to acknowledge. First of all, it focuses on the island of Oahu, so while it is expected that these results will apply in general terms to other islands (and to other power systems in general to a lesser degree), this has not been validated. It is important to note that the island of Oahu has the highest system inertia of the four major Hawaiian island systems, but this may change as renewable resources displace traditional synchronous generators in the coming years. In addition, all models used here are approximations of the real systems they represent, so simulation and PHIL results should not be

\footnotetext{
${ }^{8}$ Specifically, the North American Electric Reliability Corporation (NERC) Inverter-based Resource Performance Task Force (IRPTF) published an Inverter-based Resource Performance Guideline requiring that transmission-connected resources ride through certain voltage deviations without ceasing to export current. The intent of this requirement, which may soon be further codified in NERC PRC-024 and/or the draft IEEE Standard P2800, is to ensure bulk power system stability and resilience.
} 
considered exact representations of specific real-world events. Certain effects are not modeled at all in this work; for example, the modeling tools used do not capture interactions that can occur between power electronic sources and conventional generators at sub-synchronous frequencies, including sub-synchronous torsional interactions with turbine shafts. Finally, as levels of inverter-based generation continue to grow, the dominant physical and control interactions of the system will change, making it necessary in many cases to transition to full electromagnetic transient (EMT) power system models that can capture interactions that occur on sub-cycle timeframes to provide better assurance of grid stability. Those EMT models are more complicated and run more slowly, so aggregation of loads and DERs will be necessary, but the accuracy of the aggregate models will need to be validated to the extent possible. Significant research or field experience will be needed to gain confidence that aggregate models of DERs sufficiently capture the potential control interactions that may occur in grids dominated by power electronic sources.

Although the study focuses on 2019 scenarios, Oahu's power system is expected to experience significant change over the coming years. Oahu does not have the diversity of renewable energy resources that Maui and Hawaii islands have, so distributed PV is expected to provide much of the renewable energy needed to meet Oahu's renewable portfolio goals. As more synchronous generators are displaced, DERs will likely need to play a larger role in stabilizing grid frequency during system disturbances. Thus, this report is just a step down a path in which continued research and analysis will be needed to determine new and improved ways that DERs (including distributed PV) can support grid frequency.

With those limitations in mind, this report makes the following conclusions and recommendations:

1. The currently available frequency-watt control function of $P V$ and storage inverters helps mitigate frequency events and may help to support the reserve planning requirements in Hawaiian Electric's system-level hosting capacity analysis, with appropriate controls and visibility in place for system operators. Assuming existing PV systems are not reconfigured, it will likely take several years to build up enough aggregate capacity of frequency-watt enabled DERs to have a significant benefit. Thus, it is recommended that new PV systems be required to activate frequency-watt for underfrequency events upon commissioning in the very near future (in addition to the existing requirement for overfrequency event response). Enabling frequency-watt control will support the continued growth in distributed PV capacity, and will help maintain alignment with IEEE 1547-2018 and California Rule 21. Remaining technical questions surrounding frequency-watt control can continue to be investigated while a base of responsive inverters is being built.

2. The form of the frequency-watt function is recommended to align with the requirements in IEEE 1547-2018 (which is also aligned with Hawaiian Electric's SRD V1.1). This form of the frequency-watt function calls for the droop curve to start from the pre-disturbance output power of the inverter (as opposed to starting from the rated power). The droop slope is a constant function of the inverter's rated power (not a function of the predisturbance power, as in the German implementation). This is the version of the function used in this work, and will provide the most effective and predictable response, improving the integration of PV and other DERs into the power system.

3. IEEE 1547-2018 defines the desired open-loop response time of the frequency-watt function for "small-signal" events (events resulting in a power change of less than 0.05 
pu), but it does not clearly define a response time requirement for larger events. This is adequate for larger interconnected power systems such as those on the U.S. mainland today, and it allows for certain types of DERs that cannot easily make large power adjustments quickly. However, on low-inertia power systems such as Hawaii's, worstcase frequency events are not small-signal events, and PV inverters are capable of fast large-signal power ramping, as demonstrated in tests described in this report. For frequency-watt control to be effective in Hawaii, the time response must be fast regardless of the magnitude of the power change (i.e. the inverters must respond quickly to large-signal events in addition to small-signal events). This will improve frequency stability and also improve the testability of the frequency-watt function. Specific response-time recommendations are included below.

4. The recommended droop slope of the frequency-watt function based on this work is between $5 \%$ and $3 \%$. A droop slope equal to that of conventional generators will allow for proportional sharing of the burden of primary frequency response between synchronous generation and distributed resources. It may also be desirable for logistical reasons to have a uniform droop slope on all major islands. A droop slope of $4 \%$ would be a reasonable and conservative choice that would align with the steeper end of droop slopes currently used by synchronous generators on the major islands. A wider range of adjustability of droop slope in both directions is recommended to allow for future adjustments as the portion of energy coming from DERs continues to rise.

5. The deadband of the frequency-watt function is recommended to be large enough that typical frequency fluctuations do not activate the function onerously, to avoid unintended impacts. The analysis presented here indicated that deadbands as small as $17 \mathrm{mHz}$ would have minimal impact on monthly PV energy generation. Because narrower deadbands provide more robust frequency response, it is recommended that the deadband be updated to $17 \mathrm{mHz}$ for both overfrequency and underfrequency. This is a change from the current Hawaii requirement of $36 \mathrm{mHz}$, but can be implemented simultaneously with addition of underfrequency response to minimize logistical challenges for DER installers and manufacturers.

6. It is recommended that the response time of the frequency-watt function, defined as the time required for an inverter to execute $90 \%$ of the power change resulting from a frequency event, should be 0.5 seconds. However, the possibility of unintended subsynchronous interactions with synchronous generators should be investigated before the aggregate power rating of frequency-responsive PV rises to a level that significantly affects grid frequency.

7. In the previous report from this project, as an interim solution it was recommended that DER manufacturers be permitted to provide inverters with whatever form of frequencywatt control they currently had available, with Hawaiian Electric's specified form of the function to be required shortly after the approval of the revision to IEEE 1547. Now that $1547-2018$ is approved and published, it is recommended that all DERs installed in Hawaii going forward provide frequency-watt response following the 1547-2018 (and Hawaiian Electric SRD V1.1) requirement. This will improve the resilience and predictability of the future Hawaii power systems.

8. Per IEEE 1547-2018, all new DERs should be required to provide frequency-watt control, even if also providing other grid services such as fast frequency response (FFR). (While some definitions of "DER" are more expansive, this report uses the IEEE 1547 
definition of DER, which includes generation and energy storage connected to distribution systems but does not include loads.) Frequency-watt control is inherently compatible with other grid services because performance is only required to the extent that active power is available. For example, just as frequency-watt control does not require a $\mathrm{PV}$ system without storage to increase power beyond its present maximum available power, a resource providing both FFR and frequency-watt control that reaches its maximum power following an FFR trigger would not be required to provide additional power to comply with frequency-watt control. The body of this report contains equations and examples intended to explain how frequency-watt control and FFR can be compatible in the same DER. Also note that for FFR resources that control loads (and do not control storage or generation), there would be no requirement to provide frequencywatt control as loads are not subject to IEEE 1547 or to Rule $14 \mathrm{H}$. For a broader treatment of various types of fast frequency control, see the NERC whitepaper "Fast Frequency Response Concepts and Bulk Power System Reliability Needs"; however, to avoid possible confusion, note that the NERC whitepaper uses a much broader definition of the term "FFR" than used in Hawaii.

9. Until it is possible using communications to remotely control DER power during system restoration from outages, tighter DER Enter Service settings would help ensure that the restored grid is stable before DERs come back online. Analysis presented in this report shows that the impact of an Enter Service Criteria setting of $59.9 \mathrm{~Hz}$ on DER energy production would be much less than $1 \%$ of annual production. Such a setting is within the range of allowable settings in IEEE 1547-2018. It is difficult to simulate a restoration event in sufficient detail to demonstrate the potential negative impacts of uncontrolled DER reconnection at $59.5 \mathrm{~Hz}$ due to a lack of standard software tools for this purpose; this would likely require a significant research effort. However, given the significant potential for negative impact, it is recommended that an enter service criteria setting of $59.9 \mathrm{~Hz}$ be implemented at least until better methods of controlling DERs during restoration become available.

10. Looking further ahead, both Hawaiian Electric and inverter manufacturers should continue to consider improving DER frequency response capabilities through research, development, and analysis. For example, it may be useful to DERs to provide responses that emulate system inertia in some form. The preferred form of the inertialike response would need to be studied and then standardized to provide a predictable system response. In addition, the stability of DER inverters in grids with very low inertia and low short-circuit capability under both normal and abnormal conditions should continue to be researched and improved. It may become preferable for DERs to avoid momentary cessation altogether, as is currently recommended for transmissionconnected inverters in the mainland U.S. It will continue to be challenging to obtain further inverter advancements given Hawaii's small market size and very high level of DER penetration relative to larger DER markets; vendors that are reluctant to develop products for Hawaii should consider that other markets may continue to follow Hawaii's lead. Future standards development efforts can assist by considering the needs of very high penetration systems like Hawaii's, thereby helping the rest of the U.S. and the world anticipate their own future challenges.

In summary, with respect to frequency-watt control, it is recommended that Hawaiian Electric, in discussions with the members of the Advanced Inverter Functions Working Group or other representatives of the DER industry, should agree to modify the Rule $14 \mathrm{H}$ interconnection tariff to activate the frequency-watt settings defined in Table 1. Figure 1 depicts a frequency-watt 
curve showing the droop slope and deadband. Equations $B$ and $C$ in Hawaiian Electric SRD V1.1 and the surrounding text describes how to apply the variables in Table 1. (IEEE 1547-2018 contains the same equations in Table 23. If using the equations in IEEE 1547-2018, carefully note the published errata, which corrects a sign error.)

Table 1: Recommended frequency-watt control settings for overfrequency events

\begin{tabular}{|c|c|c|c|}
\hline & & Default value & Range of adjustability \\
\hline Deadband (Hz) & $\begin{array}{l}\text { dbof, } \\
\text { dbuF }\end{array}$ & 0.017 & $0.017-1.0$ \\
\hline Droop slope (pu) & $\begin{array}{l}\text { KoF, } \\
\text { KuF }\end{array}$ & 0.04 & $0.02-0.07$ \\
\hline Response time (s) & $t_{R}$ & 0.5 & $0.2-3.0$ \\
\hline
\end{tabular}

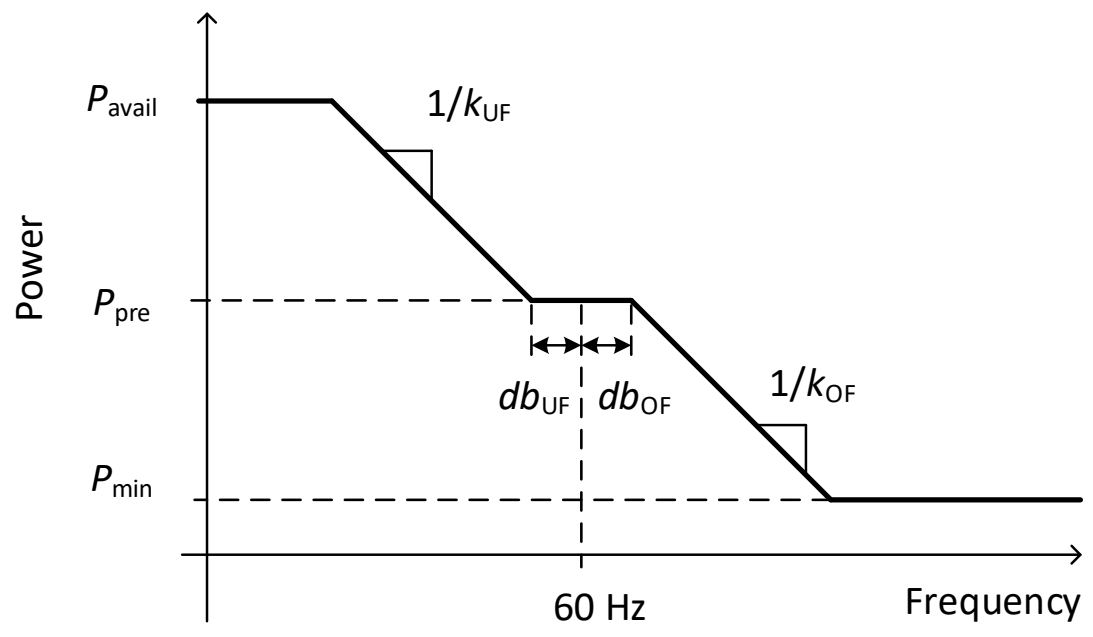

Figure 1: Frequency-watt droop curve settings 


\section{Acknowledgments}

This work was authored by the National Renewable Energy Laboratory, operated by Alliance for Sustainable Energy, LLC, for the U.S. Department of Energy (DOE) under Contract No. DEAC36-08GO28308. Funding provided in part by U.S. Department of Energy Office of Grid Modernization Laboratory Consortium. The views expressed in the article do not necessarily represent the views of the DOE or the U.S. Government. The U.S. Government retains and the publisher, by accepting the article for publication, acknowledges that the U.S. Government retains a nonexclusive, paid-up, irrevocable, worldwide license to publish or reproduce the published form of this work, or allow others to do so, for U.S. Government purposes.

This research was supported by the Grid Modernization Initiative of the U.S. Department of Energy under contract number 1.3.29 as part of its Grid Modernization Laboratory Consortium, a strategic partnership between DOE and the national laboratories to accelerate the development of technology, modeling analysis, tools, and frameworks to help enable grid modernization adoption. Additional funding for this work was provided by the Hawaiian Electric Companies.

The authors are grateful to Guohui Yuan and Merrill Smith of the U.S. Department of Energy for their valuable guidance and support.

The authors also gratefully acknowledge the inverter manufacturer project partners, Enphase Energy, Fronius USA, and SolarEdge Technologies for providing hardware, technical support, and valuable input.

Hawaiian Electric's Advanced Inverter Function Working Group provided a valuable forum for discussion of portions of this work. The authors are thankful to the Working Group members for their valuable feedback. 


\section{Acronyms and Abbreviations}

$\begin{array}{ll}\text { AGC } & \text { automatic generation control } \\ \text { DER } & \text { distributed energy resource } \\ \text { DOE } & \text { Department of Energy } \\ \text { dq } & \text { direct-quadrature } \\ \text { EMT } & \text { electromagnetic transient } \\ \text { ESIF } & \text { Energy Systems Integration Facility } \\ \text { GMLC } & \text { Grid Modernization Laboratory Consortium } \\ \text { Hz } & \text { Hertz } \\ \text { IEEE } & \text { Institute of Electrical and Electronics Engineers } \\ \text { MW } & \text { Megawatt } \\ \text { mHz } & \text { millihertz } \\ \text { MPPT } & \text { maximum power point tracking } \\ \text { NERC } & \text { North American Electric Reliability Corporation } \\ \text { NREL } & \text { National Renewable Energy Laboratory } \\ \text { PFR } & \text { primary frequency response } \\ \text { PHIL } & \text { power hardware-in-the-loop } \\ \text { PLL } & \text { phase-locked loop } \\ \text { pu } & \text { per unit } \\ \text { PV } & \text { photovoltaic } \\ \text { ROCOF } & \text { rate of change of frequency } \\ \text { S } & \text { seconds } \\ \text { SRD } & \text { Source Requirements Document } \\ \text { UDM } & \text { user-defined model } \\ \text { UL } & \text { Uniterwriters' Laboratories States } \\ \text { U.S. } & \end{array}$




\section{Contents}

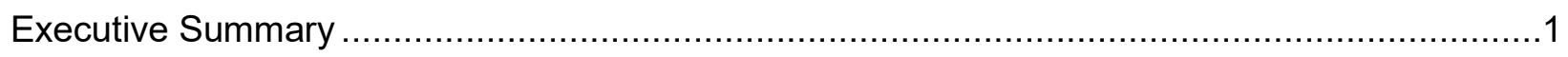

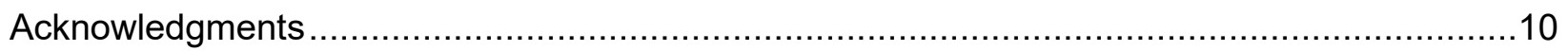

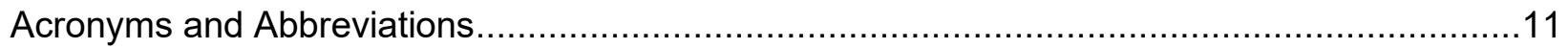

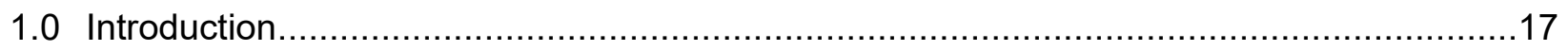

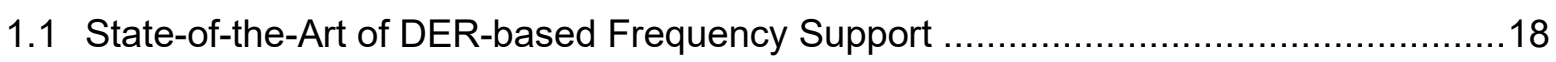

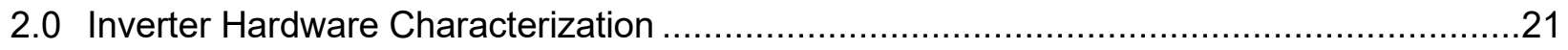

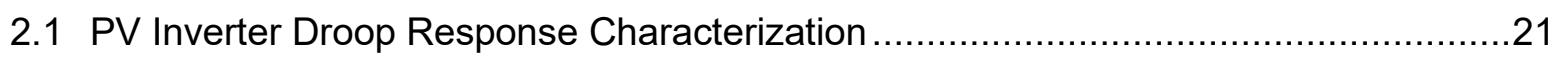

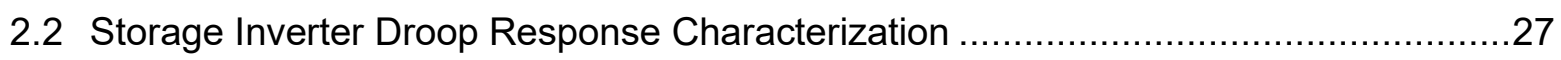

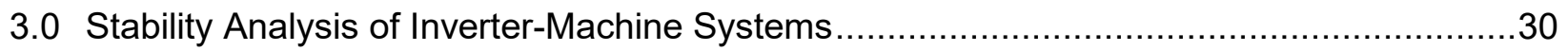

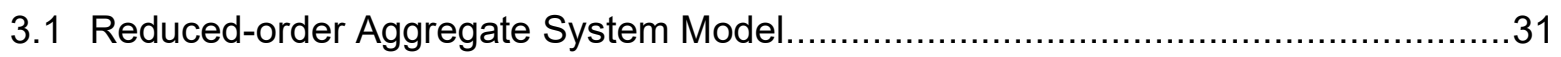

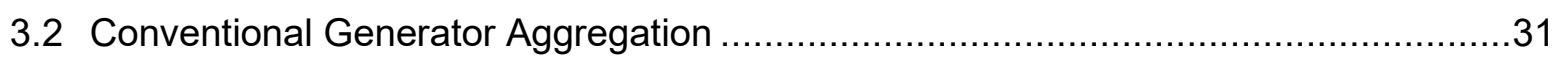

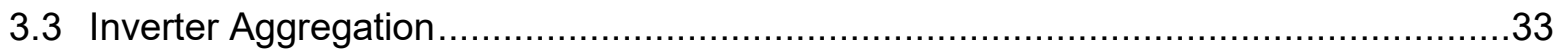

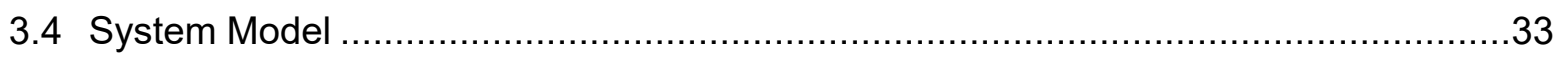

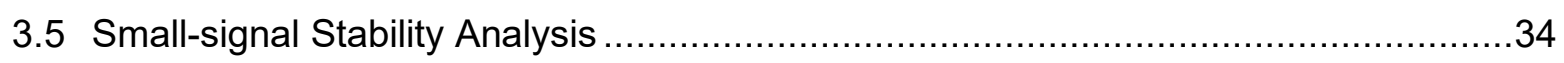

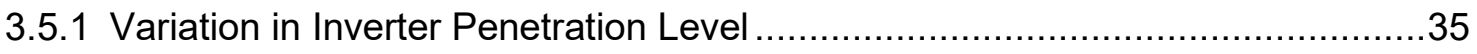

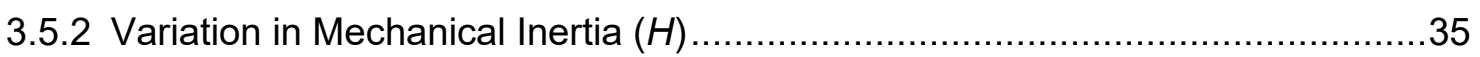

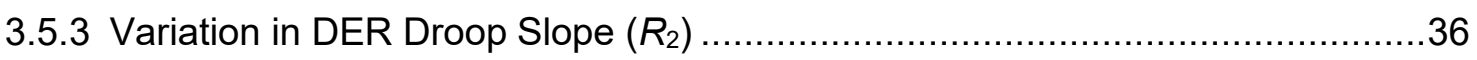

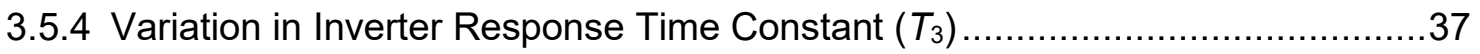

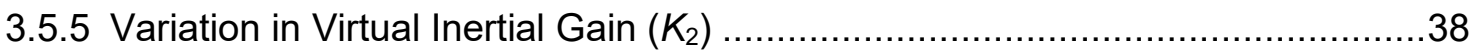

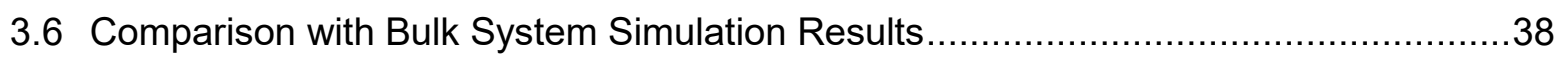

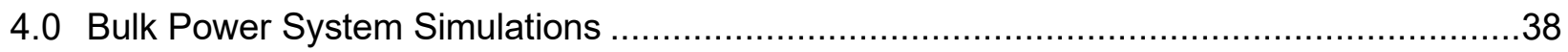

4.1 Distributed Inverter-based Generation User-defined Model for PSS/E .....................39

4.2 DER Inverter Responses to Transmission Faults ................................................. 40

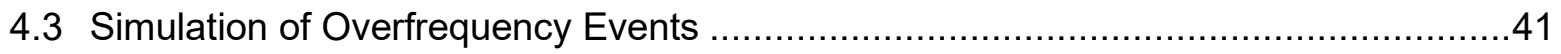

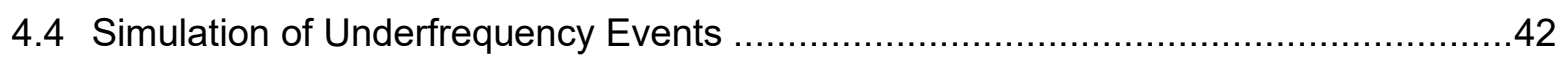

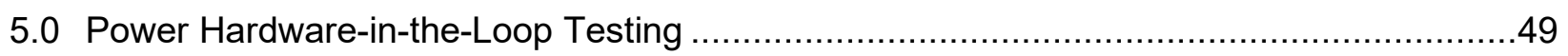

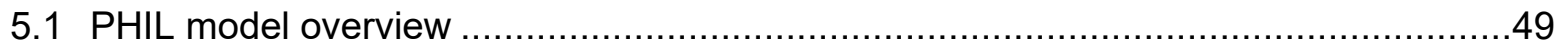

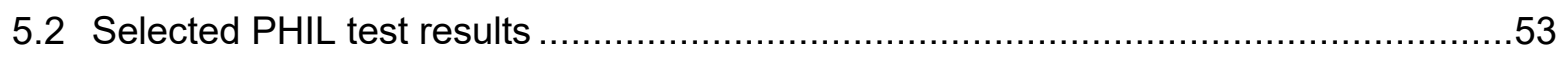

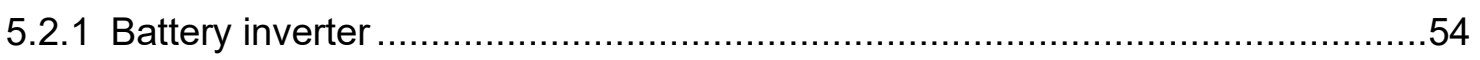

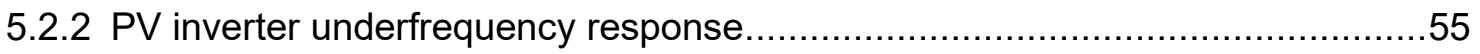

6.0 Impact of frequency-watt control on DER energy production ........................................59

7.0 Compatibility of frequency-watt control with fast frequency response (FFR) ....................61

7.1 A proposed method of performing frequency-watt control simultaneously with FFR ...63

7.2 Simulation examples of simultaneous frequency-watt control and FFR .....................64

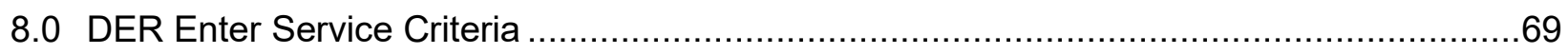

8.1 Impact of Enter Service Criteria on DER Energy Production .................................69 
8.2 Impact of Enter Service Criteria on System Restoration ......................................79

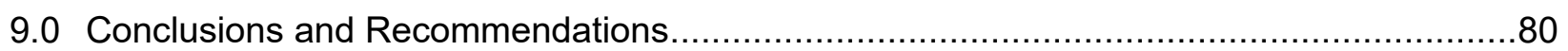

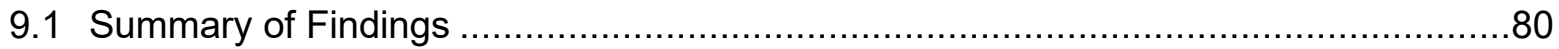

9.2 Challenges of DER-Based Frequency Support................................................ 80

9.3 Recommendations for Activation of Frequency-Watt: Default Settings and Ranges of

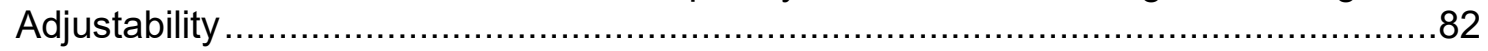

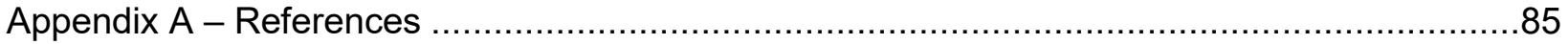




\section{Figures}

Figure 1: Frequency-watt droop curve settings.

Figure 2: Qualitative depiction of a frequency event showing frequency response time scales.

While this figure depicts an underfrequency event, the same time scales apply to

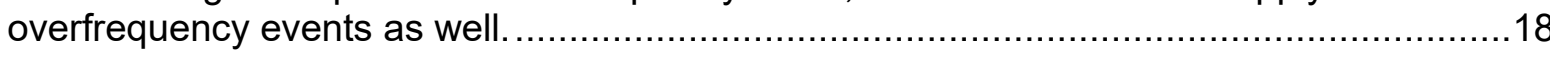

Figure 3: A frequency-watt droop curve ..................................................................... 19

Figure 4: Example of Inverter 2 frequency-watt time response ...........................................22

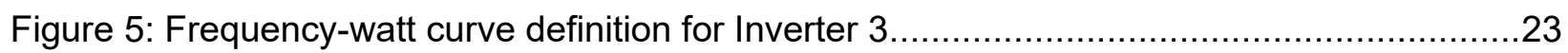

Figure 6: Inverter 3 frequency-watt test with power reserved to allow for underfrequency response.

Figure 7: Comparison of Inverter 3 frequency-watt measured characteristic to programmed

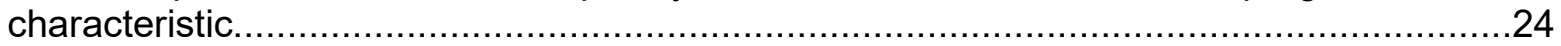

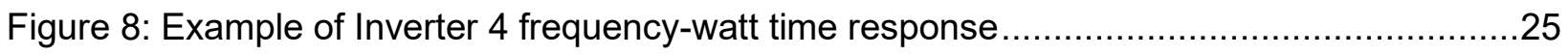

Figure 9: Inverter 4 steady-state frequency-watt response .............................................26

Figure 10: Inverter 5 time domain frequency-watt response ...........................................26

Figure 11: Inverter 5 steady-state frequency-watt response ...........................................27

Figure 12: Frequency-watt response of Inverter 6 (storage inverter) while charging................28

Figure 13: Steady-state frequency-watt response of Inverter 6 (storage inverter) while

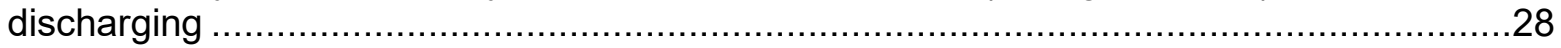

Figure 14: F-W response of StorEdge inverter during discharging ..................................29

Figure 15: Time domain droop response of Inverter 6 while discharging, compared to simulation of aggregate response of multiple inverters of this type. ............................................ 30

Figure 16: One-line diagram of reduced-order network with aggregated sources....................31

Figure 17: Aggregate conventional generator model used for small-signal stability analysis. ....32

Figure 18: Comparison of frequency responses of the aggregated generator model and the full governor-only model.

Figure 19: One-line diagram of reduced-order network with aggregated sources....................33

Figure 20: Eigenvalue trajectories (left) and time domain responses (right) for variation in

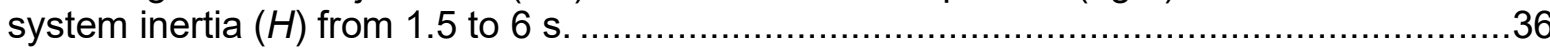

Figure 21: Eigenvalue trajectories (left) and time domain responses (right) for variation in in DER droop $\left(R_{2}\right)$ from $2 \%$ to $8 \%$.

Figure 22: Eigenvalue trajectories (left) and time domain responses (right) for variation in inverter response time constant $\left(T_{3}\right)$ from 0.3 to $3 \mathrm{sec}$.

Figure 23: One-line diagram of reduced-order network with aggregated sources.....................38

Figure 24: Distributed inverter PV model including frequency-watt function .........................40

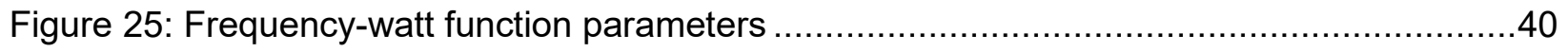

Figure 26: Baseline underfrequency event with no frequency-responsive DERs ....................45

Figure 27: Total load during baseline underfrequency event ........................................45

Figure 28: Total distributed PV power during baseline underfrequency event .......................46 
Figure 29: Frequency response with 4\% DER droop slope and varying levels of DER reserve power

Figure 30: Comparison of DER power levels for frequency events with $4 \%$ DER droop slope and varying levels of DER reserve power ...

Figure 31: Total distributed PV power for frequency events with 4\% DER droop slope and varying levels of DER reserve power .

Figure 32: Total load for frequency events with 4\% DER droop slope and varying levels of DER reserve power.

Figure 33: Real-time model of Oahu bulk system and distribution system for PHIL.................50

Figure 34: Oahu bulk power system frequency dynamic model........................................51

Figure 35: PHIL test of storage inverter response to overfrequency ..................................55

Figure 36: PHIL underfrequency events with varying droop slopes ....................................57

Figure 37: PHIL underfrequency events with varying inverter response times........................58

Figure 38: PHIL underfrequency events with varying deadband and droop slope ...................58

Figure 39: PHIL underfrequency events with varying amounts of available PV power held in

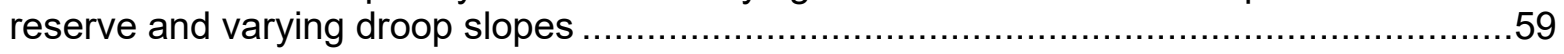

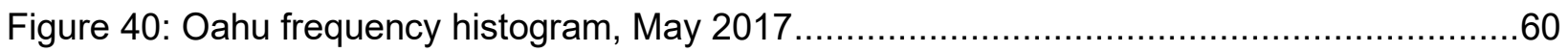

Figure 41: Frequency-watt (f-W) and FFR control regions.........................................6 62

Figure 42: Simulation of DER underfrequency response while performing both frequency-watt control and FFR with power reserve equal to the FFR nominated capability.

Figure 43: Close up of initial portion of Figure 42. Note that a significant portion of the power reserve is used up before the FFR response is triggered.

Figure 44: Simulation of DER underfrequency response while performing both frequency-watt control and FFR with power reserve exceeding the FFR nominated capability......

Figure 45: Close up of initial portion of Figure 44 illustrating interplay between FFR and frequency-watt control.

Figure 46: Illustration of an unable-to-reconnect window showing recorded grid frequency, inverter reconnection timer, and duration of unable-to-reconnect window. ....

Figure 47: Unable-to-reconnect windows during a typical month (March 2017, Hawaii Island)..71

Figure 48: Unable-to-reconnect windows during the worst-case month (June 2017, Maui) ......72

Figure 49: A closer view of unable-to-reconnect windows for the circled period in Figure 48 showing that what appears to be a single very long unable-to-reconnect window is actually several windows with opportunities in between for any offline DERs to reconnect.

Figure 50: Percentages of time covered by unable-to-reconnect windows by hour of day for Oahu.

Figure 51: Percentages of time covered by unable-to-reconnect windows by hour of day for Maui.

Figure 52: Percentages of time covered by unable-to-reconnect windows by hour of day for Hawaii Island. 


\section{Tables}

Table 1: Recommended frequency-watt control settings for overfrequency events ..................9

Table 2: Single-machine Aggregate Model Parameters. .................................................3

Table 3: Baseline Generator Parameters for Small Signal Stability Study ............................34

Table 4: Baseline Generator Parameters for Small Signal Stability Study .............................35

Table 5: Oahu 2019 Light Spring Case Generation Mix ...................................................42

Table 6: Oahu 2019 Heavy Summer Case Generation Mix .............................................43

Table 7: Frequency-watt control base model parameters for PSS/E simulations.....................44

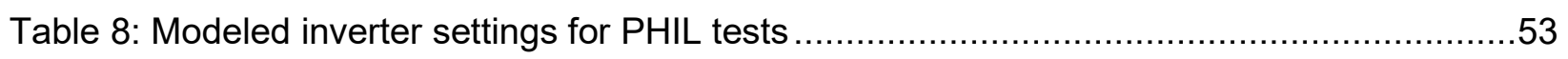

Table 9: Estimated impact of frequency-watt control on PV energy production ......................60

Table 10: Enter service criteria from IEEE 1547-2018 ...............................................69

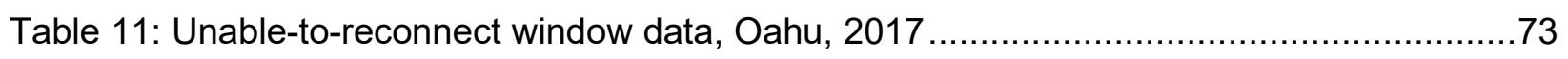

Table 12: Unable-to-reconnect window data, Maui, 2017 ..........................................74

Table 13: Unable-to-reconnect window data, Hawaii Island, 2017 .......................................74

Table 14: Lost PV energy production due to proposed enter service criteria, Oahu, 2017 ........77

Table 15: Lost PV energy production due to proposed enter service criteria, Maui, 2017.........78

Table 16: Lost PV energy production due to proposed enter service criteria, Hawaii Island, 2017 79

Table 17: Recommended frequency-watt control settings for overfrequency and underfrequency events. 


\subsection{Introduction}

As increasing amounts of non-synchronous generation such as solar photovoltaic (PV) systems and battery energy storage (BES) systems are interconnected with electric power systems, those generators displace some synchronous generators. Those synchronous generators stabilize grid frequency on the fastest time scales through their rotational inertia and primary frequency response (PFR), so displacement of synchronous generation can result in reduced frequency stability. The state of Hawaii leads the nation in the proportion of its energy that is provided by distributed PV. This, in combination with its small size and geographic isolation, is forcing Hawaii utilities to confront grid reliability issues associated with high PV penetrations sooner than mainland U.S. utilities.

Hawaiian Electric already requires that new distributed energy resources (DERs) such as PV continue operating during (or "ride through") a wide range of frequency events to avoid exacerbating frequency disturbances. However, as levels of distributed PV have continued to rise and as distributed energy storage systems begin to become common, it is desirable for DERs to not just avoid exacerbating disturbances, but also to actively help maintain frequency stability [1]. DER inverters can do this by quickly modulating their power output in response to frequency changes they sense on the grid [2], [3].

A previous report from this project team examined a subset of the DER frequency response space: overfrequency response from distribution-scale PV inverters using frequency-watt droop control [4]. It concluded that the droop response in presently-available inverters was fast and reliable enough to help mitigate overfrequency events. The results were used as technical justification to require activation of frequency-watt control, an action the Hawaii Public Utilities Commission approved in October 2017.

This report expands on the previous report by examining fast frequency support (including but not limited to frequency-watt droop control) by both solar and storage inverters in response to both underfrequency and overfrequency events. The dynamics of such control are crucial - it must be fast enough to respond to the rapid frequency events that are characteristic of Hawaii's relatively low-inertia grids, but it must not cause further instability.

Laboratory tests of presently available PV and storage inverters' frequency-watt functionality were conducted both to validate the functionality and to characterize the inverters' dynamic responses. Simplified models of the inverters' frequency-watt responses were developed and used to simulate the effect of large aggregations of such inverters during Oahu frequency events. In addition, a power hardware-in-the-loop (PHIL) test apparatus was developed to capture Oahu's bulk system frequency dynamics as well as the electromagnetic dynamics of an Oahu distribution feeder. The PHIL model was populated with modeled PV systems, and two hardware PV inverters were connected to it, allowing them to be tested in an environment that emulates the voltage and frequency dynamics seen by distributed PV inverters on Oahu. This combination of conventional lab testing, simulations, and PHIL testing was used to investigate the effect of various frequency-watt control settings on Oahu frequency response.

This report is part of a larger project that examines the ability of distributed PV and energy storage to support grid frequency on the inertial and PFR time scales, and also investigates and develops new methods of DER frequency support. The frequency response control time scales are depicted in Figure 2, which is adapted from [5]. 


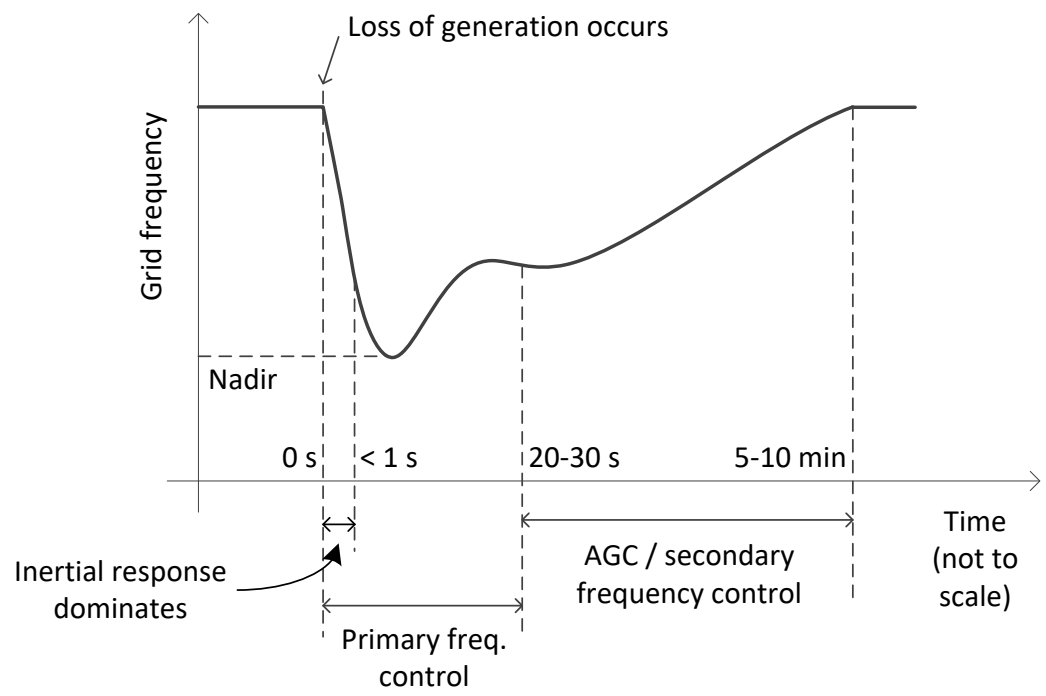

Figure 2: Qualitative depiction of a frequency event showing frequency response time scales. While this figure depicts an underfrequency event, the same time scales apply to overfrequency events as well.

\subsection{State-of-the-Art of DER-based Frequency Support}

PV and other DERs are not currently required to provide frequency support most areas in the U.S. outside of Hawaii and California, though they are in some other countries, notably Germany [6]. However, most PV and storage inverters available in the U.S. include the ability to reduce power output in response to overfrequency events following a frequency-watt droop curve, and the recently published IEEE 1547-2018 requires all DERs to provide frequency droop control for both underfrequency and overfrequency events [7]. ${ }^{9}$ This function is similar to the governor droop control often enabled in synchronous generators, though inverters can typically respond much more quickly. Presently available PV inverters for residential- and commercialscale applications typically do not include the ability to increase power in response to underfrequency events, and many storage inverters do not either. However, this is expected to change as inverter manufacturers update their products in response to 1547-2018 and California Rule 21 requirements.

Providing any sort of frequency response requires the DER inverters to be configured to remain online during (ride through) frequency events, as is the case in Hawaii, California, and Independent System Operator of New England (ISO-NE) territory but not yet in most of the U.S. However, ride-through requirements are beginning to proliferate as grid operators realize that aggregate DER power levels either are or will soon be enough to affect bulk system stability in many areas [8].

Frequency-watt control is an autonomous function that does not rely on communications for its operation. The inverter measures the AC grid frequency at its terminals and responds by modulating its output power to follow a droop curve such as the one shown in Figure 3.

\footnotetext{
${ }^{9}$ Note that IEEE 1547-2018 does not require DERs to maintain power reserve for underfrequency response.
} 


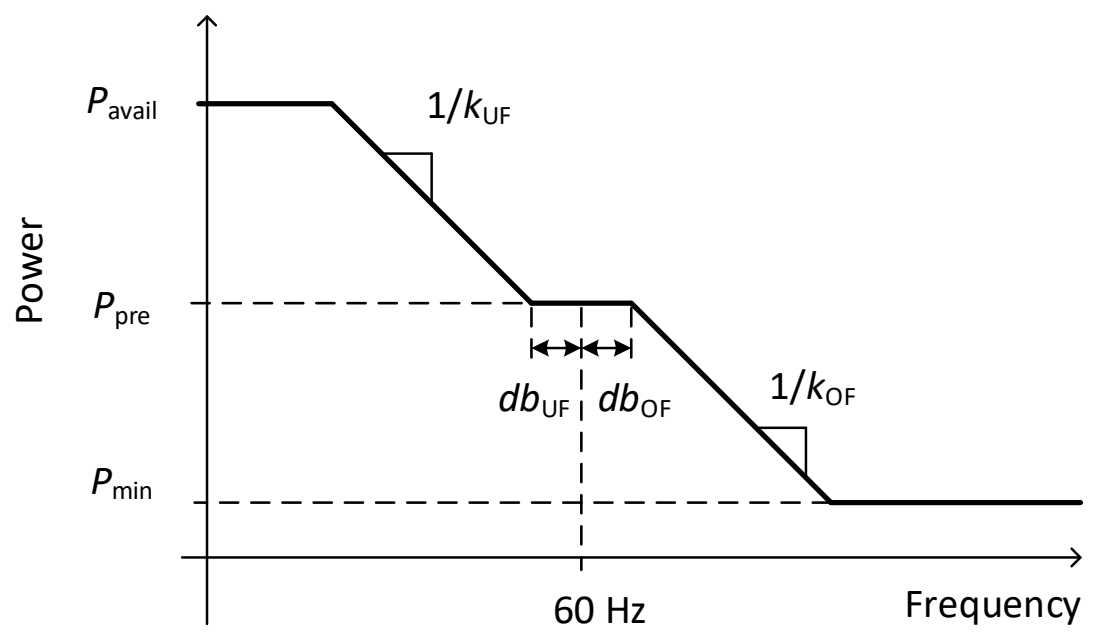

Figure 3: A frequency-watt droop curve

There are several possible forms the frequency-watt function can take. For example, the starting point of the droop curve can be the pre-disturbance operating power, or it can be the rated power. IEEE 1547-2018 requires the droop curve to start from the pre-disturbance operating power (sometimes called $P_{\text {pre }}$ ), which provides power system operators with a predictable response without knowledge DER operating power levels.

Underwriter's Laboratories (UL) Standard 1741 Supplement SA (UL 1741 SA) [9], published in September 7, 2016, provides a standardized test procedure for frequency-watt control. UL 1741 SA is largely agnostic to the form and parameters of the frequency-watt function; it relies on each utility to provide a Source Requirements Document (SRD) that specifies the details of the desired grid support functions. In September 2017, Hawaiian Electric published its revised SRD [10], which specifies frequency-watt control details that are aligned with those in IEEE 15472018. IEEE 1547's companion test standard, IEEE 1547.1-2020, recently published updated test procedures for frequency-watt control and is expected to be widely implemented soon, but those provide in UL 1741 SA are sufficient for the present.

Other forms of DER frequency support that have been proposed include:

- Virtual inertial response ${ }^{10}$, in which the inverter modulates power in response to the rate of change of frequency (ROCOF) rather than the absolute frequency deviation. Inertial response is permitted by 1547-2018 but is not required, and no specifications are provided. It is important to note that in the context of inverter-based generation, virtual inertial response is not the same as physical inertia; instead it is a control action that emulates the inertial response of rotating generators, so is not instantaneous. The response can also be modified to not exactly emulate inertia (for example to avoid pushing back when frequency begins to recover). The authors are not aware of any residential or small commercial-scale inverters advertising inertial response capability at the time of writing. This is likely due to software limitations rather than hardware - most

\footnotetext{
${ }^{10}$ Virtual inertial response is sometimes also referred to as virtual inertia or synthetic inertia. It may also refer to any response that occurs on the inertia time scale. However, it is recommended to restrict its use to control schemes that emulate synchronous machine inertia response.
} 
inverters have not been programmed for inertial response due to a lack of demand for it at present.

- Fast frequency response (FFR ${ }^{11}$, in which a DER rapidly modulates its output power following a pre-determined step change command in response to a trigger such as frequency or ROCOF surpassing a pre-set level or a communicated signal. Hawaiian Electric does have an FFR program in operation, but none of the inverters tested under this project were capable of FFR. FFR can also be provided by modulating load power.

- Automatic generation control (AGC, also referred to as secondary frequency regulation) and other longer time-scale controls. While droop control, virtual inertia, and FFR all generally operate within the inertial and PFR time scales (sub-second to a few tens of seconds), DERs can also be controlled to operate on longer time scales [11], [12]. Often these longer time-scale controls include communications so are not well-suited for today's DERs. For example, AGC requires continuous reliable communication with the utility.

This report examines virtual inertial response through simulations and theoretical analysis. It does not examine FFR in detail; some references on FFR are available elsewhere [13], [14]. This report also does not examine controls that operate on the AGC time scale or longer.

While frequency-watt and other fast controls do not rely on communications, a communication pathway could be used if desired to remotely change settings. At the time of this writing, most inverter manufacturers support some form of communications, but there is no single communications protocol that is supported by all manufacturers, and not all DERs are connected to a communications network. Thus there would be no uniform way to remotely configure and activate the frequency-responsive controls for many DERs from many different manufacturers. This project focuses on the power functionality with the assumption that if frequency-watt control is enabled in the near future, each PV system will be configured to provide it at the time of installation.

While frequency-watt control of inverters is similar to conventional generator governor droop response (typically used for primary frequency response, or PFR), it differs in several key ways:

1. Most DERs are PV systems without storage, and as most PV inverters are operating at their maximum available power most of the time, so while downward response is readily available, upward response is only available from DERs with storage or when operating below maximum available power.

2. An inverter's response can be much faster than a conventional generator's response if desired. While synchronous generators typically have power response time constants on the order of a few seconds, inverters can typically adjust power in less than one second if desired.

3. PV systems are coupled to the grid through power electronics and do not have rotational inertia, so they do not have an inherent physical mechanism for resisting frequency change.

\footnotetext{
${ }^{11}$ The term fast frequency response is also used to mean other things, sometimes as a generic term for fast power injection in response to frequency changes. There is a lack of clear nomenclature standardization.
} 
While inverters do not have stabilizing inertia, their ability to respond quickly can allow them to support grid frequency on what are conventionally considered inertial time scales [15], [2], [11]. This ability to respond quickly can be beneficial in low-inertia power systems such as those in Hawaii because frequency events happen very quickly (i.e. with high ROCOF), so resources providing PFR must do so very quickly. However, there may be increased risks of unintended consequences associated with fast responses [16].

\subsection{Inverter Hardware Characterization}

Five PV inverters and one residential-scale storage inverter were tested to evaluate their frequency-watt control responses in NREL's Energy Systems Integration Facility (ESIF). The PV inverters included three string inverters and two assemblies of microinverters. A controllable AC power supply was used to apply frequency transients to each inverter, and the inverter's response was recorded.

Quantifying the time-domain dynamics of an inverter's response to a frequency change is challenging. One common method of characterizing the time-domain response of a system to a change in an input is to step the input signal from one value to another and record the output response over time. However, many inverters will trip if a step change in frequency is applied; inverters are not designed for this as step changes in frequency do not occur on power systems containing synchronous generation. Even if the inverter does not trip, its response to a step change in frequency may not be indicative of its response to a real frequency event, as the step change may cause its internal frequency measurement to become temporarily inaccurate. Therefore the inverters were tested using relatively fast frequency ramps rather than frequency steps. Frequency ramp rates were in the range one to three Hertz per second for most tests; faster ROCOFs were used in some tests in an attempt to better quantify the inverters'

responses. Test waveforms were recorded at sampling rates between 15 and $50 \mathrm{kHz}$ and postprocessed to calculate power, frequency, and other quantities.

\subsection{PV Inverter Droop Response Characterization}

The responses of the first three inverters were summarized in the previous report [4]. Overall, inverters 1-3 had satisfactory frequency-watt responses considering both response time and steady-state characteristic, especially given that no U.S. utility had yet required frequency-watt control for distributed PV at the time of testing (2016). Inverter 2 was found to have a small overshoot in its power response dynamic as shown in Figure 4, so it was modeled using a second-order transfer function. The remaining inverters were modeled with first-order transfer functions. 

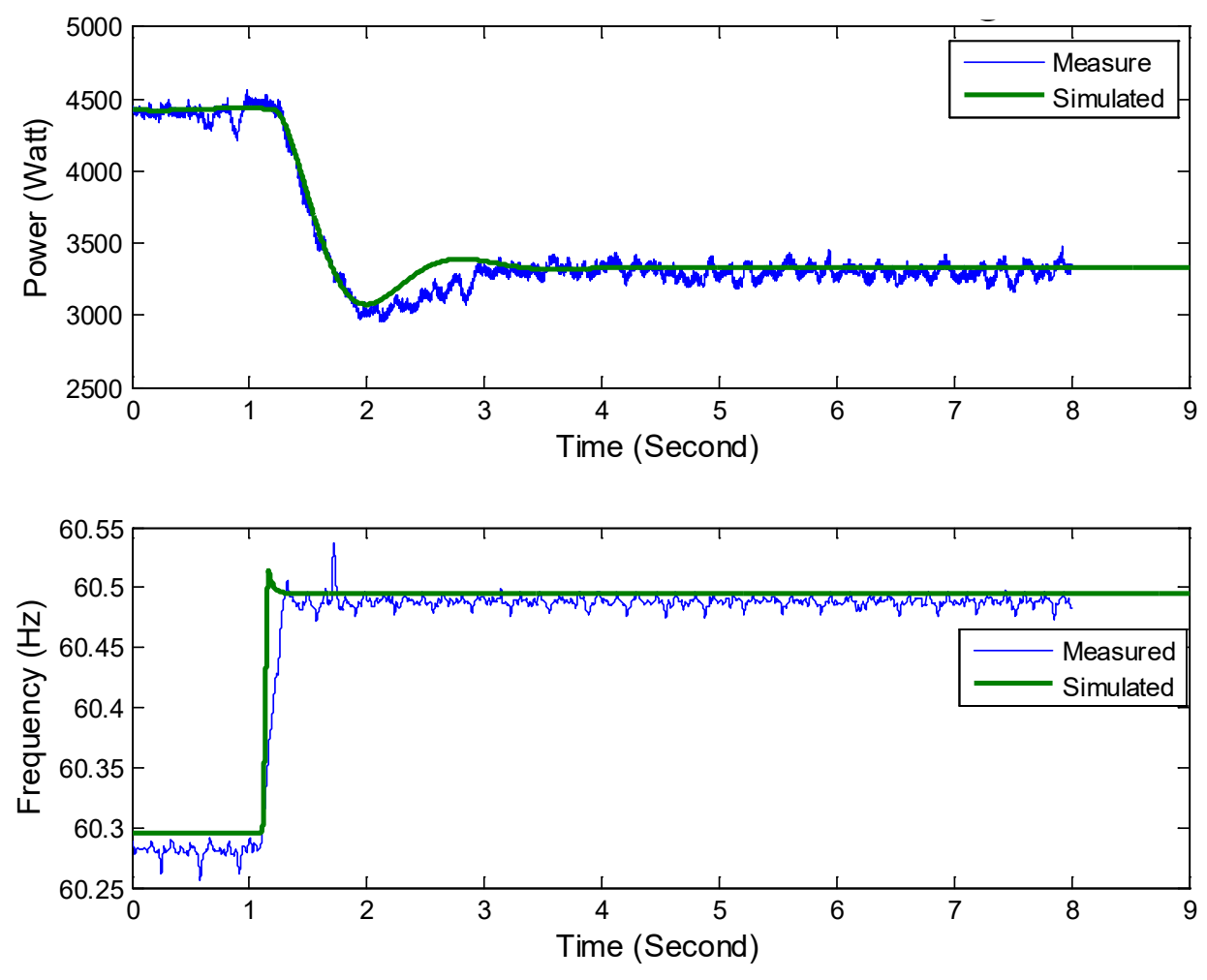

Figure 4: Example of Inverter 2 frequency-watt time response

Inverter 3 was unique in that its frequency-watt response could be programmed to operate below the maximum available PV input power and respond to underfrequency events. At the time of testing (2017), it was the only known residential-scale PV inverter with this capability.

Figure 5 shows the frequency-watt function that can be defined for Inverter 3 . The curve can be programmed asymmetrically as shown in Figure 5, but all tests presented here used symmetrical deadbands and droop slopes. The inverter can participate in frequency regulation for underfrequency events if the pre-disturbance power is smaller than the maximum available 
power, $P_{\max }$, for the inverter. The difference between the pre-disturbance power and maximum available power is defined as reserve power, i.e. $P_{\text {Reserve }}=P_{\max }-P_{\text {pre. }}$.

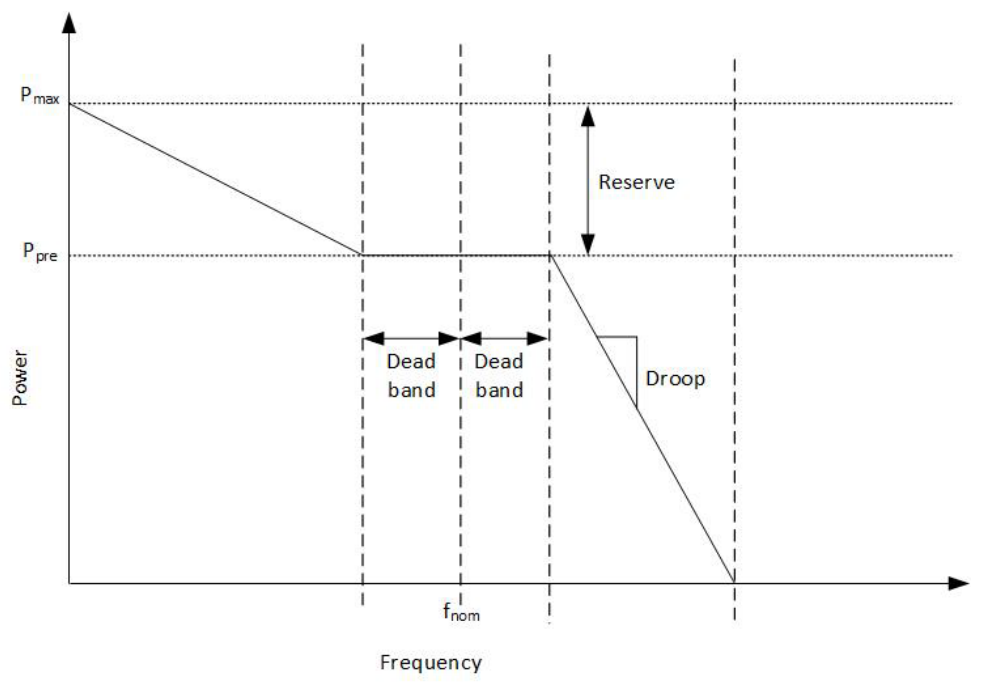

Figure 5: Frequency-watt curve definition for Inverter 3

Figure 6 shows frequency and power data from a frequency-watt test of Inverter 3 with the inverter programmed to operate at $50 \%$ of rated power with $100 \%$ of rated power available (so $50 \%$ of power is reserved). The power-frequency characteristic traced out during the test is shown in Figure 7. The inverter adheres closely to the programmed curve, increasing power for below-nominal frequencies and decreasing power for above-nominal frequencies, as desired.
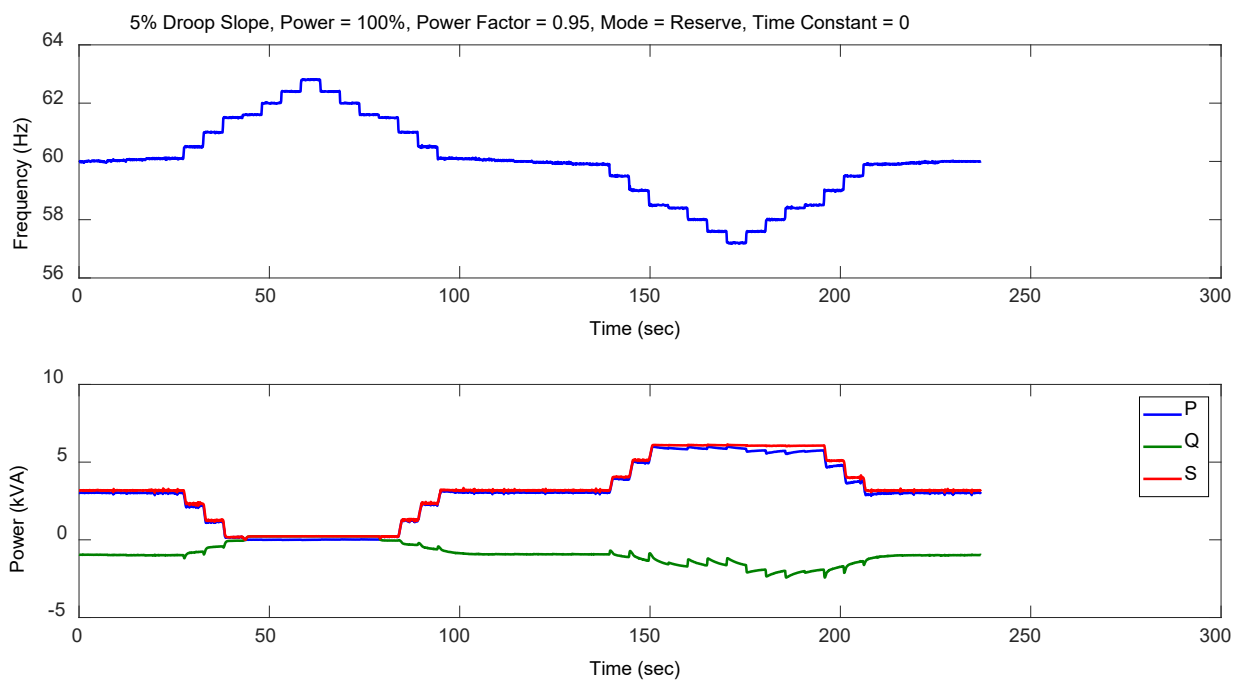

Figure 6: Inverter 3 frequency-watt test with power reserved to allow for underfrequency response 


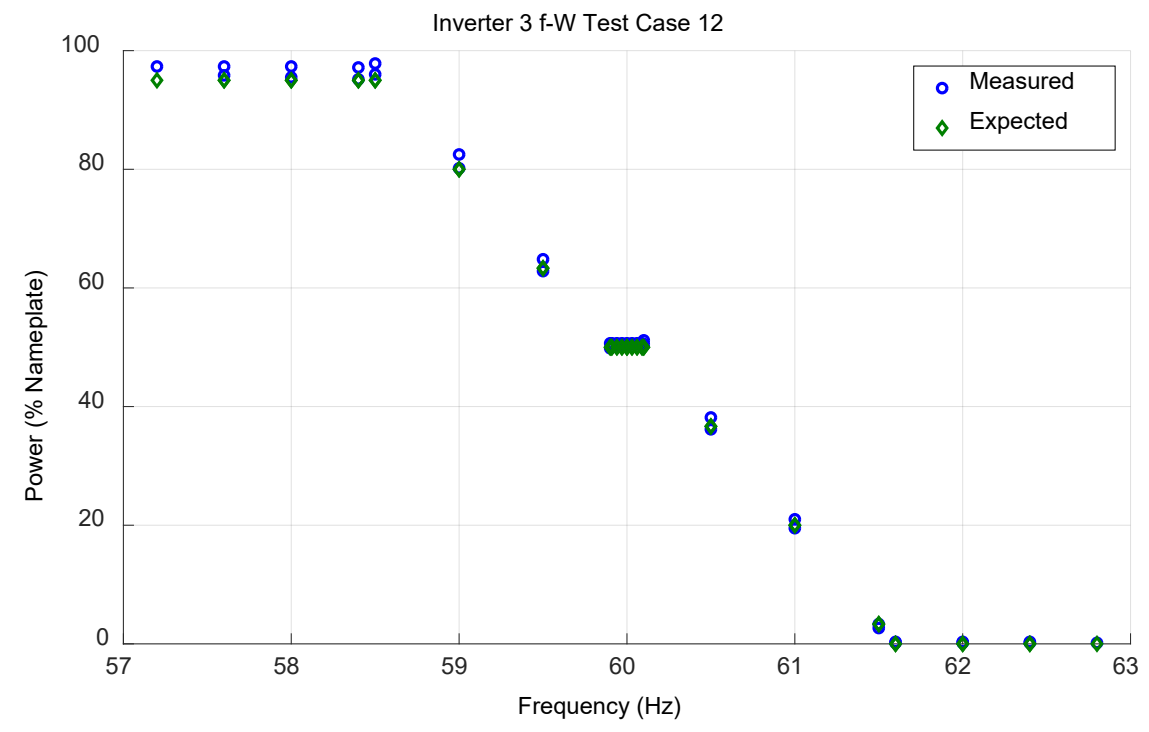

Figure 7: Comparison of Inverter 3 frequency-watt measured characteristic to programmed characteristic

Inverters 4 and 5 were tested after the approval of frequency-watt control and were both certified to UL 1741 SA, as required. This testing occurred under a Hawaiian Electric-funded project related to field testing of advanced inverter functions primarily focused on advanced inverter voltage regulation [17], [18]. The lab testing phase of the pilot offered the opportunity to 
also evaluate frequency-watt control as deployed in the field. Additional lab test results can be found in [19].

Figure 8 shows the time-domain response of Inverter 4 to a change in frequency. The inverter responds within about 0.2 seconds, as desired. The reason for the small oscillations in active power is not known; it may be related to operating away from nominal frequency.
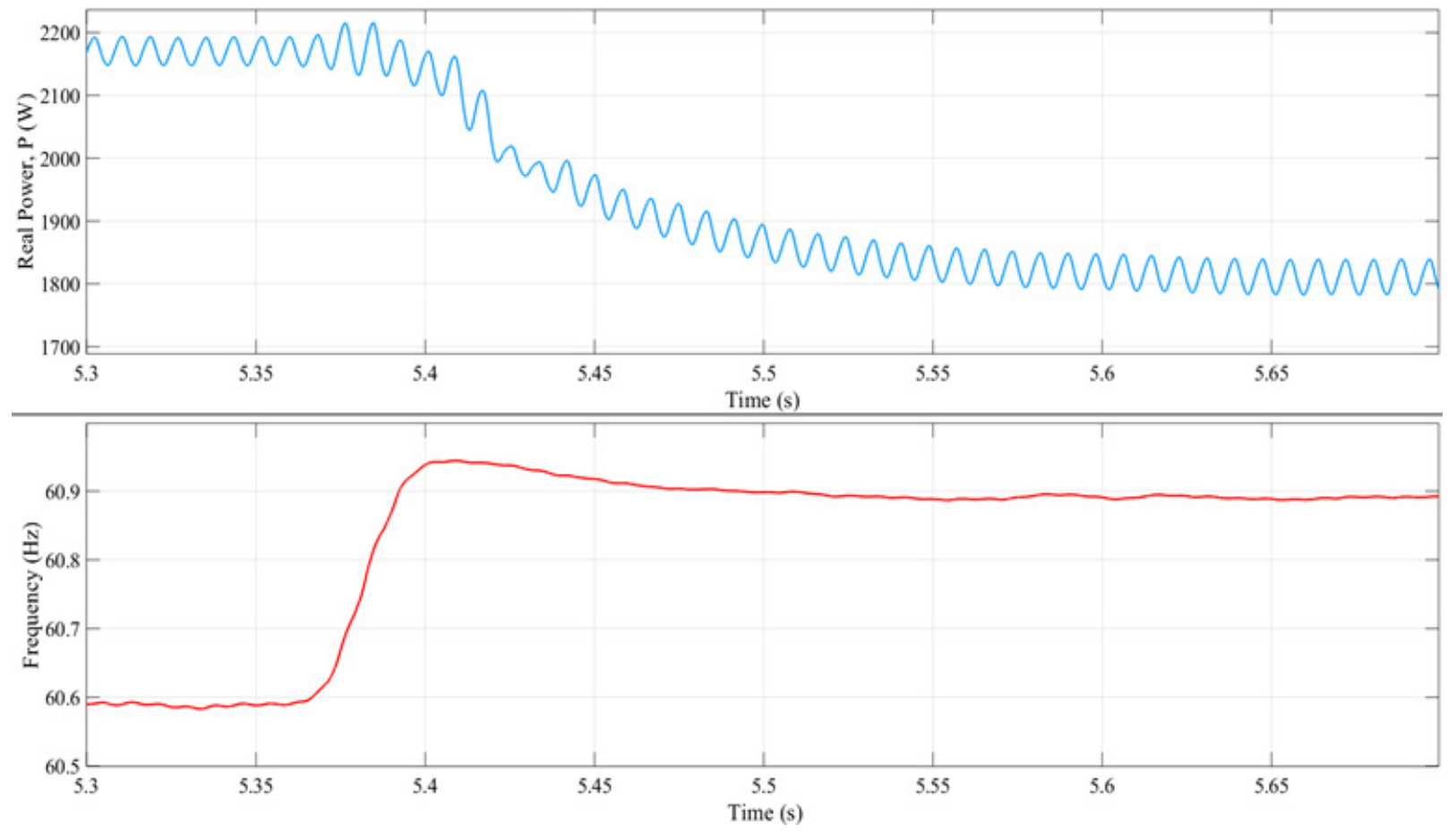

Figure 8: Example of Inverter 4 frequency-watt time response

Figure 9 shows the steady-state frequency-watt characteristic of Inverter 4 as compared to the desired characteristic from SRD V1.1 for three different pre-disturbance active power levels. The inverter appears to be performing frequency-Watt following a specification where the power always reaches zero by a specified frequency, rather than reducing power at a constant slope. This was acceptable on an interim basis per Rule $14 \mathrm{H}$, but should be updated in the future to follow the form of the frequency-Watt function defined in SRD V1.1, which aligns with IEEE 1547-2018. 


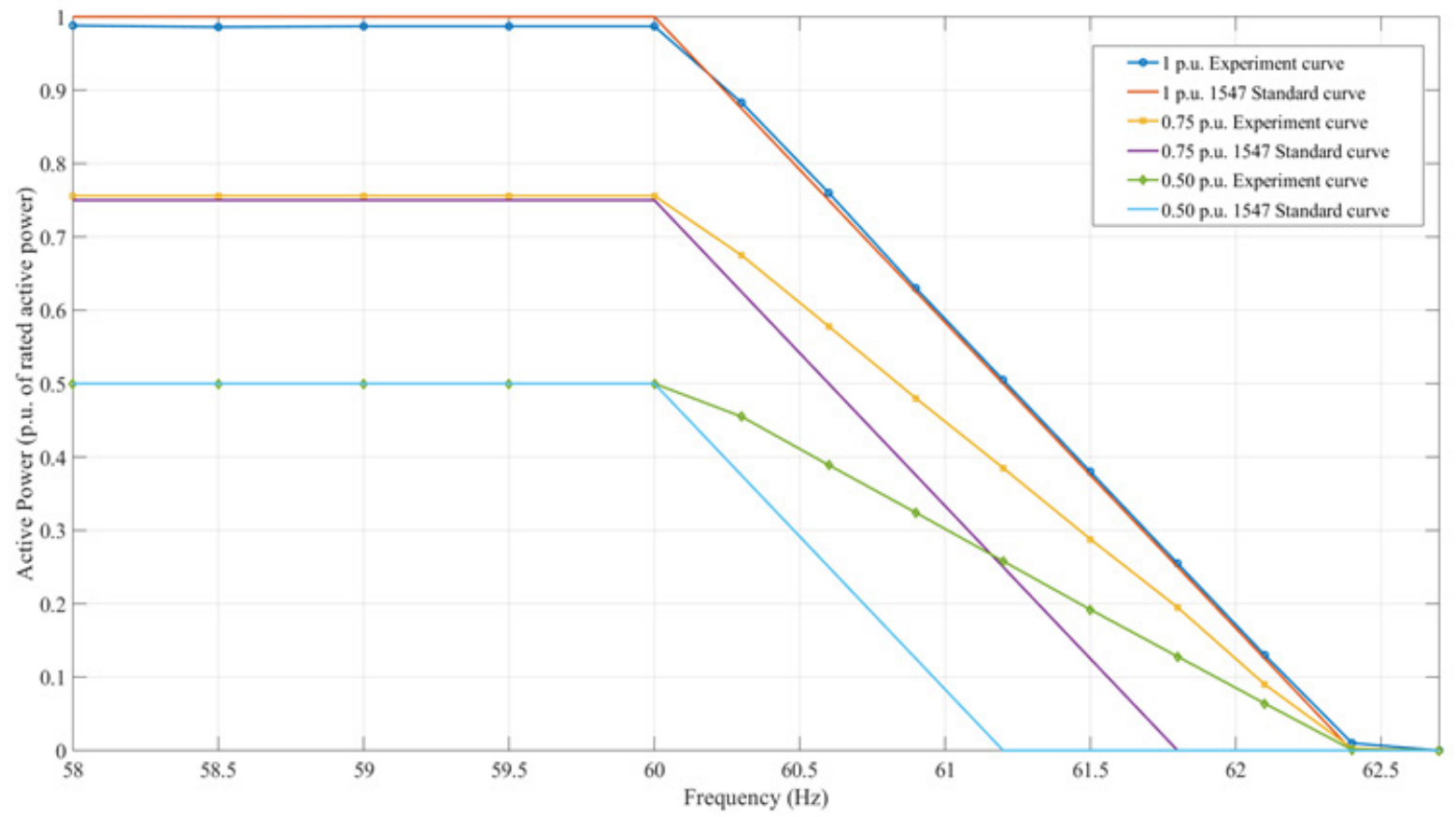

Figure 9: Inverter 4 steady-state frequency-watt response

Figure 10 shows the time-domain response of Inverter 5 to a step change in frequency. Note that the inverter takes much longer to respond than the other inverters tested at about 7 seconds, and it responds in two discrete power adjustments. This inverter would need to respond more quickly to comply with SRD V1.1 and to be effective at mitigating Hawaii frequency events. If deployed widely without updating the response time, this inverter's frequency response could even cause frequency oscillations, at demonstrated in subsequent sections.
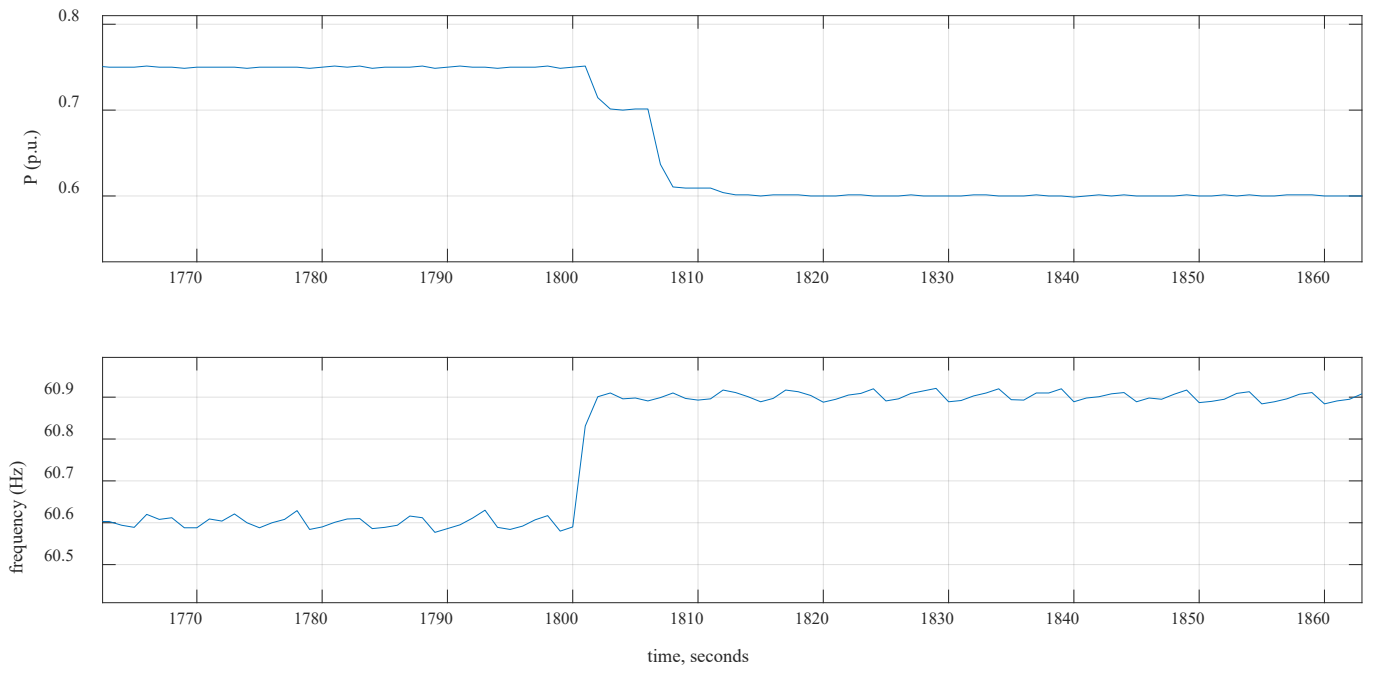

Figure 10: Inverter 5 time domain frequency-watt response

Figure 11 shows the steady-state frequency-watt response characteristic of Inverter 5. Like Inverter 6, it appears to be following a different form of the frequency-watt function from that in 
SRD V1.1 and 1547-2018. This is acceptable on an interim basis per Rule $14 \mathrm{H}$, but should be updated in the future to follow the form of the frequency-Watt function defined in SRD V1.1, which aligns with IEEE 1547-2018.

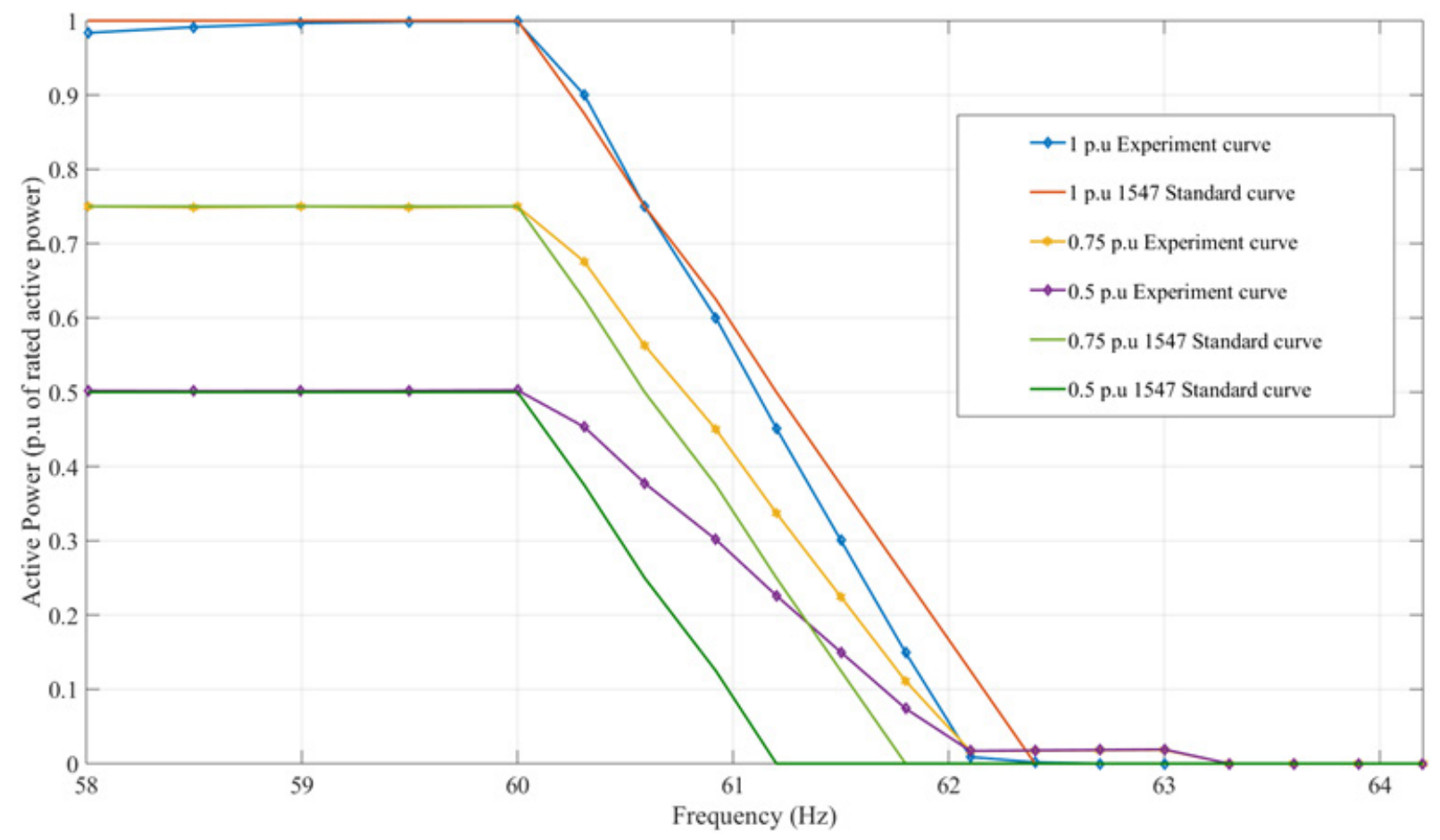

Figure 11: Inverter 5 steady-state frequency-watt response

\subsection{Storage Inverter Droop Response Characterization}

Several manufacturers of residential-scale storage inverters were contacted in 2017 to determine whether they could provide frequency droop response. The majority reported they could not (perhaps not surprisingly given the immaturity of the market at that time). Two inverter manufacturers reported that they could provide droop response, but one of those was not able to support testing at that time. The other manufacturer's residential-scale storage inverter was tested in ESIF to characterize its frequency droop response. This inverter is designated "Inverter 6".

The manufacturer of Inverter 6 reported that while in charging mode (absorbing active power from the grid), the inverter would not perform frequency-watt control. Tests confirmed that the inverter showed no response to frequency changes during charging mode, as depicted in Figure 12: the active power remains constant despite significant frequency changes. The lack of response is not surprising given that, at the time of testing, there was no requirement for frequency-watt response while in charging mode. (As IEEE 1547-2018 is adopted, it will require frequency droop response in both charging and discharging modes.) 

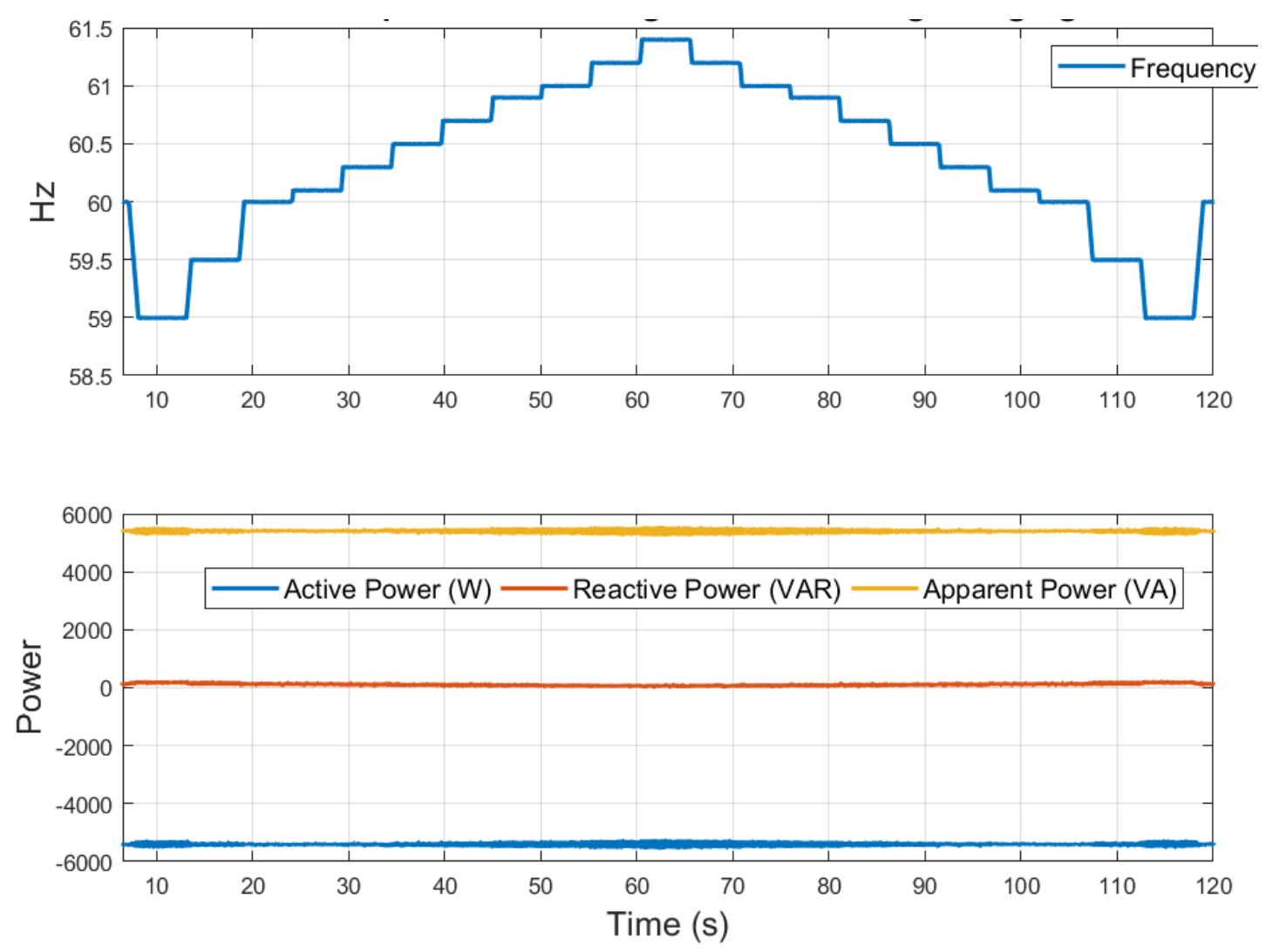

Figure 12: Frequency-watt response of Inverter 6 (storage inverter) while charging

In discharging mode (i.e. exporting power to the grid) Inverter 6 was found to be capable of droop response, as shown in Figure 13. The inverter follows the programmed droop curve accurately. However, the inverter does not increase its rate of discharge for underfrequency events - it only responds to overfrequency, and only in discharging mode.

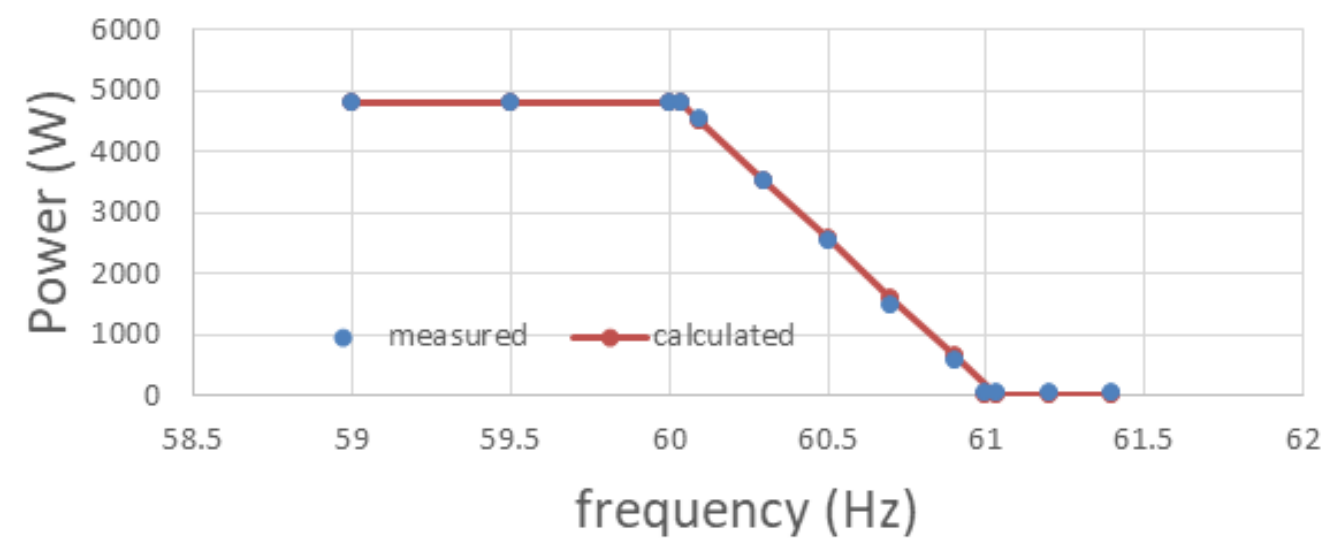

Figure 13: Steady-state frequency-watt response of Inverter 6 (storage inverter) while discharging 
Interestingly, Inverter 6 exhibited a step-wise time-domain response for active power modulation in response to frequency changes. For each frequency change, the inverter holds its power constant for about for 1 second before changing its output power to a level corresponding to the changed frequency; it appears that the frequency-watt response may be implemented using a lookup table that is referenced once per second. The variable time delay response of Inverter 6 was taken into consideration while modeling the inverter response. It was assumed that the timing of power updates will vary between inverters so that for an aggregation of many inverters, the step changes in output power will look like a continuous change. Even if this assumption is not correct, this inverter model makes up a moderate proportion of total DERs, so the step changes are not expected to affect bulk system frequency dynamics. However, the fixed 1second delay could cause instability if many megawatts of this type of inverter were installed. This type of response does not comply with the time response requirements of IEEE 1547-2018, so is expected to be revised in future inverter models. This inverter's power response was modeled using a first-order time constant $\tau$ and a fixed delay $\tau_{\text {delay }}$, as shown in equation (1).

$$
H=\frac{e^{-\tau_{\text {delay }} s}}{\tau S+1}
$$

Figure 14 shows an example of the steady-state droop response of Inverter 6 along with the simulated model response for comparison. Figure 15 compares the simulated aggregate inverter response to the response of a single hardware inverter when both are exposed to a simulated overfrequency event with damped oscillations.
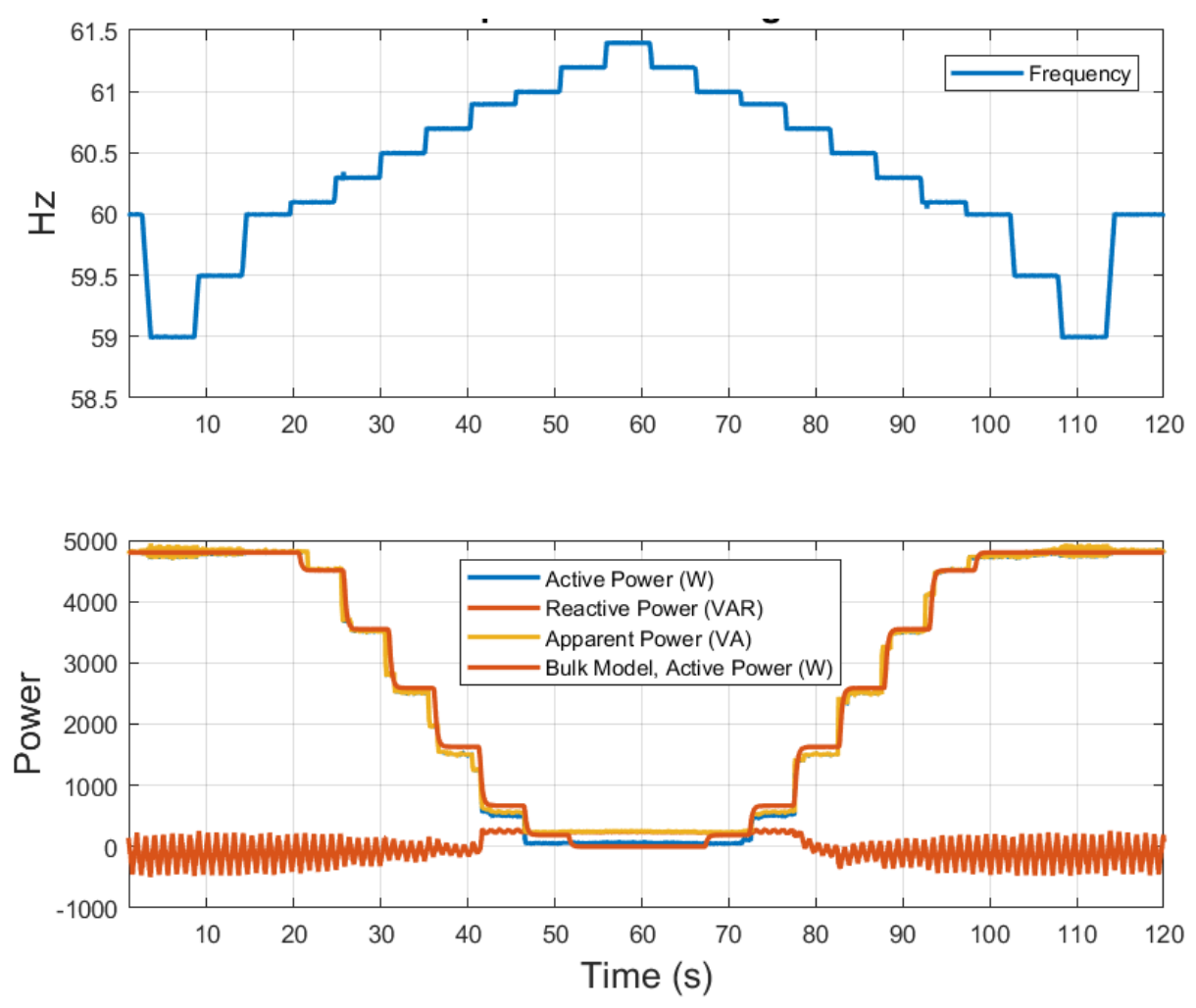

Figure 14: F-W response of StorEdge inverter during discharging 

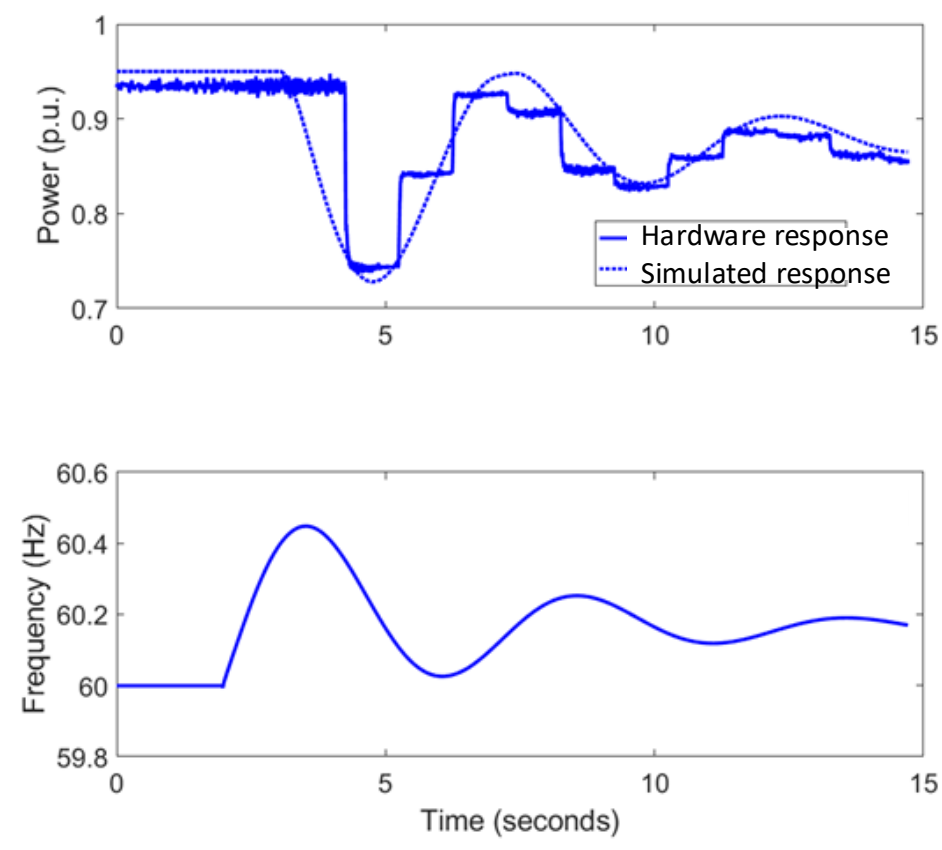

Figure 15: Time domain droop response of Inverter 6 while discharging, compared to simulation of aggregate response of multiple inverters of this type.

In summary:

- Four out of the six inverters tested met the time response requirements needed to assist with frequency stability in Hawaii. The remaining two had slower responses with fixed delays, which could lead to instability if deployed widely.

- Tests of inverters sold since the mandatory activation of frequency-watt control show that manufacturers have not yet implemented the form of the frequency-watt function specified by Hawaiian Electric and IEEE 1547-2018.

- All of the inverters tested were able to provide overfrequency response. Only one was able to provide underfrequency response.

\subsection{Stability Analysis of Inverter-Machine Systems}

To supplement the simulations and PHIL experiments presented in Sections 4.0 - 5.0, this section presents a theoretical analysis used to study the interactions in a mixed-source power system. The small-signal dynamic stability analysis summarized here was presented in greater detail in [20]. To enable the small-signal analysis, a simplified aggregated reduced-order model of the Oahu power system was developed. The model was linearized around a steady-state operating point and the eigenvalues of the linearized model were obtained following the approach presented in [21]. The key modes relevant to the frequency dynamics were identified using participation factors, and eigenvalue trajectories were plotted for variations of different parameters.

Note that the simple models used here are intended specifically to examine interactions between frequency-watt controlled inverters and conventional generators. The models used 
here are not intended to examine potential interactions at other time scales, such as between inverter current controllers and synchronous machines. In addition, these models do not include certain dynamics that should be examined separately, such as turbine shaft modes and interarea modes.

\subsection{Reduced-order Aggregate System Model}

A simplified reduced-order aggregated two-source model of the power system was developed for this study, shown in Figure 16. All the synchronous generator units were aggregated into a single source and the inverter units were aggregated into another unit, with interconnecting impedance between sources. This is similar to the aggregation approach used in [22] to analyze the impact of penetration level of grid-following versus grid-forming sources in a microgrid.

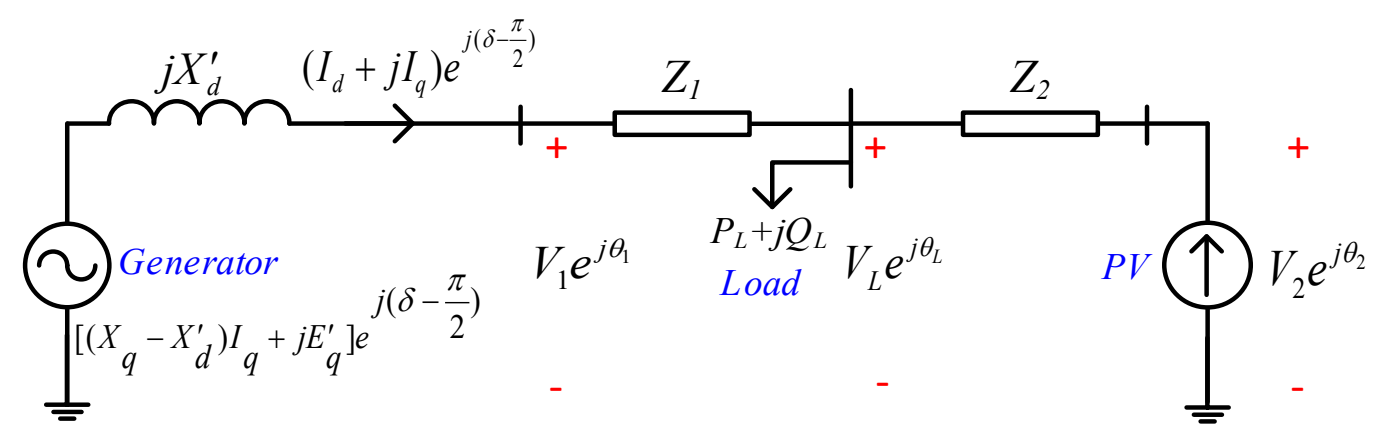

Figure 16: One-line diagram of reduced-order network with aggregated sources.

\subsection{Conventional Generator Aggregation}

A system identification approach was used to aggregate all conventional generator into an approximately equivalent single generator model. A governor-only model of all the synchronous generators in Oahu that closely matches field data was earlier developed [4]. This model includes the governors, turbines, and droop response for each unit in the system. To further simplify this model into a single equivalent generator, the highest order transfer functions for the governor $G(s)$ and turbine $T(s)$ among all units were used to construct an equivalent aggregated generator. The electrical part of the aggregated synchronous generator is modeled as a standard two-axis synchronous machine turbine-governor model (ignoring stator dynamics) and an IEEE type-I exciter, as shown in Figure 17 . The values for parameters for the exciter model are taken from [21]. The aggregate inertia was obtained by adding the inertia constants of all units. For the same disturbance input (load or generation change), the output response (frequency) must match to achieve equivalence between the aggregated model and the original model. System identification tools in Matlab were used to tune the coefficients of $G(s)$ and $T(s)$ to obtain similar responses for the same input disturbance $( \pm 10 \%$ net load change). A close match of the frequency output was obtained after parameter estimation, as shown in Figure 18. Table 2 shows the estimated parameters, as used here. 


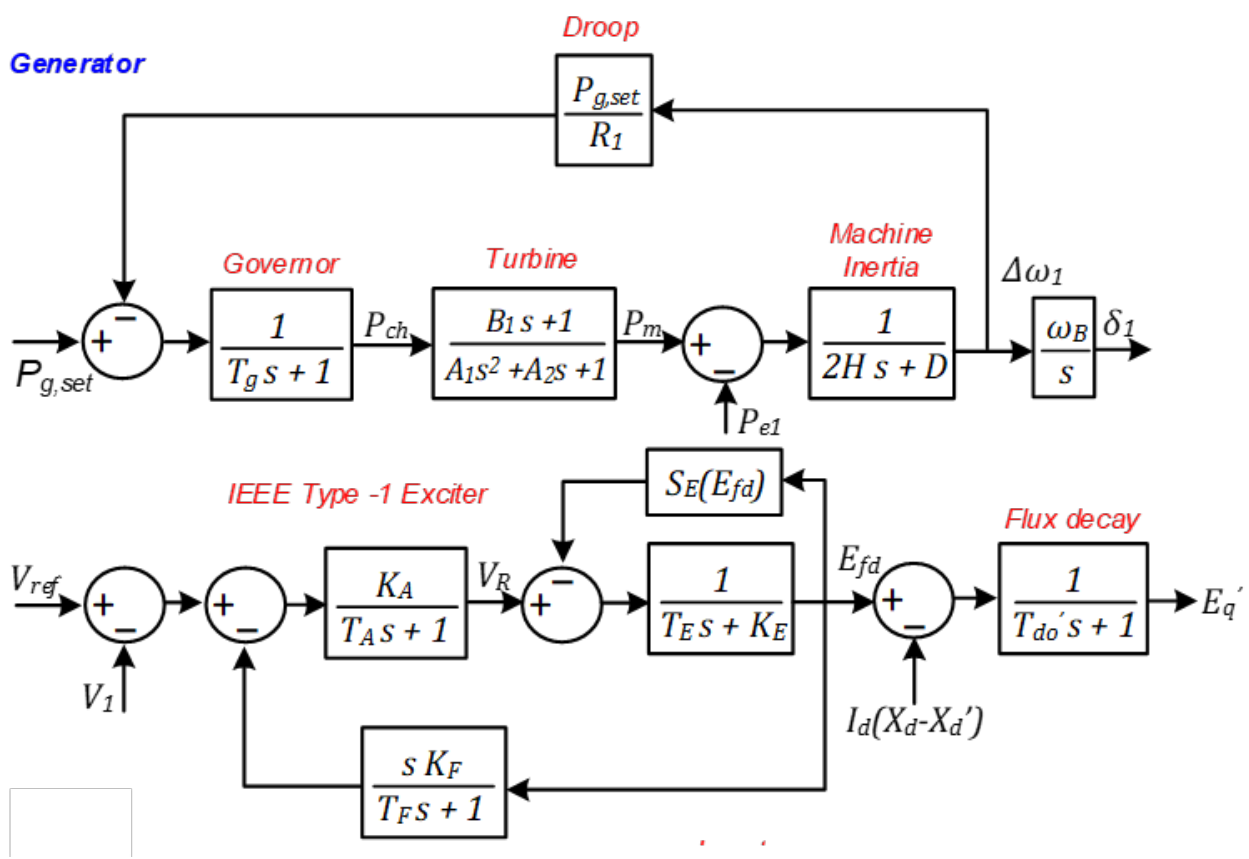

Figure 17: Aggregate conventional generator model used for small-signal stability analysis.

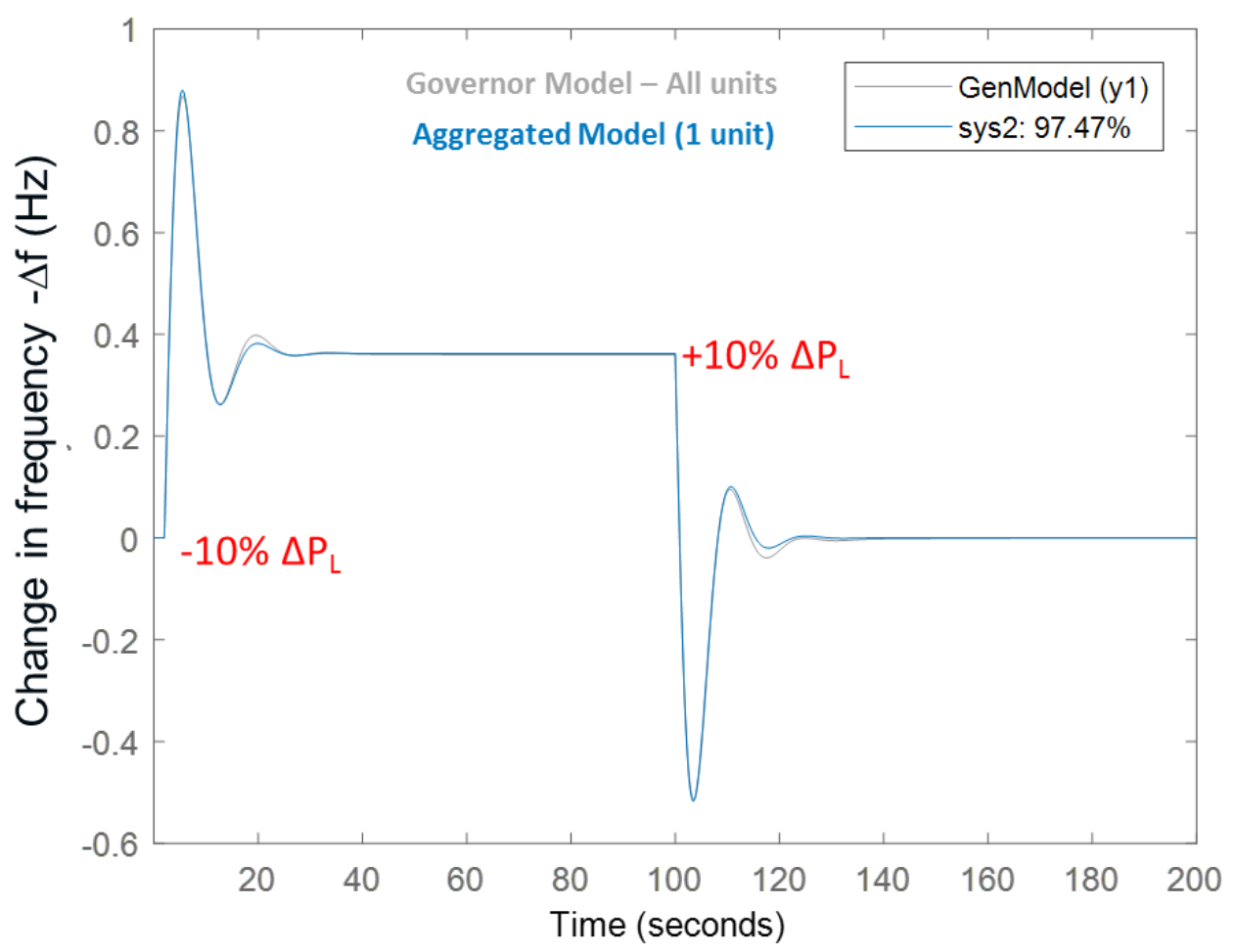

Figure 18: Comparison of frequency responses of the aggregated generator model and the full governor-only model. 
Table 2: Single-machine Aggregate Model Parameters.

\begin{tabular}{|l|l|}
\hline Parameter & Value \\
\hline$H(s)$ & 3.5 \\
\hline$D$ & 0.02 \\
\hline$T_{g}(s)$ & 0.2 \\
\hline$B_{1}$ & 2.136 \\
\hline$A_{1}$ & 4.42 \\
\hline$A_{2}$ & 7.58 \\
\hline$R_{1}$ & 0.0589 \\
\hline
\end{tabular}

\subsection{Inverter Aggregation}

Inverter-based generators behave as power-controlled current sources. The current controller dynamics of inverter-based generators are much faster than the power regulation dynamics and were thus not modeled. The power controllers were assumed to have a similar structure with similar parameters and are thus aggregated into a single unit. Testing in Section 2 and in [4] suggests the power controller response typically can be approximated with a first-order transfer function (time constant $T_{3}$ in Figure 19). The frequency measurement (phase-locked loop, PLL) is also modeled as a first order system with a time constant $T_{1}$. The nonlinearities in the frequency-watt function such as dead-band and power limits are ignored for the small-signal study.

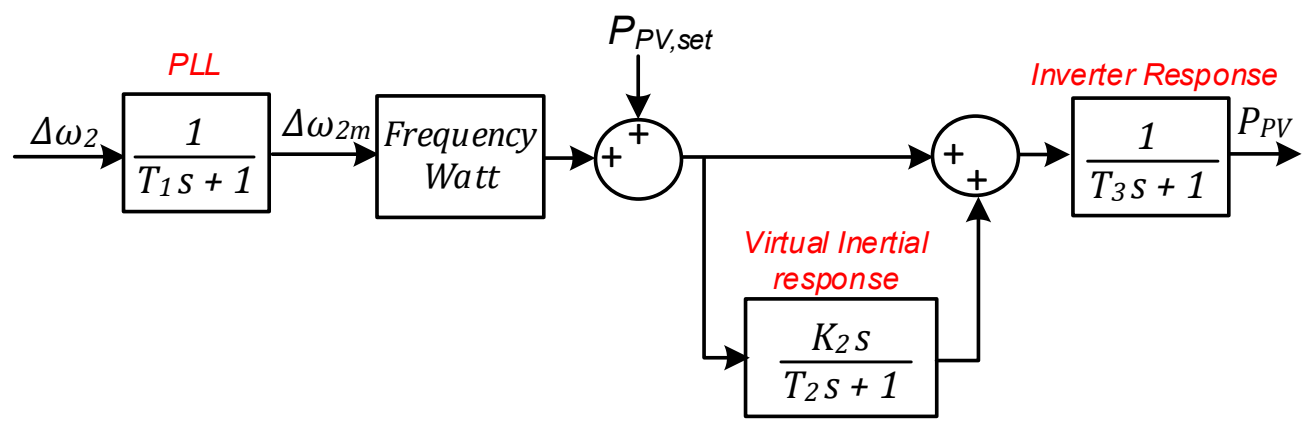

Figure 19: One-line diagram of reduced-order network with aggregated sources.

The aggregate inverter-based generation model was also configured to allow for study of virtual inertial response. In this case, a component of power proportional to the ROCOF was used in addition to the droop response. This is similar to the acceleration torque response from rotating machines but with inherent frequency measurement and controller time delays. The virtual inertia controller can be disabled by setting its gain, $K_{2}$, to zero.

\subsection{System Model}

The electrical network was modeled as a three-bus system with interconnecting impedances between the sources and load, as seen in Figure 16. Constant power loads were situated 
between the two sources. The stator and network equations were treated as algebraic constraints, and the system was linearized around the steady-state operating point. The eigenvalues are then computed from the linearized state-space matrix.

Because this study is aimed at understanding the interactions in a mixed-source system from first principles, and the focus is on investigating the impact of inverter controls such as frequency-watt on the dynamic response, the use of a simplistic model is necessary. The aggregated model is not an accurate representation of the electrical network to capture all critical modes. The distribution system with PV and other DERs interconnected at many nodes is a complicated heterogenous network and interactions between multiple generators/areas and many other dynamic interactions are not accounted for. Aggregating such a large complicated network itself is a relatively unexplored research topic and could be a future research effort.

\subsection{Small-signal Stability Analysis}

The simplified aggregate system described above was linearized around the steady-state operating point. The steady-state operating point was obtained by solving the power flow equations. Eigenvalues were calculated for varying inverter-based generation penetration levels with varying system parameter values. The instantaneous inverter penetration level was defined as the percentage of total load power supplied by inverter-based generation. Variations in system inertia $H$, inverter response time constant $T_{3}$, inverter droop $R_{2}$, and inertial response gain $K_{2}$ were studied using their respective eigenvalue trajectories, as shown in Figure 20 Figure 23. Constant damping factor $(\zeta)$ lines are shown in each figure to visually aid in analyzing the eigenvalue trajectory plots (dashed lines in steps of 0.1 , with top line indicating $\zeta=0.1$ and $x-$ axis corresponding to $\zeta=1$ ). Time domain responses are plotted for a step decrease of $5 \%$ in total load in the system to validate the eigenvalue analysis. The parameters used for the baseline case are given in Table 3 and Table 4 . The given parameters are specified per unit on the system base of 1000 MVA and $46 \mathrm{kV}$.

Participation factor calculations revealed two slow oscillatory modes that have the maximum impact on the frequency output response and were thus the deemed critical. The axes of plots were scaled to clearly depict these critical modes.

Table 3: Baseline Generator Parameters for Small Signal Stability Study

\begin{tabular}{|c|c|c|c|c|c|}
\hline Parameter & Value & Parameter & Value & Parameter & Value \\
\hline $\mathrm{H}(\mathrm{s})$ & 3.5 & $\mathrm{~K}_{\mathrm{A}}$ & 20 & $\mathrm{X}_{\mathrm{d}}$ & 0.8979 \\
\hline $\mathrm{D}$ & 0.02 & $\mathrm{~T}_{\mathrm{A}}(\mathrm{s})$ & 0.2 & $\mathrm{X}_{\mathrm{d}}$ & 0.2995 \\
\hline $\mathrm{T}_{\mathrm{g}}(\mathrm{s})$ & 0.2 & $\mathrm{~K}_{\mathrm{E}}$ & 1 & $\mathrm{X}_{\mathrm{q}}$ & 0.646 \\
\hline $\mathrm{B}_{1}$ & 2.136 & $\mathrm{~T}_{\mathrm{E}}(\mathrm{s})$ & 0.19 & $\mathrm{X}_{\mathrm{q}}{ }^{\prime}$ & 0.646 \\
\hline $\mathrm{A}_{1}$ & 4.42 & $\mathrm{~K}_{\mathrm{F}}$ & 0.0012 & $\mathrm{~T}_{\mathrm{do}}{ }^{\prime}(\mathrm{s})$ & 7.4 \\
\hline $\mathrm{A}_{2}$ & 7.58 & $\mathrm{~T}_{\mathrm{F}}(\mathrm{s})$ & 1 & $\mathrm{R}_{1}$ & 0.05 \\
\hline
\end{tabular}


Table 4: Baseline Generator Parameters for Small Signal Stability Study

\begin{tabular}{|c|c|}
\hline Parameter & Value \\
\hline$T_{1}$ & 0.05 \\
\hline$T_{2}$ & 0.25 \\
\hline$T_{3}$ & 0.5 \\
\hline$K_{2}$ & 0 \\
\hline$R_{2}$ & 0.05 \\
\hline$Z_{1}$ & $0.01+0.05 j$ \\
\hline$Z_{2}$ & $0.01+0.05 j$ \\
\hline
\end{tabular}

\subsubsection{Variation in Inverter Penetration Level}

Eigenvalue trajectories are plotted for variation in parameters at specific penetration levels between $50 \%$ and $90 \%$ in Figure 20 - Figure 23. Each penetration level is indicated by a color and labeled in that color. In this simple model, except where otherwise indicated, variations in inverter penetration are not accompanied by variations in system inertia, as they would be in a real system. Instead, variations in inertia are studied separately below. In general, increasing penetration levels result in a shift in eigenvalues to the right (lower real part, $|\sigma|)$ and higher oscillation frequency, $|\omega|$. The response can be expected to be slower and more oscillatory (less damped) at higher penetration levels.

\subsubsection{Variation in Mechanical Inertia $(H)$}

The aggregate system mechanical inertia constant was varied from 1.5 to 6 seconds; the eigenvalue trajectories are plotted on the left in Figure 20. The increase in inertia clearly results in a slower and more damped response (higher $|\sigma|$ and lower $|\omega|)$. The damping factor increases with increase in inertia and the oscillation frequency decreases. With higher penetration levels ( $90 \%)$, a low inertia might result in an unstable system. (In practice, other factors not modeled here may limit penetration to lower levels unless non-conventional controls are implemented.) The time domain responses for a $5 \%$ load change with inertia values of 1.5 , 3, 4.5, and $6 \mathrm{~s}$ are also shown in Figure 20 at $70 \%$ instantaneous inverter penetration. Lower inertia results in a more oscillatory response with a higher apex. Thus, higher inertia improves frequency dynamics and aids system stability. 

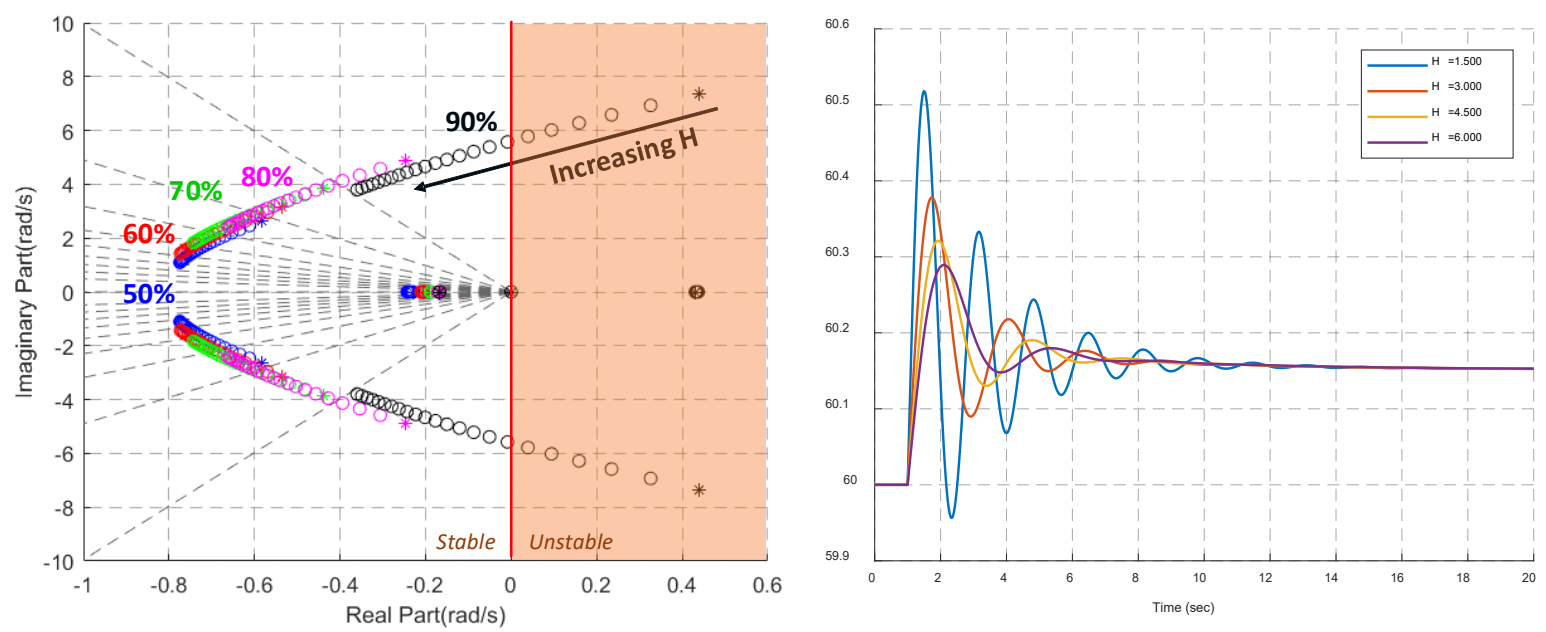

Figure 20: Eigenvalue trajectories (left) and time domain responses (right) for variation in system inertia $(H)$ from 1.5 to $6 \mathrm{~s}$.

Even though system inertia is not an easily-controllable parameter, these plots offer an understanding of the relative performance with inertia variation. In small power systems like Oahu, increase in penetration levels of PV and other inverter-based generation is often accompanied by the displacement of synchronous generators and consequent reduction in system inertia. Synchronous condensers can contribute somewhat to system inertia. Grid forming sources may also be used to improve the stability of the system at such penetration levels.

\subsubsection{Variation in DER Droop Slope $\left(R_{2}\right)$}

The droop parameter of the aggregated inverter unit was varied from $2 \%$ to $8 \%$. Increases in $R_{2}$ (less aggressive droop) provide a better damped response (higher $|\sigma|$ and lower $|\omega|$, as seen in Figure 21. The damping factor increases with increase in $R_{2}$. Lower (more aggressive) droop may cause instabilities at higher penetration levels. A higher value of droop implies a lesser participation of inverter-based generation in the frequency response. This results in a larger steady-state frequency difference with higher droops and hence a higher frequency apex. In the right portion of Figure 21, the time domain responses for a 5\% load change for different droop values at the $70 \%$ penetration level verify the observations made from the eigenvalue analysis. A higher value of droop results in a well damped response but a lower droop increases the participation of $\mathrm{PV}$ in the frequency response thus reducing the peak frequency deviation. $\mathrm{A}$ design tradeoff between damping (stability) and frequency apex must be made to choose the DER droop slope. 

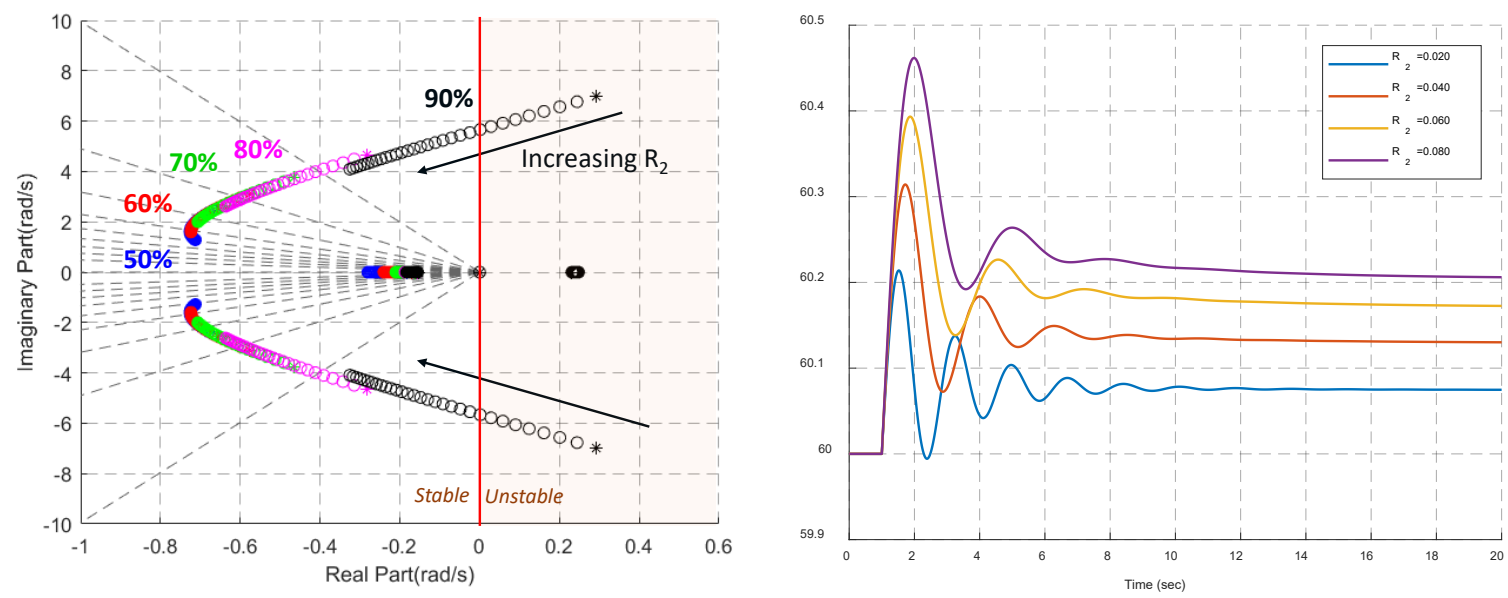

Figure 21: Eigenvalue trajectories (left) and time domain responses (right) for variation in in DER droop $\left(R_{2}\right)$ from $2 \%$ to $8 \%$.

\subsubsection{Variation in Inverter Response Time Constant $\left(T_{3}\right)$}

The inverter power control time constant $T_{3}$ was varied from $0.1 \mathrm{~s}$ to $3 \mathrm{~s}$. The eigenvalue trajectories obtained at different penetration levels are shown on the left in Figure 22. An increase in $T_{3}$ results in a slower response (lower $\left.|\sigma|\right)$; the damping factor decreases as the eigenvalues move right causing a poorly damped and slower response. The inverter time constant has a significant impact on the response as a wider variation in the real part $|\sigma|$ is observed compared variations due to other parameters. Time domain responses for a $5 \%$ load increase (Fig. 9b) verify the observations presented. Increasing $T_{3}$ also results in a higher frequency peak, as shown in on the right in Figure 22.
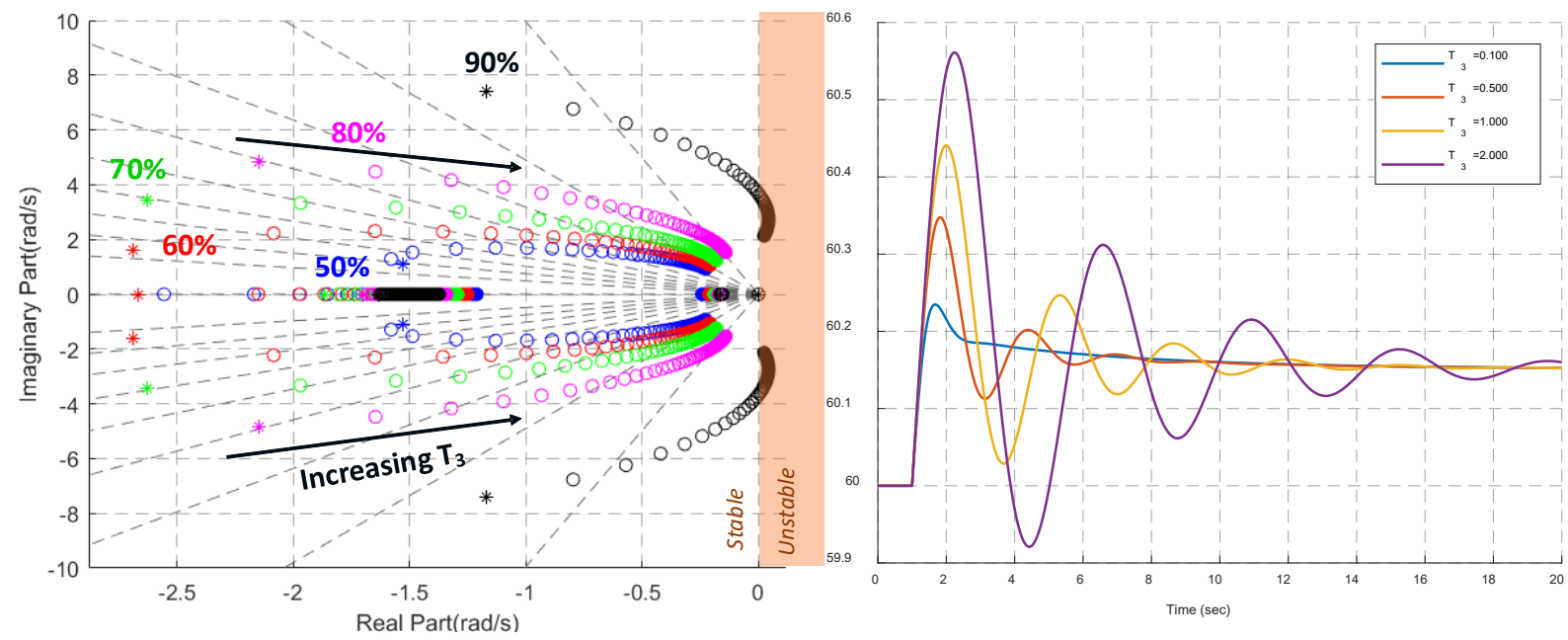

Figure 22: Eigenvalue trajectories (left) and time domain responses (right) for variation in inverter response time constant $\left(T_{3}\right)$ from 0.3 to $3 \mathrm{sec}$.

The small signal study suggests that a smaller time constant (faster inverter response) is better for the system frequency dynamics, and slower time constants negatively affect the response. It must be noted that other interactions such as sub-synchronous resonance/control interactions, torsional modes, etc., are not modeled in the small signal analysis. 


\subsubsection{Variation in Virtual Inertial Gain $\left(K_{2}\right)$}

The virtual inertial response gain $\left(K_{2}\right)$ is varied from 0 to 2 at different penetration levels; eigenvalue trajectories are shown in Figure 23 at left. The control modification adds another slower eigenvalue that affects the frequency. Initially, for small values of $K_{2}$, an increase in the inertial gain seems to improve the damping of the electromechanical modes and enables a faster response. With further increase in $K_{2}$, the response becomes more oscillatory and slower. At higher penetration levels, the stability of the system is sensitive to $K_{2}$, eventually becoming unstable. The time domain responses on the right in Figure 23 help obtain a better picture (again shown at $70 \%$ penetration). An initial increase in gain improves the response significantly (up to $K_{2} \sim 1$ ); the peak is much lower with inertial gain and the response is well damped. With further increase, the response is slowed down as observed from the outer envelope, with undamped oscillations in the inner envelope. Clearly, an increase in $K_{2}$ reduces the peak for all values of gains, but slows down the response at very high values. Hence, there is a tradeoff between speed of response, damping/stability, and peak frequency in the design of virtual inertial response.
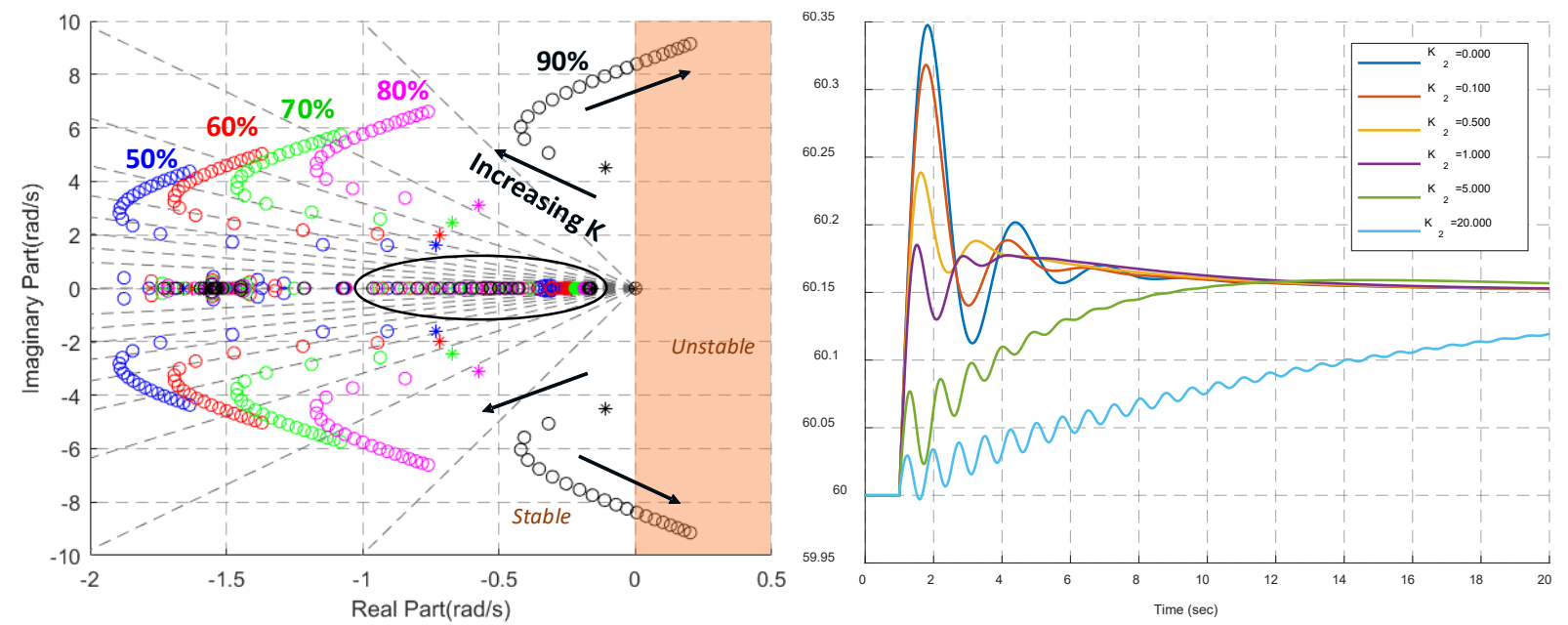

Figure 23: One-line diagram of reduced-order network with aggregated sources.

\subsection{Comparison with Bulk System Simulation Results}

The results from this reduced order aggregated model study qualitatively agree with the PSS/E results presented in the next section and in [4]; recommendations regarding inverter response time, droop slope and inertial response are similar. The approach used here provides fundamental understanding of the key dynamics at play and makes it easier to gain design insights and test controller modifications than a bulk system study.

\subsection{Bulk Power System Simulations}

This section describes simulations conducted to examine the effects of PV inverter frequencywatt control on the transient stability of the Oahu bulk power system. Hawaiian Electric's dynamic model of Oahu's bulk power system in the year 2019 (as projected in 2016) was used for these simulations. Siemens' PSS/E software was used, and a user-defined model (UDM) of 
aggregate distributed PV generation was developed and tuned based on lab testing of residential-scale PV inverters.

For overfrequency events, the specific generation dispatch modeled was a lightly loaded case with high non-synchronous generation (the "light spring" mix), as this represents the highest stability risk for loss-of-load scenarios on Oahu. The 2019 light spring PSS/E model and the PV UDM were used to simulate a variety of loss-of-load cases. Those simulation results were described in the interim project report [4] and in an IEEE paper [23].

\subsection{Distributed Inverter-based Generation User-defined Model for PSS/E}

Current-controlled PV inverter design has been extensively described in the literature; for example, see [24] and the references therein. Typically, a dq-frame current-controlled inverter controller consists of an inner current control loop and an outer power control loop. The dqframe is synchronized with the grid voltage using a phase locked loop (PLL). The current control loop is responsible for regulating the output current of the inverter, while the power control loop is responsible for adjusting the reference current for the current controller in order to control the output power of the inverter. Typically, a PV inverter is controlled to produce the maximum available power based on a maximum power point tracking (MPPT) controller, and a battery inverter is controlled to follow a power reference command generated either from local information or from an external controller. This report does not consider grid forming inverters, which follow a different control scheme.

Figure 24 shows the simple model used here to capture distributed PV inverter frequency-watt dynamics. For bulk power system transient stability analysis, the fast switching transients of inverters occur on a much faster time scale than the power system dynamics and can be neglected. Hence inverters are typically represented as controlled power sources. In this report, the inverter is modeled by a first order transfer function with a time constant which models the speed of response of the inverter's power control. The frequency-watt function is modeled as a lookup table indexed by the measured frequency of the system. An optional first order block is used to model the frequency measurement dynamics, i.e. the PLL. In the positive sequence modeling software (PSS/E) used here does not model the actual voltage waveform, so the PLL cannot be represented directly. In Figure 24, $T_{1}$ represents the time constant associated with frequency measurements, which roughly accounts for PLL and other related dynamics; $T_{2}$ represents the inverter's power regulation response time; $P_{\text {set }}$ represents the power set point of the inverter in steady state; and $P_{\text {out }}$ is the total output power of the inverter. The frequency-watt function is modeled using droop and deadband values as shown in Figure 25. Other equivalent parametrized representations could be used as well [23]. For typical PV inverter operation, the inverter is usually exporting its maximum available power, so $P_{\text {set }}$ is equal to the maximum available PV power, $P_{\text {avail. }}$ Thus a typical PV inverter only responds to overfrequency events and cannot increase power in response to underfrequency events. It is possible for PV inverters to operate with power reserve [2], [11], [15], allowing them to increase power in response to underfrequency events, and it is of course also generally possible for storage inverters to increase power in response to underfrequency events. For the purposes of this report, the DCside dynamics of the battery or PV array powering the inverter are assumed to be faster than the system frequency dynamics and are neglected. However, particularly in the case of PV inverters, this assumption should be examined in future work. Once the output power of the inverter is determined by the model, a current source representation is connected to the PSS/E transmission network and used in the transient stability simulations. 


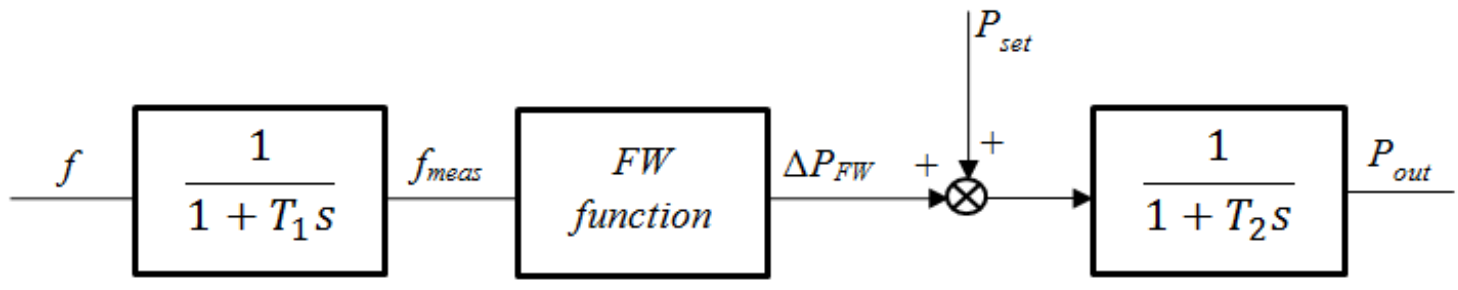

Figure 24: Distributed inverter PV model including frequency-watt function

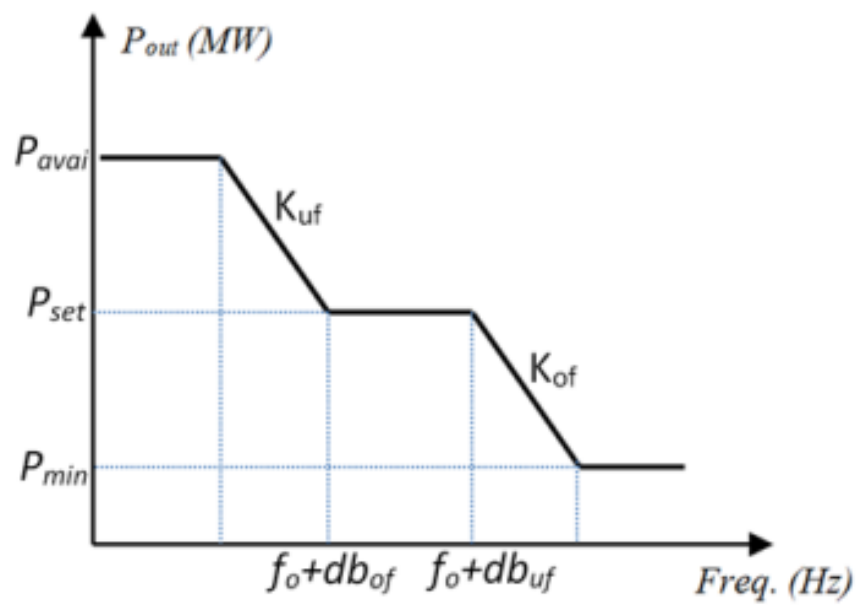

Figure 25: Frequency-watt function parameters

\subsection{DER Inverter Responses to Transmission Faults}

The user-defined model of distributed inverter-based generation was also updated to include an approximate representation of inverter responses to undervoltage events, including momentary cessation behavior, as required in IEEE 1547-2018. Momentary cessation is an inverter control behavior in which the inverter temporarily stops producing current, typically in response to an abnormal grid condition such as a low voltage, and then quickly recovers back to the pre-event current once the abnormal grid condition subsides. Simulations DER inverter responses to transmission faults causing undervoltage events on Oahu can be found in two IEEE papers: [25], [26], and are not repeated here. The primary conclusions were:

- Momentary cessation can be detrimental to bulk system stability, especially in low inertia systems.

- If momentary cessation is used, the recovery process back to normal output should generally be fast to avoid exacerbating the impact of the momentary cessation.

Momentary cessation is currently required by IEEE 1547 for Category III DERs (those with the greatest ability to ride through abnormal grid conditions). This requirement represents a compromise between the desire of distribution planners for DERs not to affect existing protection schemes and the need of transmission planners for generation to remain stable. Momentary cessation is now prohibited (with limited exceptions) for inverter-based resources under the jurisdiction of NERC (i.e. larger transmission-connected resources). It is possible that as Hawaii transitions to very high levels of inverter-based generation, it may be necessary or 
advantageous to transition DERs away from inverter-based generation. This would mean deviating from IEEE 1547 and may require modifications to distribution protection schemes, so would need to be rigorously justified in future work.

\subsection{Simulation of Overfrequency Events}

In [4], various simulations were presented using the user-defined inverter-based generation model with Hawaiian Electric's 2019 light spring PSS/E dispatch scenario, summarized in Table 5. This dispatch scenario was identified by Hawaiian Electric as the worst-case scenario for loss-of-load events due to the smaller quantity of synchronous generation and higher level of inverter-based generation. The goal of the simulations was to determine the sensitivity of the system frequency response to parameters including:

- Inverter's power control response time

- Frequency-watt function droop slope

- Penetration of frequency-watt-enabled inverters.

Those simulations and their results are described in [4]. The primary conclusions were:

- The system response is highly sensitive to inverter power response time, with faster response times generally giving more stable (less oscillatory) responses. Response times around 5-7 seconds resulted in the least stable (most oscillatory) responses, likely due to excitation of a natural mode of the Oahu power system related to synchronous generator governor-turbine dynamics. The conclusion that faster responses are more stable runs counter to the expectations of some power systems engineers, and hence was an important finding. However, note that the PSS/E model would not capture certain types of interactions, notably subsynchronous torsional interactions. Regardless, due to the high ROCOF of frequency events in low-inertia systems such as Hawaii a relatively fast response (e.g. sub-second) is needed to arrest frequency events.

- Steeper frequency-watt droop slopes provide a stronger response but also tend to be more oscillatory. Droop slopes in the 4-5\% range represent a reasonable compromise between strength and stability.

- A substantial aggregate capacity of frequency-responsive DERs (at least several tens of $\mathrm{MW}$ ) is needed to have a noticeable stabilizing effect on frequency. 
Table 5: Oahu 2019 Light Spring Case Generation Mix

\begin{tabular}{|l|c|c|}
\hline & Instantaneous Power (MW) & $\begin{array}{c}\text { Instantaneous power } \\
\text { proportion }\end{array}$ \\
\hline Total Generation & 920 & \\
\hline Synchronous Generation** & 262 & $28.5 \%$ \\
\hline Wind & 64 & $7.0 \%$ \\
\hline Station PV & 128 & $13.9 \%$ \\
\hline \begin{tabular}{l} 
Distributed PV* \\
\hline Energy Storage
\end{tabular} & 467 & $50.8 \%$ \\
\hline $\begin{array}{l}\text { Renewables (Total) } \\
\text { *322 MW of distributed PV was equipped with frequency-watt control. }\end{array}$ \\
$\begin{array}{l}\text { **Total system inertia constant is 3.41 MW.s/MVA, and total inertial kinetic energy } \\
\text { of generation is 3.1 GW.s }\end{array}$ \\
\hline
\end{tabular}

\subsection{Simulation of Underfrequency Events}

Many PV systems being installed in Hawaii now include battery energy storage. The batteries are generally being used to absorb PV generation during the daytime and the use the energy to power loads in the evening and at night. This pattern of operation makes economic sense under the currently-available DER tariffs, which either require or incentivize customer PV generation to be used to supply the customer's local loads. While the primary purpose of this behind-themeter energy storage is load shifting (in addition to providing backup power during outages), it could also enable underfrequency response. Indeed, once IEEE 1547-2018 is implemented it will require available DER energy to be deployed for frequency-watt response during underfrequency events.

In anticipation of the growing quantity of distributed energy storage, various simulations of DER underfrequency response were run to examine its effect on bulk system frequency dynamics. Hawaiian Electric's PSS/E model of Oahu for the 2019 heavily-loaded summer-season planning case was used for these simulations. This dispatch scenario represents the worst-case underfrequency event because the largest generator, the AES coal plant, is dispatched to its full $210 \mathrm{MW}$ capacity, resulting in a large contingency relative to the total load of $1095 \mathrm{MW}$. In this model the aggregate distributed PV power was $458 \mathrm{MW}$, which represents about $42 \%$ of the load. DERs were aggregated at their respective transmission buses and the PSS/E user-defined model was used to model the aggregated DERs based on the block diagram shown in Figure 24. 
The heavy summer case has nine active synchronous generators with a total output of $469 \mathrm{MW}$, two active synchronous condensers, and transmission-connected renewable generators with a total output of $168 \mathrm{MW}$ (15\% of load). $316 \mathrm{MW}$ of the distributed PV inverters were equipped with the developed UDMs and thus enabled to participate in frequency response. The rest of the distributed PV inverters represent legacy PV and were assumed to inject constant power regardless of the frequency on the short time scales simulated here. For this simulation study, the initiating contingency is the loss of the $210 \mathrm{MW}$ AES plant, which causes a loss of $25 \mathrm{MW}$ of local load connected at the same bus. This contingency was identified by Hawaiian Electric as a worst-case loss of generation scenario.

Table 6: Oahu 2019 Heavy Summer Case Generation Mix

\begin{tabular}{|l|c|c|}
\hline & Instantaneous Power (MW) & $\begin{array}{c}\text { Instantaneous power } \\
\text { proportion }\end{array}$ \\
\hline Total Generation & 1095 & $100 \%$ \\
\hline Synchronous Generation** & 469 & $43 \%$ \\
\hline Wind & 35 & $3 \%$ \\
\hline Station PV & 133 & $12 \%$ \\
\hline Distributed PV* & 458 & $42 \%$ \\
\hline Energy Storage & 0 & $0 \%$ \\
\hline $\begin{array}{l}\text { Renewables (Total) } \\
\text { *316 MW of distributed PV was equipped with frequency-watt control. }\end{array}$ \\
$\begin{array}{l}\text { ** Total system inertia constant is 3.58 MW·s/MVA, and total inertial kinetic energy } \\
\text { of generation is 4.1 GW·s }\end{array}$
\end{tabular}

The base frequency-watt parameters used in underfrequency contingency simulations are shown in Table 7 . The value of the frequency measurement time constant, $T_{1}$, was chosen conservatively; it is expected that modern frequency measurement methods can act more quickly. Frequency measurements methods vary between inverter models and are a crucial component of effective frequency response. In the sensitivity analyses described below, the appropriate parameters from Table 7 were varied to investigate their effects on the Oahu frequency response. 
Table 7: Frequency-watt control base model parameters for PSS/E simulations

\begin{tabular}{|l|l|l|}
\hline Frequency measurement time constant & $T_{1}$ & $0.5 \mathrm{sec}$ \\
\hline Power response time constant & $T_{2}$ & $0.3 \mathrm{sec}$ \\
\hline Underfrequency deadband & $d b_{u f}$ & $-36 \mathrm{mHz}$ \\
\hline Overfrequency deadband & $d b_{o f}$ & $+36 \mathrm{mHz}$ \\
\hline Underfrequency droop slope & $K_{u f}$ & $4 \%$ \\
\hline Overfrequency droop slope & $K_{\text {of }}$ & $4 \%$ \\
\hline Nominal frequency & $f_{0}$ & $60 \mathrm{~Hz}$ \\
\hline
\end{tabular}

Recall that the time constant for a first-order transfer function is about one third of the response time, so a power response time constant of 0.3 seconds results in about a one second response time. Most hardware inverters' time responses were found to be faster than the default values shown above, which is preferable for system stability on Hawaii (though one inverter tested had a much slower response time). For example, in most PHIL tests the measurement response time of the modeled inverters was set to $0.15 \mathrm{~s}$ and the power response time was set to $0.125 \mathrm{~s}$, and these values were found to match reasonably well with the behavior of hardware Inverter 1 . Inverter 2 was modeled as having a second-order time response with an open-loop response time on the order of 0.5 seconds. The manufacturer of Inverter 3 states a frequency-watt response time of $0.5 \mathrm{~s}$, which includes both the measurement response time and the power response time. Inverter 3 is certified to UL 1741 SA with this frequency-watt time response. Inverter 4 was found to have a frequency-watt response time of just under 0.2 seconds. The frequency-watt response time of Inverter 5 was much slower at about seven seconds, which may be acceptable in some large interconnections but is not fast enough to mitigate Hawaii frequency events and could even result in frequency oscillations if deployed widely in Hawaii. In summary, response times defined by the time constants shown in Table 7 are within most hardware inverters' capabilities, but some inverters need to be updated to enable response times appropriate for Hawaii.

Figure 26 shows the baseline heavy-summer worst-case $\mathrm{N}-1$ contingency event for Oahu. Underfrequency load-shedding (UFLS) points are labeled for reference. In addition, the figure shows the tripping of about $50 \mathrm{MW}$ of legacy PV systems set to disconnect within 0.16 seconds of the moment frequency reaches $59.3 \mathrm{~Hz}$ (the IEEE 1547-2003 setting), which of course exacerbates the underfrequency event significantly. Figure 27 shows the total system load during the same event, including the magnitudes of each UFLS block. Figure 28 shows the total distributed PV power during the event. Note that each UFLS block also sheds at least $10 \mathrm{MW}$ of load (and up to $50 \mathrm{MW}$ ) connected to the same transmission bus, reducing the effectiveness of the UFLS scheme. The amount of PV shed due to UFLS is in the range of one quarter to one half of the load for each block. 


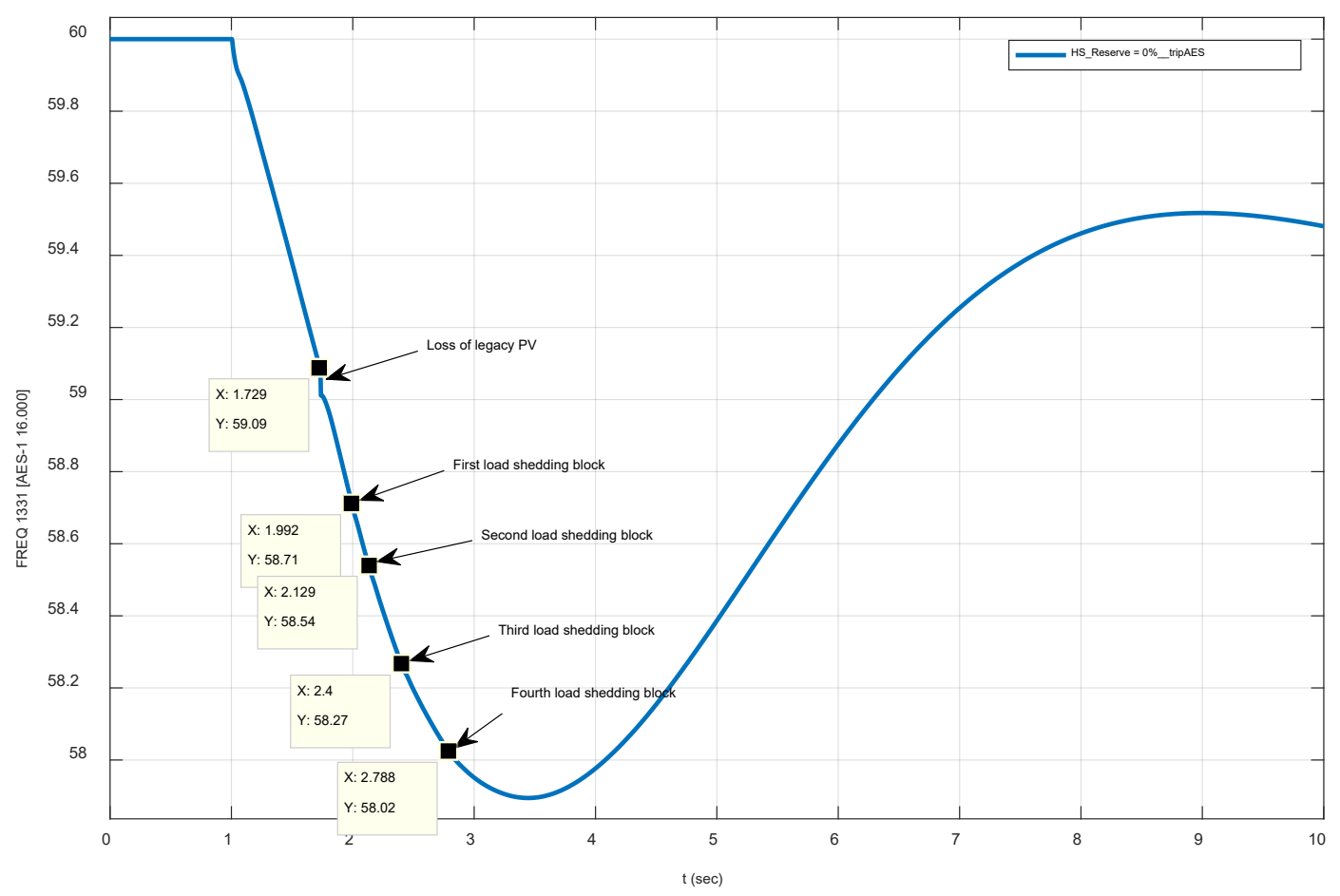

Figure 26: Baseline underfrequency event with no frequency-responsive DERs

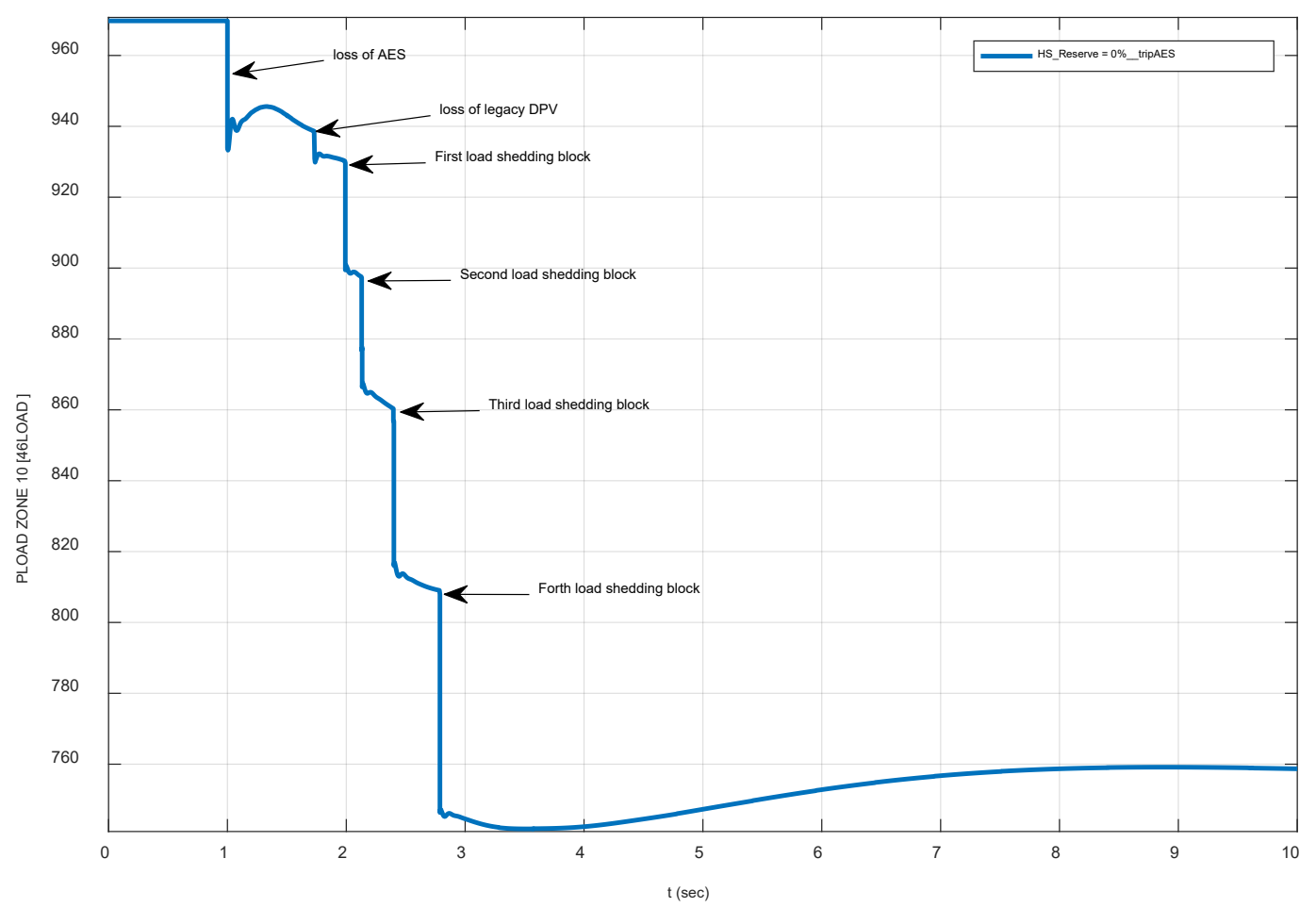

Figure 27: Total load during baseline underfrequency event 


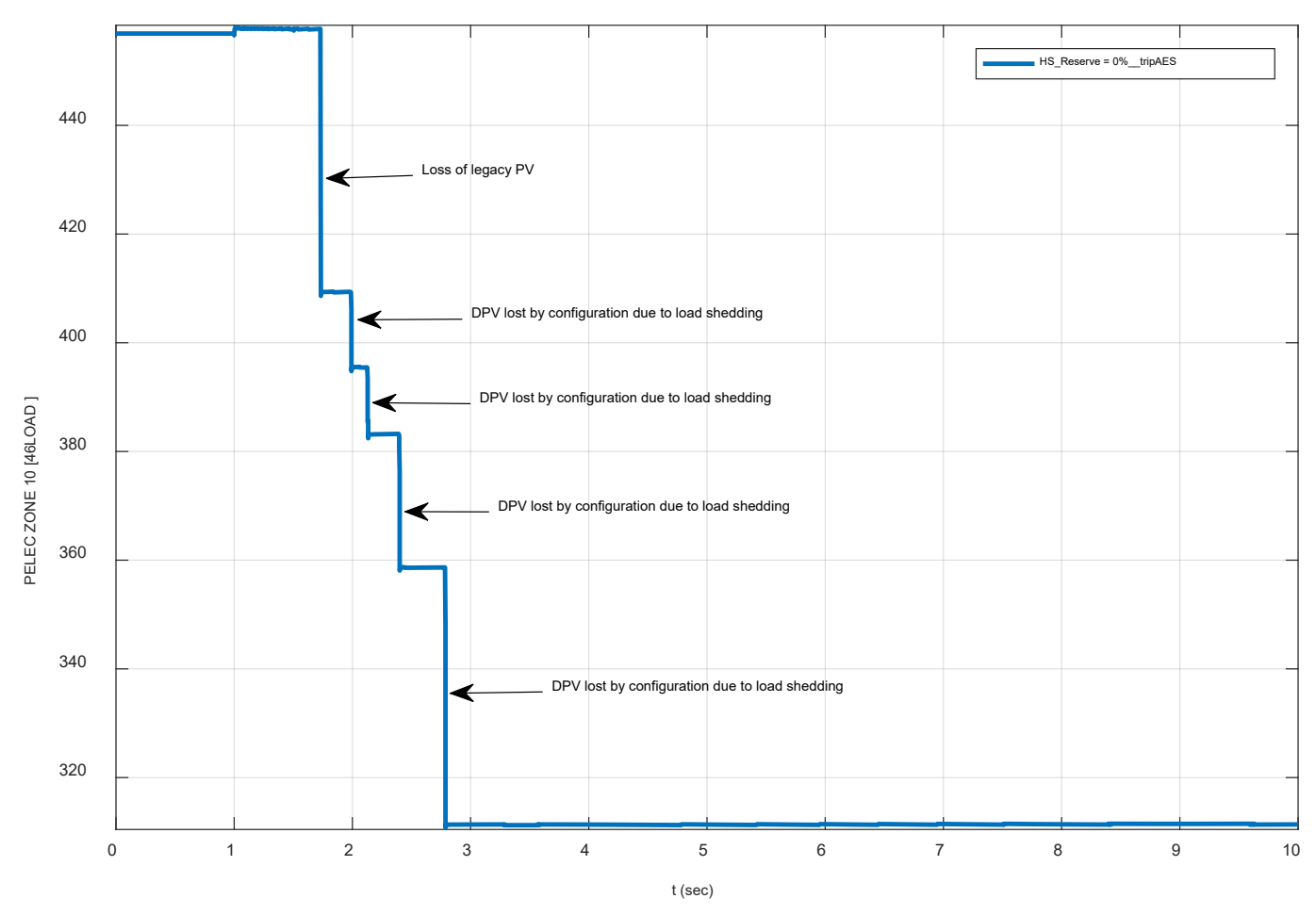

Figure 28: Total distributed PV power during baseline underfrequency event

Figure 29 shows a series of simulations that are the same as the baseline frequency event except that varying levels of DER power are made available to enable upward response. For the purposes of this simulation, the DER reserve power could come from energy storage systems or from PV operating below its present maximum available power level; both types of resources appear the same from a bulk power system perspective. As the DER power reserve increases from zero to $30 \%$ of the DER rated power, the frequency nadir increases (meaning that the severity of the frequency event is mitigated, as desired). Figure 30 shows the DER power at one specific bus that has only advanced inverters connected (no legacy inverters). This allows a comparison of the advanced DER power response under varying reserve levels. While Figure 30 shows the DER power at only one bus, the power response is representative of the response of all frequency-responsive DER in the system because the frequency is nearly uniform across the system. As expected, the advanced DERs increase their power output in response to the frequency event, though they are constrained by their maximum available power level. Figure 31 shows the total DER power during the same series of frequency events. Interpreting the total DER power is complicated because it is influenced by the legacy PV trip, the load shedding events, and the frequency-watt droop response. For lower levels of reserve power, the load shedding tends to dominate, so the net change in DER power is a drop of about $100 \mathrm{MW}$, but for higher levels of reserve power the DER response is enough to avoid triggering one or more UFLS blocks, so more PV remains online. The effect of DER frequency-watt response in reducing load shedding can be seen directly in Figure 32: in the case with $30 \%$ reserve, two UFLS blocks are avoided, and in the cases with $10 \%$ to $20 \%$ reserve, one UFLS block is avoided. The differences in UFLS result in differences in the final frequency value reached at the end of the simulation, with the $30 \%$ reserve case resulting (perhaps unexpectedly on initial examination) in a slightly lower final frequency. Despite this, the $30 \%$ reserve case still results in the most desirable outcome overall because it results in the least 
load shed (i.e. the greatest number of customers remaining with power). In general, the DER frequency response tends to improve the system-wide outcome.

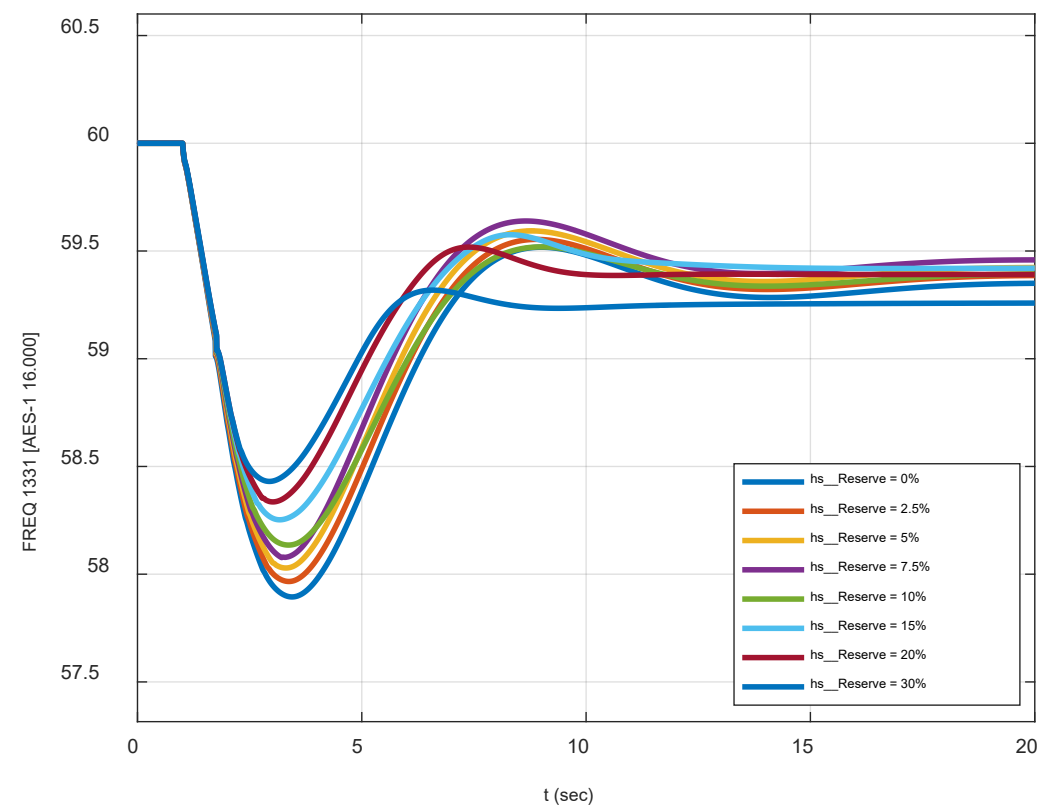

Figure 29: Frequency response with 4\% DER droop slope and varying levels of DER reserve power

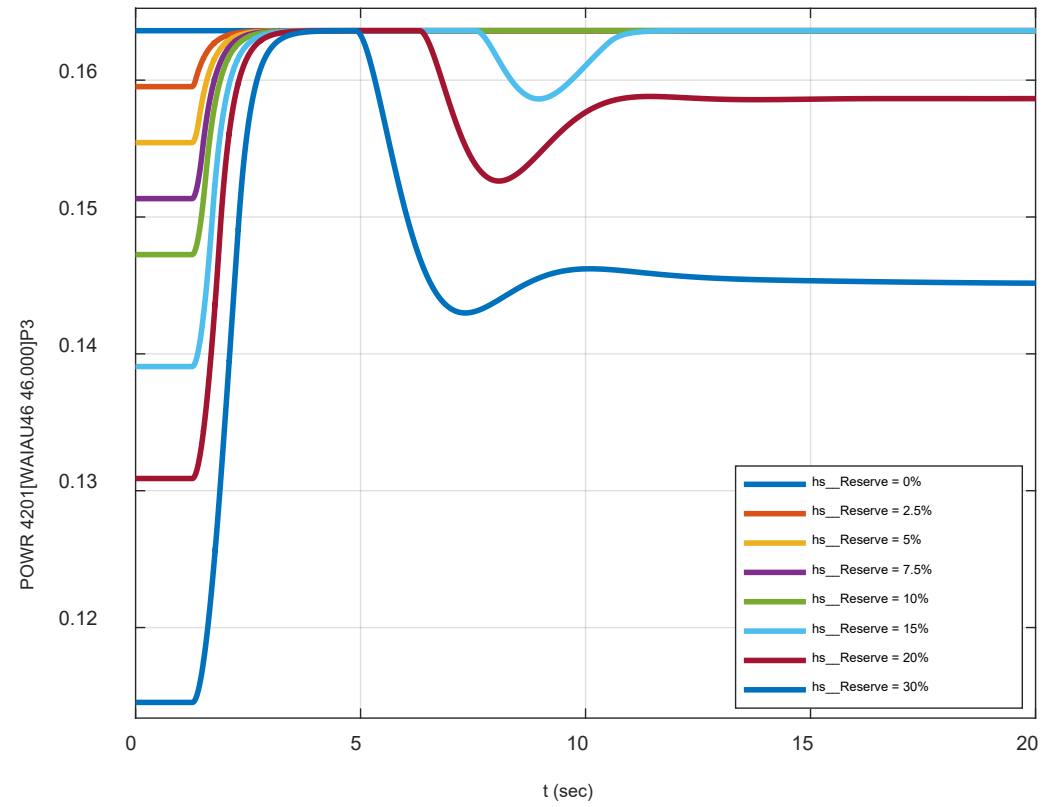

Figure 30: Comparison of DER power levels for frequency events with 4\% DER droop slope and varying levels of DER reserve power 


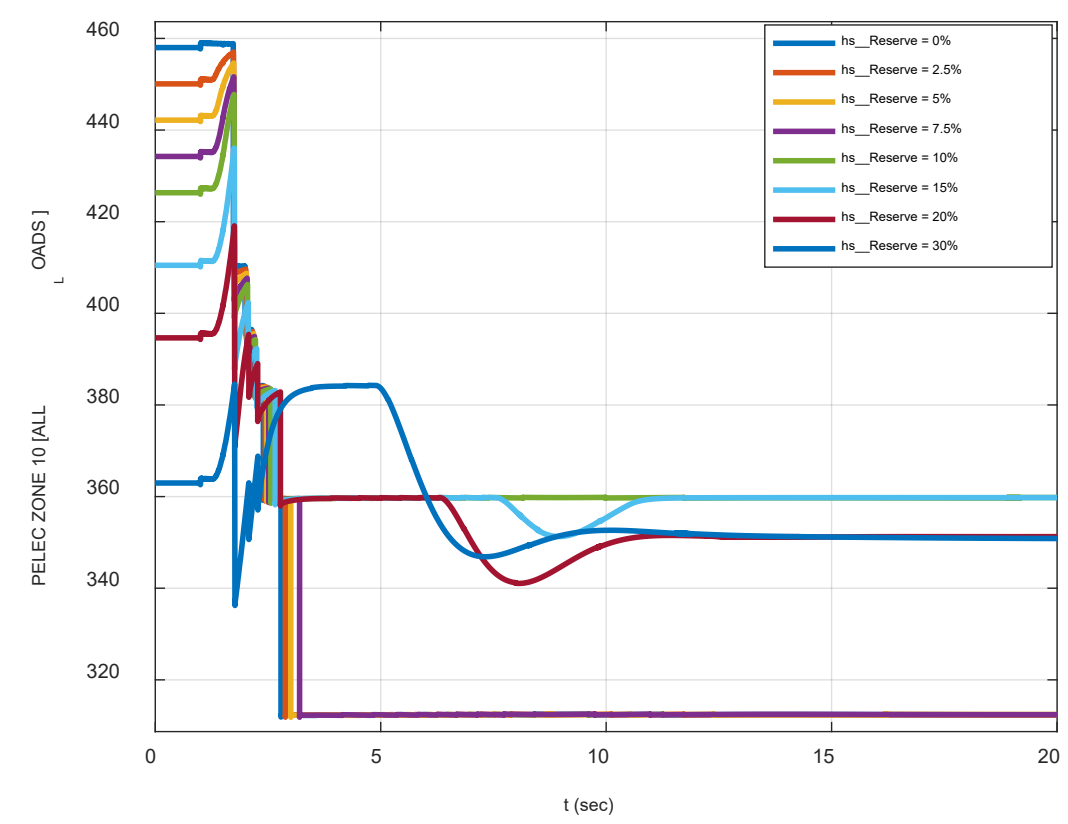

Figure 31: Total distributed PV power for frequency events with 4\% DER droop slope and varying levels of DER reserve power

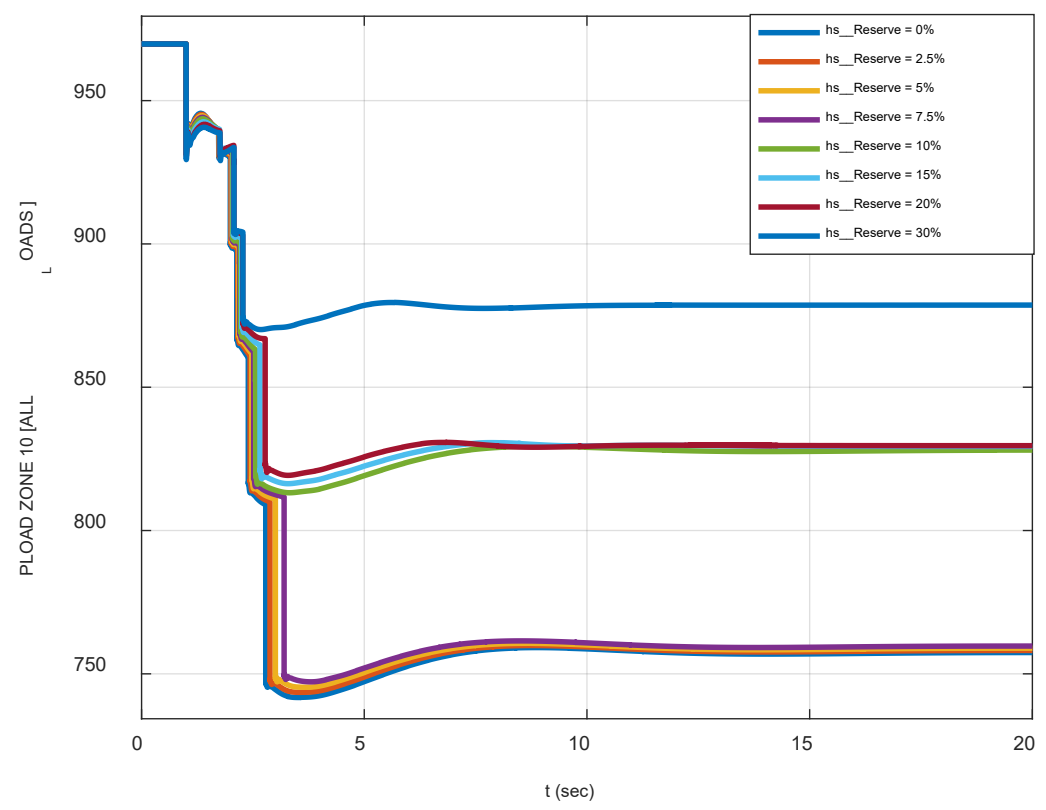

Figure 32: Total load for frequency events with $4 \%$ DER droop slope and varying levels of DER reserve power

As expected, the simulations of underfrequency events show that frequency responsive DERs tend to improve the system response to contingency events. It would take some time to build up the levels of frequency responsive DERs simulated here and build operational confidence 
that the DERs will respond as desired, especially in the case of DERs that can respond to underfrequency.

\subsection{Power Hardware-in-the-Loop Testing}

\subsection{PHIL model overview}

To evaluate the hardware inverters in an environment that emulates the dynamics that would be seen on a real Oahu distribution feeder during a frequency event, a real-time model of Oahu's bulk power system and a selected distribution feeder was developed, as seen in Figure 33. The PHIL test setup was very similar to that used in the interim project report [4], with modifications to allow for testing of storage inverters and of a PV inverter capable of responding to underfrequency events (Inverter 3). The real-time model was used to run PHIL tests in which two residential-scale inverters were connected to two nearby distribution secondary locations. In some tests, both hardware inverters were PV inverters, and in other tests, one was a PV inverter and the other was a storage inverter. For cases involving two hardware PV inverters, both the distribution model and the bulk system model were populated with modeled inverters representing a projection of the distributed PV expected to be present in 2019 . For cases involving and a hardware PV inverter and a hardware storage inverter, some of the modeled inverters were replaced with modeled storage inverters. The modeled inverters' frequency-watt response was simulated using a model corresponding to the PSS/E user-defined model (Figure 24). Frequency events similar to those simulated in PSS/E were run using the PHIL setup, and the responses of the hardware inverters, the modeled inverters, the bulk system, and the distribution system were recorded. The operation of the real-time model is summarized here and will be described in detail in a forthcoming publication. 


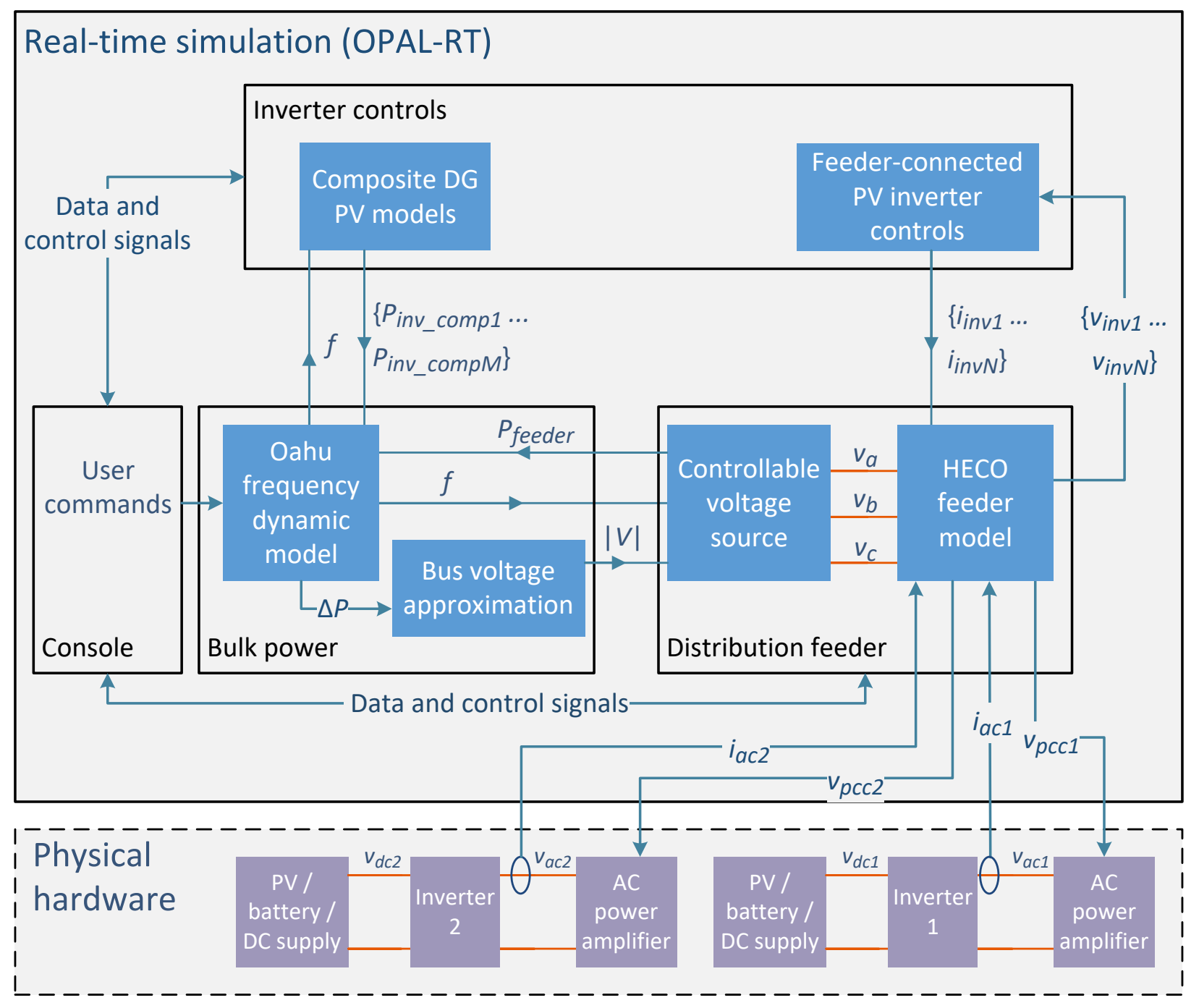

Figure 33: Real-time model of Oahu bulk system and distribution system for PHIL 
The real-time bulk power system model was based on the one described in [28]. The model was modified to better capture the frequency dynamics of the Oahu power system. A schematic of the model is shown in Figure 34. The model solves for frequency directly without solving for electrical power flow. Synchronous generator governor and turbine dynamics are captured, as are the power-frequency dynamics of DER inverters, wind generation, transmission-connected $\mathrm{PV}$, and load shedding. The inertia of the various generators is represented in aggregate, so the individual dynamics of the rotating generators are aggregated together, as in [29]. The aggregate bulk power system model was tuned to match the dynamics of Hawaiian Electric's PSS/E model with good but not perfect accuracy by tuning the transfer functions of the governors and turbines.

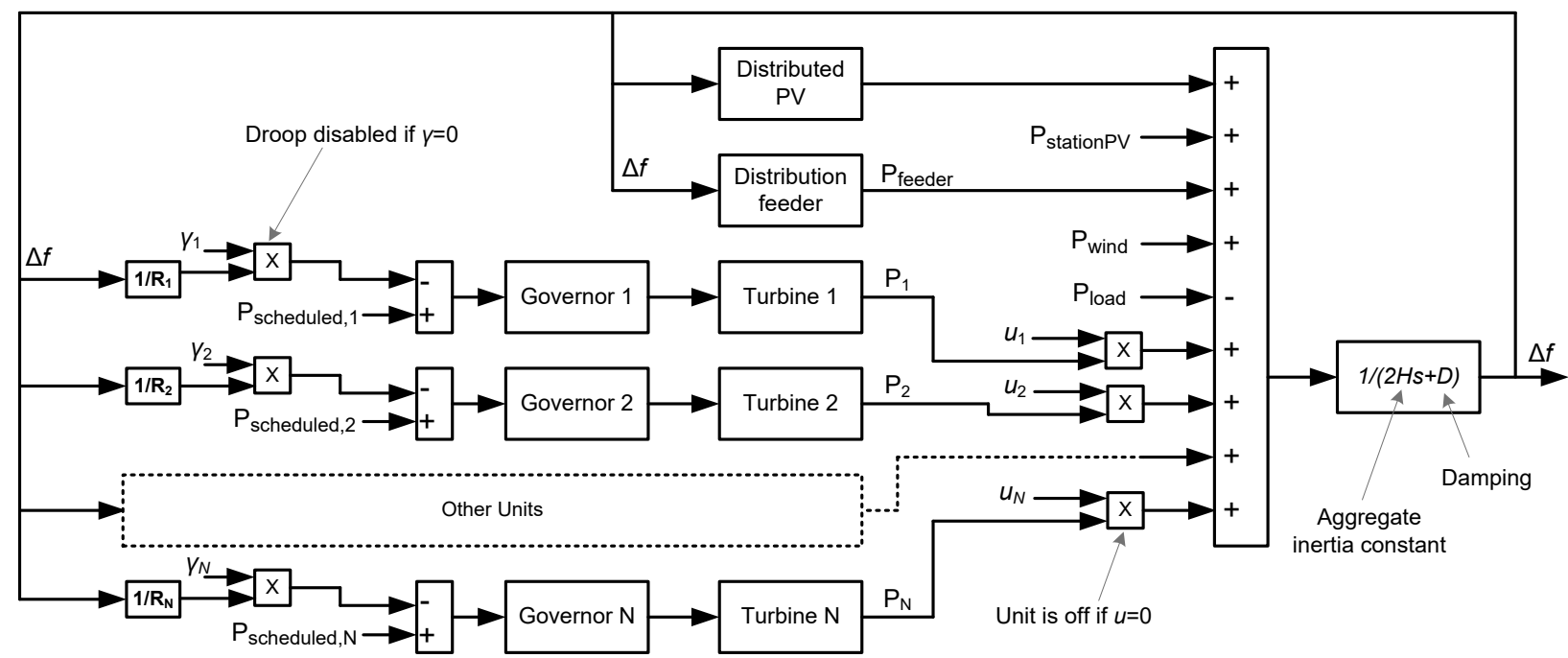

Figure 34: Oahu bulk power system frequency dynamic model

The frequency calculated in the bulk system model was used to drive the voltage at the head of a reduced order distribution model, as originally proposed in [30]. The reduced order distribution model was developed using the technique described in [31] from a Hawaiian Electric Synergi model of an Oahu distribution feeder with a high level of distributed PV. The overall approach is similar to that used in [28], but the following additional elements were added to better capture the realistic dynamics of a frequency event:

- Underfrequency load shedding dynamics were added to the bulk system model, including the effects of load shedding on DER power production. When load is shed during daytime, a significant amount of PV generation is often also shed, as observed in Section 4.4.

- Aggregate models of the distributed PV and storage inverters connected to the rest of the system were added to the model. Four types of distributed PV models were added:

A. Legacy PV with no frequency ride-through and no frequency-watt control

B. PV inverters with underfrequency ride-through but no overfrequency ride-through and no frequency-watt control 
C. Grid supportive PV inverters with full frequency ride-through and frequency-watt control with the power response modeled with a first-order transfer function

D. Grid supportive PV inverters with full frequency ride-through and frequency-watt control with the power response modeled with a second-order transfer function

- For cases including distributed storage, the fourth type of aggregate PV inverter model above was replaced with aggregate storage inverter models. The storage inverter models were configurable to reflect various constraints on storage inverter operation. For example, as noted in Section 2.2, at the time of this work the storage inverter used here was only capable of frequency-watt control when in discharging mode and could only reduce power (meaning it could only respond to overfrequency events, and only in discharge mode). However, because IEEE 1547-2018 requires distribution-connected storage inverters to be capable of underfrequency response, some simulation cases were included where storage responds to underfrequency.

- The frequency ride-through capabilities of the modeled inverters represent the present and expected future DERs on the Oahu power system, and the aggregate ratings of each type. The storage inverters were modeled with full ride-through capabilities, reflecting the requirements for all new DERs. PV types 3 and 4 are intended to model the two types of PV inverter frequency-watt response seen in laboratory testing (firstand second-order dynamics). Because most inverters tested showed frequency-watt responses that can be well modeled with first order transfer functions, most frequency responsive PV was modeled using Type 3 . Most tests were conducted assuming that all of the fully ride-through capable PV in 2019 was also capable of frequency-watt control. This assumption overestimates the amount of frequency-watt capable PV present in 2019 , so can be taken to approximately represent future years. In addition, note that none of the distributed PV or storage in Hawaii currently provides underfrequency response, so these scenarios are purely hypothetical.

- The same four types of PV inverter models were also added to each node in the distribution system model with power rating that approximate the expected aggregate power rating of each type of inverter in 2019. For experiments involving the storage inverter, the storage inverter model was substituted for Type 4 PV, as in the bulk system model.

- Power changes in the modeled distribution system are incorporated into the bulk power system model frequency dynamics, as shown near the top of Figure 33.

- A method was developed for approximating the changes in voltage that occur on the selected distribution system due to bulk power system transients during load rejection events and generation loss events. This allows the model to expose the hardware inverters to realistic fast voltage transients that occur during frequency events, which better validates the inverters' ability to remain online and provide frequency support during such transients. The details of the method for approximating voltage transients associated with frequency events will be described in a subsequent publication.

Table 8 summarizes the settings used for each of the modeled inverters used in the real-time simulation. 
Table 8: Modeled inverter settings for PHIL tests

\begin{tabular}{|c|c|c|c|c|c|c|}
\hline Inverter Type & $\begin{array}{l}\text { HECO } \\
\text { PSS/E } \\
\text { model }\end{array}$ & $\begin{array}{c}f-W \\
\text { Capable? }\end{array}$ & $\begin{array}{c}f-W \\
\text { dynamics }\end{array}$ & $\begin{array}{l}\text { Power } \\
\text { Factor }\end{array}$ & $\begin{array}{l}\text { Underfrequency } \\
\text { Trip Setting }\end{array}$ & $\begin{array}{c}\text { Overfrequency } \\
\text { Trip Setting }\end{array}$ \\
\hline $\begin{array}{l}\text { A: PV, no ride- } \\
\text { through }\end{array}$ & DGPV1 & No & NA & 1.00 & $\begin{array}{l}<59.3 \mathrm{~Hz} \text { for } \\
105 \mathrm{~ms}^{*}\end{array}$ & $\begin{array}{l}>60.5 \mathrm{~Hz} \text { for } \\
105 \mathrm{~ms}^{*}\end{array}$ \\
\hline $\begin{array}{l}\text { B: PV, low- } \\
\text { frequency ride- } \\
\text { through only }\end{array}$ & DGPV2 & No & NA & 1.00 & $\begin{array}{l}<57.0 \mathrm{~Hz} \text { for } \\
110 \mathrm{~ms}^{*}\end{array}$ & $\begin{array}{l}>60.5 \mathrm{~Hz} \text { for } \\
110 \mathrm{~ms}^{*}\end{array}$ \\
\hline $\begin{array}{l}\text { C: PV, full ride- } \\
\text { through }\end{array}$ & DGPV3 & Yes & $\begin{array}{l}\text { First- } \\
\text { order }\end{array}$ & -0.95 & $\begin{array}{l}<57.0 \mathrm{~Hz} \text { for } \\
20.5 \mathrm{sec}\end{array}$ & $\begin{array}{l}>63.0 \mathrm{~Hz} \text { for } \\
20.5 \mathrm{sec}\end{array}$ \\
\hline $\begin{array}{l}\text { D: PV, full ride- } \\
\text { through, } 2^{\text {nd }} \text {-order } \mathrm{f}- \\
\text { W response }\end{array}$ & DGPV3 & Yes & $\begin{array}{l}\text { Second- } \\
\text { order }\end{array}$ & -0.95 & $\begin{array}{l}<57.0 \mathrm{~Hz} \text { for } \\
20.5 \mathrm{sec}\end{array}$ & $\begin{array}{l}>63.0 \mathrm{~Hz} \text { for } \\
20.5 \mathrm{sec}\end{array}$ \\
\hline $\begin{array}{l}\text { E: Distributed } \\
\text { storage }\end{array}$ & NA & Yes & $\begin{array}{l}\text { First- } \\
\text { order }\end{array}$ & -0.95 & $\begin{array}{l}<57.0 \mathrm{~Hz} \text { for } \\
20.5 \mathrm{sec}\end{array}$ & $\begin{array}{l}>63.0 \mathrm{~Hz} \text { for } \\
20.5 \mathrm{sec}\end{array}$ \\
\hline
\end{tabular}

Inverters classified as "DGPV3" (sometimes written as "PV3") in the PSS/E models were split into two inverter types in the real-time models, referred to as $C$ and $D$. The distinction is that type $C$ inverters have a first-order frequency-watt response and type $D$ inverters have a secondorder response. For the tests involving distributed battery systems, PV4 was replaced by a storage inverter model. Most tests were run with $97.6 \%$ percent of DGPV3 inverters as type C (inverters having first-order time response) response and $2.4 \%$ of DGPV3 inverters given type D ( $P V$ inverters having second-order time response or storage inverters having $1^{\text {st }}$ order time response).

\subsection{Selected PHIL test results}

In the previous report from this project, several PHIL test results of PV inverter responses to overfrequency events were presented [4]. These tests demonstrated that the inverters performed frequency-watt control as expected, helping to mitigate the overfrequency events by quickly reducing output power. The inverters did not show any adverse interactions with each other or with the power system. In addition, the inverters remained online throughout the severe frequency events and the associated fast voltage transients. The responses of the hardware inverters matched well with the responses of the modeled inverters, confirming the validity of the relatively simple inverter models used here and in the PSS/E simulations. In addition, the response of the PHIL real-time grid model matched well with the PSS/E results.

One update to the above information is that, as noted in Section 2.1, one of the additional PV inverters tested has a frequency response time that is too slow to be effective, so it should not be assumed that all PV inverters will have adequate response times.

Several new PHIL results are presented in this report. New types of experiments include:

- $\quad$ Storage inverter response to frequency events 
- PV inverter response to underfrequency events.

\subsubsection{Battery inverter}

A residential-scale battery inverter (Inverter 6) was used to conduct several tests of the ability of distribution-scale storage inverters to provide grid support during frequency testing. Unfortunately, at the time of testing (late 2017), most commercially available residential scale battery inverters had limited ability to autonomously respond to frequency events, at least using production firmware. As noted in Section 2.2, Inverter 6 could only respond to frequency events when discharging, and it could only decrease power, making its frequency responsive capability very similar to that of most PV inverters.

Because of these limitations in hardware capability, a relatively small number of PHIL tests of storage inverter frequency response were conducted. These tests use the PHIL setup described in Section 5.1, with the light spring generation mix and the M34 distribution feeder model. The tests are initiated by the largest load trip contingency as identified by Hawaiian Electric, which results in an overfrequency event.

Figure 35 shows an example of an overfrequency event tested in PHIL with Inverter 6 providing frequency-watt control. Type D PV inverter models in the real-time simulations were replaced with Type $\mathrm{E}$ (storage inverters). The storage inverter models used a first order time response with a fixed delay of 1 second, reflecting the observed hardware response of Inverter 6 . The stepwise response characteristic was not modeled directly in the real-time model. Instead, it was assumed that different inverters would update their power levels at different times such that the aggregate response of many inverters would follow a smoother trajectory. This assumption that diversity in inverter step response would lead to a smooth (non-stepwise) aggregate response should be validated through field data or other means. The hardware inverter and the modeled inverters of Type $C$ and Type E were set with a 3\% droop slope. The load contingency occurs at time 2 seconds. The grid frequency oscillates somewhat due to the relatively steep droop slope of the modeled inverters. The modeled Type $E$ inverters and the hardware inverter have a delay in their response. It can be seen that the stepwise power response of the hardware inverter roughly follows the smoother response of the modeled inverters, indicating that the model is an acceptable representation of reality (subject to the assumption that diversity of individual inverter responses would smooth the aggregate response). The relatively slow response time of Inverter 6 may lead to reduced effectiveness of frequency-watt control. In this simulation, the frequency-watt response is dominated by the Type $C$ inverters (PV) in the model, which have a much higher aggregate power than do the Type $E$ inverters (storage). 

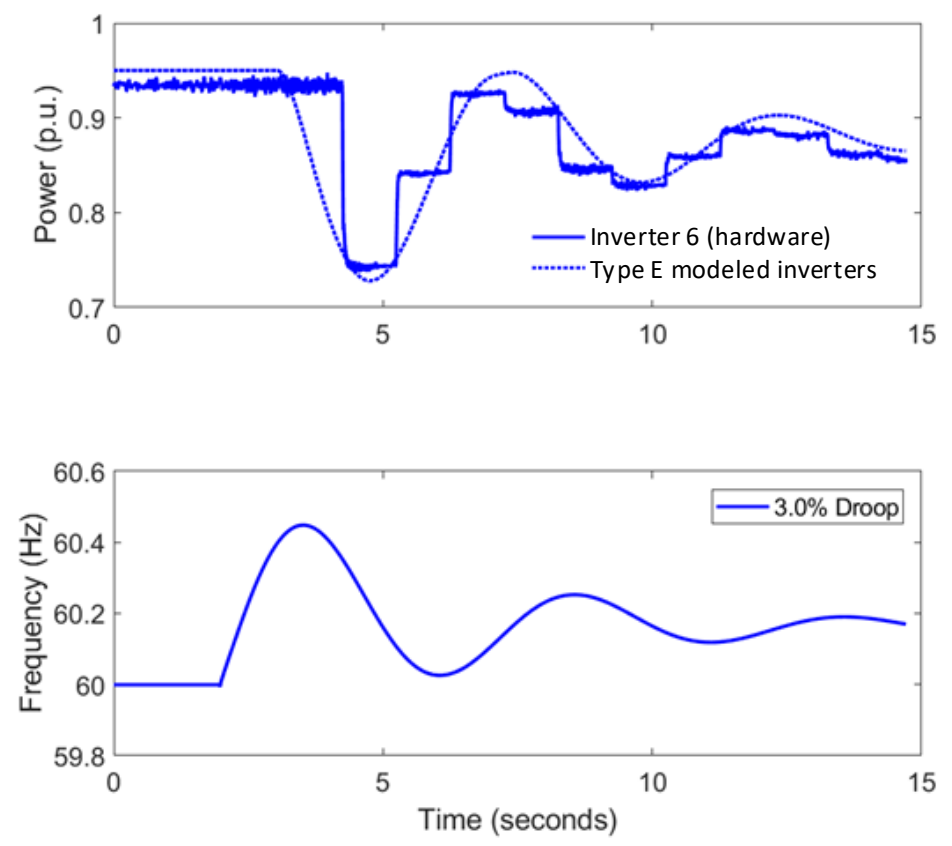

Figure 35: PHIL test of storage inverter response to overfrequency

As storage inverters are updated to comply with IEEE 1547-2018, it would be useful to verify that their responses indeed provide frequency-watt control both during charging and during discharging, and that they have been programmed to respond to both overfrequency and underfrequency events. This is especially important because a high proportion of new PV installations on Hawaii now include battery storage, so the impact of these systems on bulk system stability could become very significant.

\subsubsection{PV inverter underfrequency response}

Inverter $3^{12}$ was used to investigate the PV inverter underfrequency response in PHIL tests. The underfrequency events were initiated in the real time model by tripping the AES coal generator, Oahu's single largest contingency.

Figure 36 shows a series of four underfrequency events with the hardware inverter and the Type C modeled inverters maintaining $30 \%$ of rated power in reserve and configured to provide frequency-watt control, with the droop slope varied between the four simulations. Available PV power was set to $30 \%$ of rated power. Each color in Figure 36 represents a separate test with a different droop setting. The solid lines in all PHIL tests represent measurements of hardware inverter responses, and the dotted lines represent modeled inverter responses. Several conclusions and observations can be made:

- With frequency-watt droop disabled (red traces), the frequency nadir reaches below 57.9 $\mathrm{Hz}$, causing Type A legacy PV to trip and four blocks of load shedding to occur.

\footnotetext{
${ }^{12}$ Inverter 3 in this report was referred to as Inverter 1 in the interim report (Hoke et al, 2017).
} 
- In the three cases with frequency-watt enabled (black, green and blue traces), the frequency reaches $58.4 \mathrm{~Hz}$. Legacy Type A PV trips, and three blocks of load shedding occur. As expected, PV power increases as frequency decreases, and the increase is greatest for steeper droop slopes (lower droop percentages).

- For the cases with frequency-watt enabled, the hardware inverter responses match reasonably well with the corresponding modeled inverter responses. Most of the mismatch is due to the fact that the dotted lines represent the sum of all modeled PV power, so power is reduced when load shedding events trip distributed PV. The downward steps in the dotted traces between 3 and 4 seconds are due to the activation of load shedding blocks, which trip the distributed PV systems in those regions, causing reductions the aggregate distributed PV power output. Hence the dotted lines of each color tend to track below the solid lines by a fixed amount following load shedding. The remaining mismatch between hardware inverter power and modeled inverter power is due to the fact that the inverter models are only an approximation of the hardware inverter dynamics.

- Even with frequency-watt control disabled, the hardware inverter shows some changes in power around its pre-event level. This is a characteristic of this particular inverter: its real and reactive power outputs diverge from their steady-state values during fast frequency transients. Because other inverters tested do not show this behavior, and because this brand of inverter represents a small portion of the distributed PV on Oahu, the changes in reactive power are not modeled.

- The downward spike in hardware inverter power around time 3 seconds is due to a voltage transient on the distribution system caused by the tripping of the legacy PV inverters. This is expected and is similar to what may occur in the field if a large number of DERs trip simultaneously on overfrequency. In practice, the slight diversity in actual trip times of different hardware inverters would likely make this voltage transient less severe. The fact that the hardware inverter rode through this transient means that it would likely also do so in the field, as desired.

- No undesired interactions were observed between the various (hardware and modeled) inverters performing frequency-watt control. In addition, the increase in DER power output in response to the underfrequency event did not cause any distribution voltages to exceed ANSI C84.1 Range A (105\% of nominal voltage). No adverse effects on the distribution system were observed.

- The quasi-steady-state frequency at the end of the test window (around 15 seconds) is generally improved with steeper droop slopes. For the case with droop disabled, more load shedding occurs than in the other cases, so the final frequency is comparable to the case with $5 \%$ droop despite the lack of $P V$ response. 


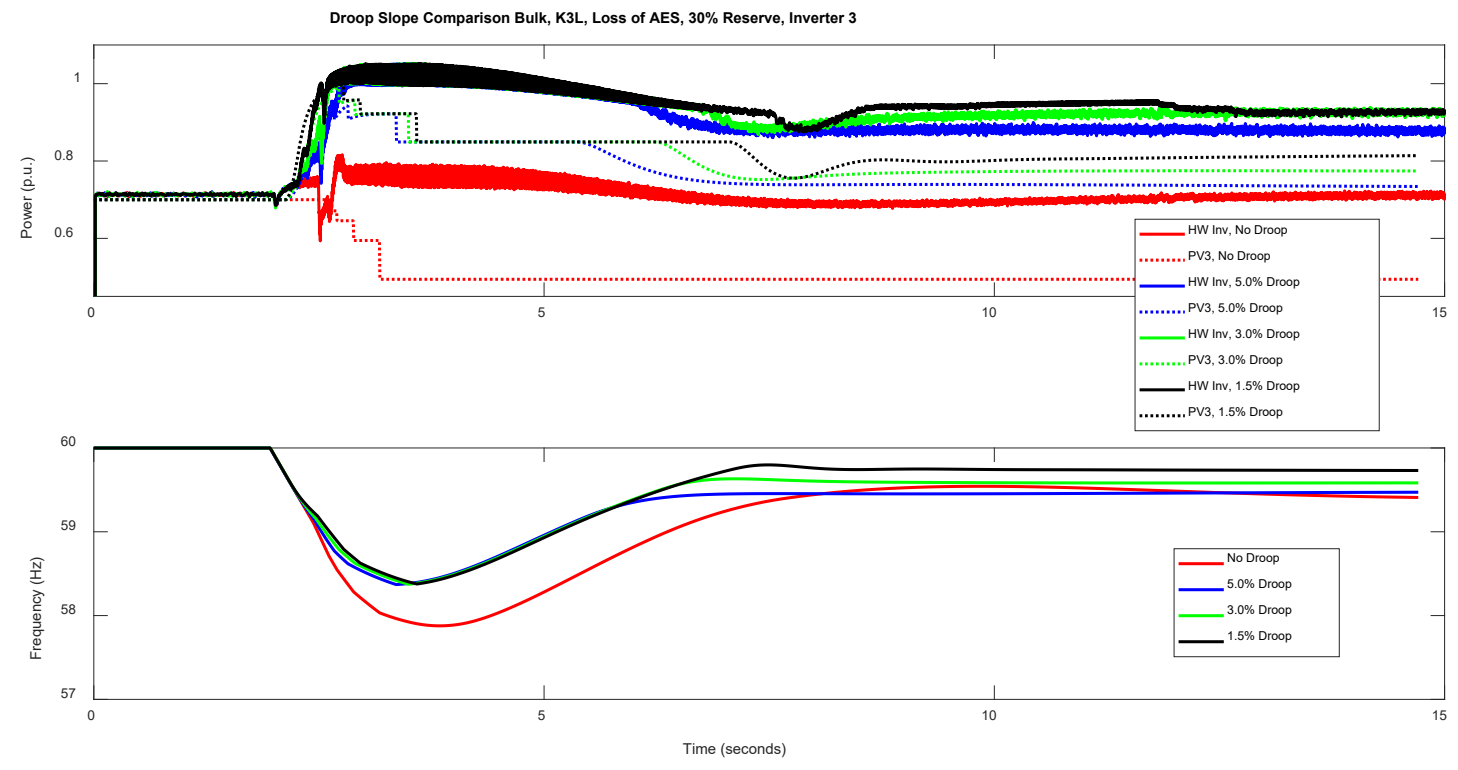

Figure 36: PHIL underfrequency events with varying droop slopes

Figure 37 compares the system response to the AES trip event for varying inverter frequencywatt response times. Type $\mathrm{C}$ modeled inverters were configured to provide frequency-watt control with a droop slope of $5 \%$ and $30 \%$ of rated power in reserve for all tests in this figure. Each color in Figure 36 represents a separate test with a different droop setting. Again, the solid lines represent measurements of hardware inverter responses, and the dotted lines represent modeled inverter responses for Type C distributed PV. Several conclusions and observations can be made:

- The frequency nadir is lower for slower response times, and the system response is more oscillatory. This aligns with the conclusions of PSS/E testing and eigenvalue analysis: faster frequency-watt response times tend to improve system response.

- The simulation does not persist long enough to quantify the settling frequency.

- The simulations with the two fastest inverter response times $(0.38 \mathrm{~s}$ and $1.5 \mathrm{~s})$ result in reduced load shedding relative to the simulations with the longer response times $(5 \mathrm{~s}$ and $10 \mathrm{~s}$ ). The fourth UFLS block is not shed for the faster response times, resulting in about $60 \mathrm{MW}$ of customers remaining online that otherwise would have been blacked out.

- Several conclusions made about Figure 36 appear again Figure 37:

- The tripping of modeled legacy PV inverters on the same distribution circuit as the hardware inverter causes a voltage transient, and the hardware inverter rides through that transient.

- The modeled inverter responses match well with the hardware inverter response when the lost of some modeled inverters due to underfrequency load shedding is accounted for. 


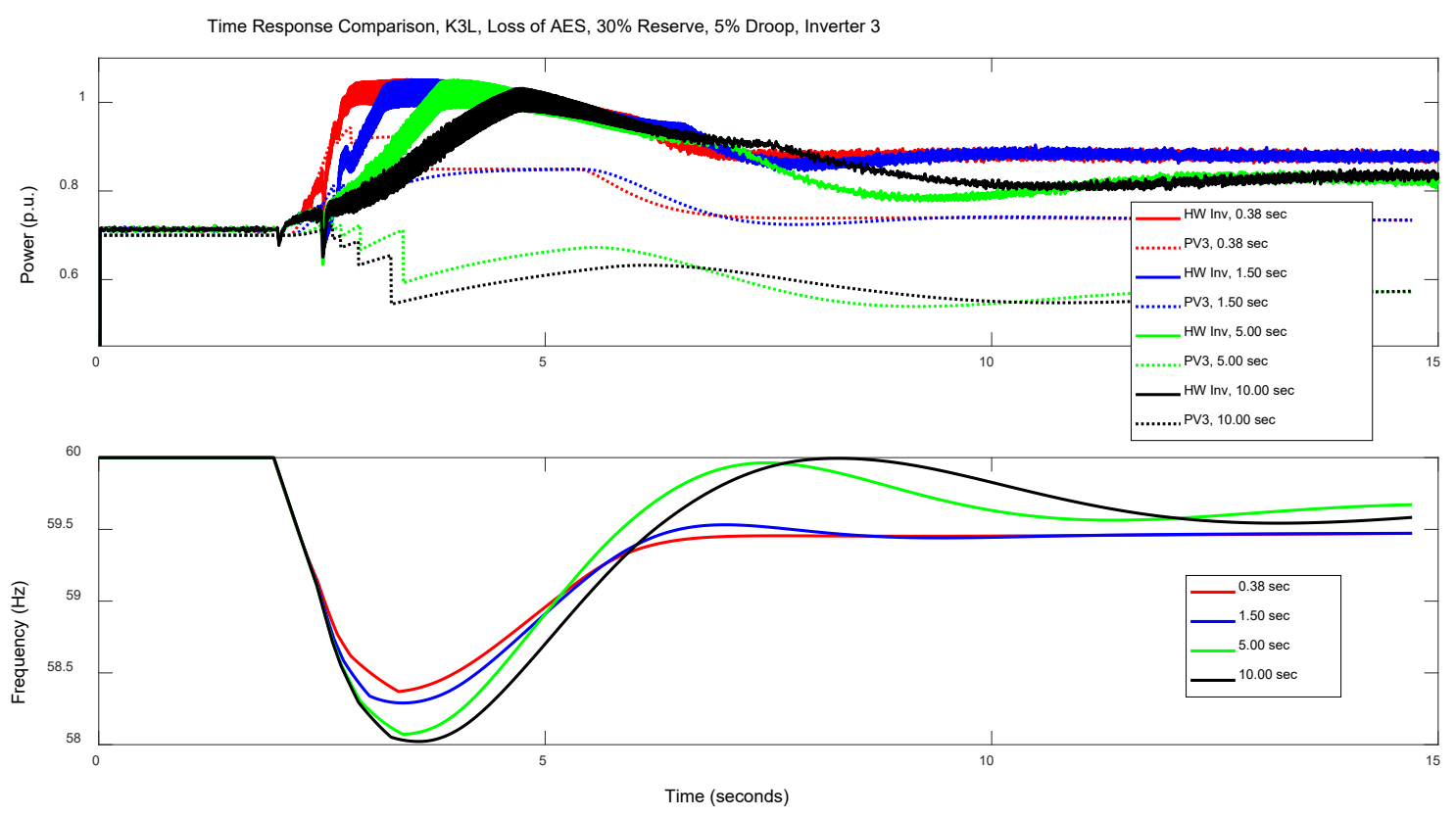

Figure 37: PHIL underfrequency events with varying inverter response times

Figure 38 shows the response of Inverter 3 to the same event with varying frequency-watt deadbands and droop slopes. The three deadbands tested were $0.017 \mathrm{~Hz}$ (red), $0.036 \mathrm{~Hz}$ (blue), and $0.1 \mathrm{~Hz}$ (green). Again, the hardware inverter responses match the modeled inverter responses well but not perfectly. The narrower deadbands slightly improve the nadir and settling frequency.
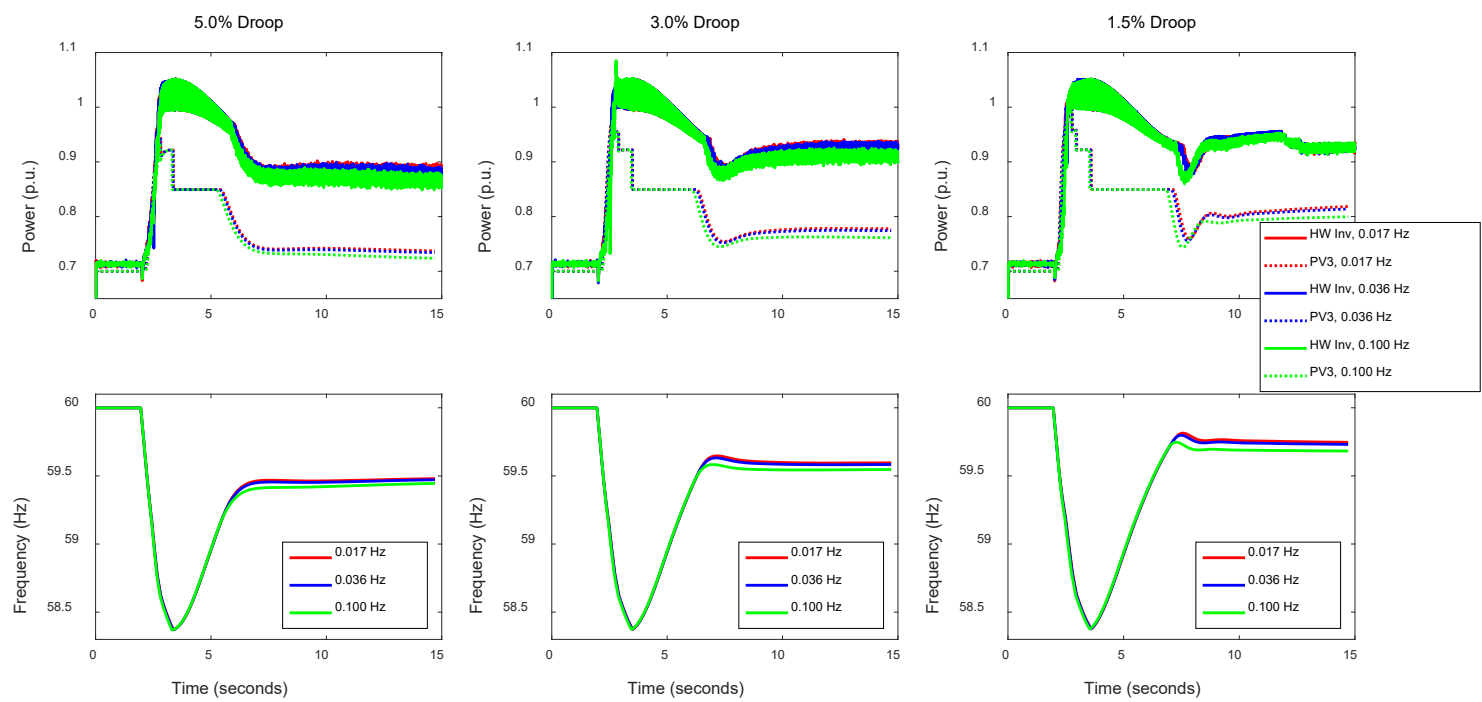

Figure 38: PHIL underfrequency events with varying deadband and droop slope

Figure 39 compares the Oahu frequency response with varying proportions of available PV power held in reserve. The frequency nadir is higher for larger levels of PV held in reserve, and the settling frequency also tends to improve, though the effects of load shedding muddle the picture: When the frequency-watt response succeeds in avoiding load shedding this can result 
in a lower settling frequency (but more customers remain with their lights on, which is a more important metric). Overall, the higher amounts of reserve power result in improved outcomes.
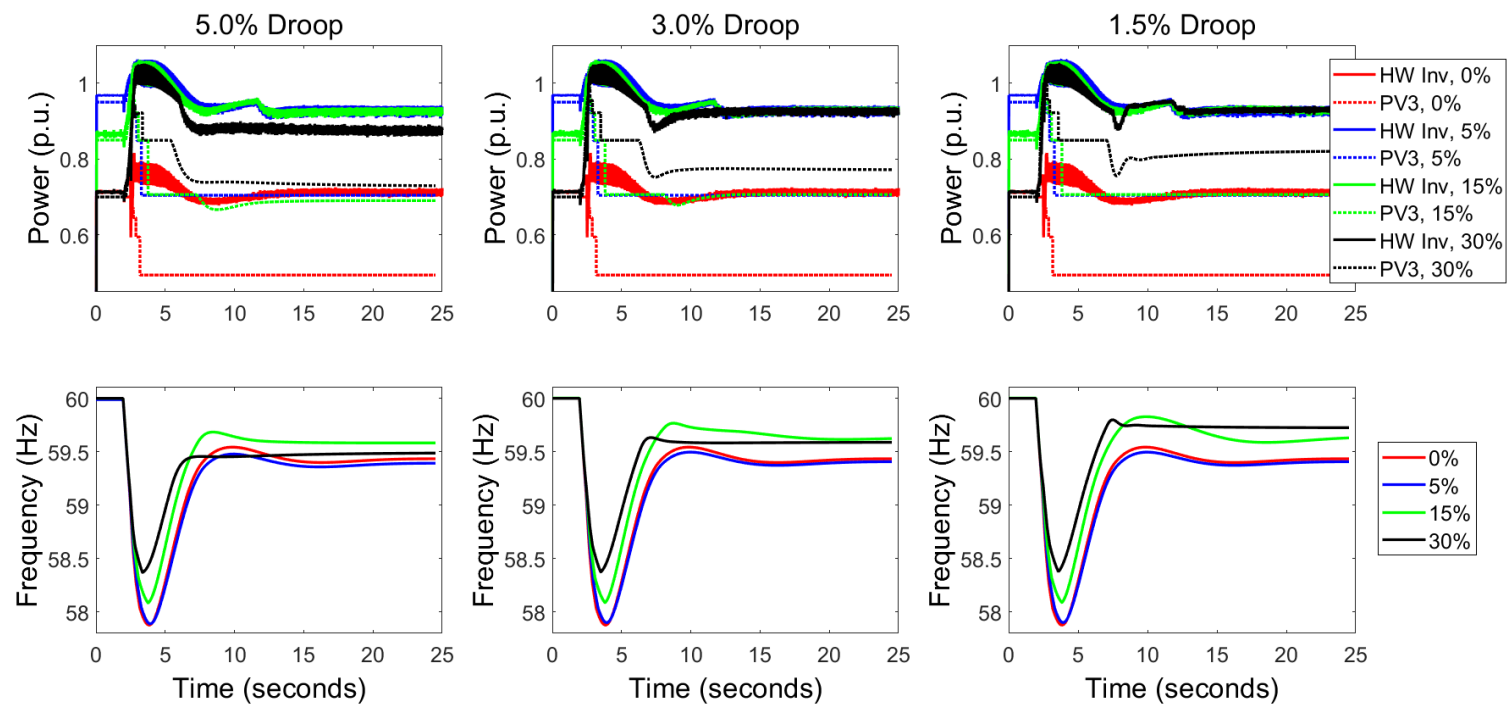

Figure 39: PHIL underfrequency events with varying amounts of available PV power held in reserve and varying droop slopes

As a whole, these PHIL tests demonstrate that if underfrequency response is available from DERs, it can significantly improve frequency response. No distribution system overvoltages or other undesired interactions were observed to result from the DER frequency response. Because inverter dynamics are largely independent of the DC energy source providing the power, this statement is expected to apply to both PV and energy storage.

\subsection{Impact of frequency-watt control on DER energy production}

For DERs that respond to both overfrequency and underfrequency events equally, as would be the case for DERs that include energy storage,,$^{13}$ the net effect on energy production over time is expected to be neutral. This is because the proposed frequency-watt curve is symmetrical around nominal frequency, and secondary frequency regulation maintains steady-state frequency very near $60 \mathrm{~Hz}$ on average. This can be corroborated by analysis of historical frequency data. For example, Figure 40 shows a histogram of the Oahu frequency in May, with the vertical scale displaying the number of seconds frequency was within each histogram bin. The histogram is centered around $60 \mathrm{~Hz}$, as expected.

\footnotetext{
${ }^{13}$ Non-exporting PV systems (e.g. those under the Customer Self-Supply tariff) would also fall info this category, since they can respond to underfrequency events by exporting power.
} 


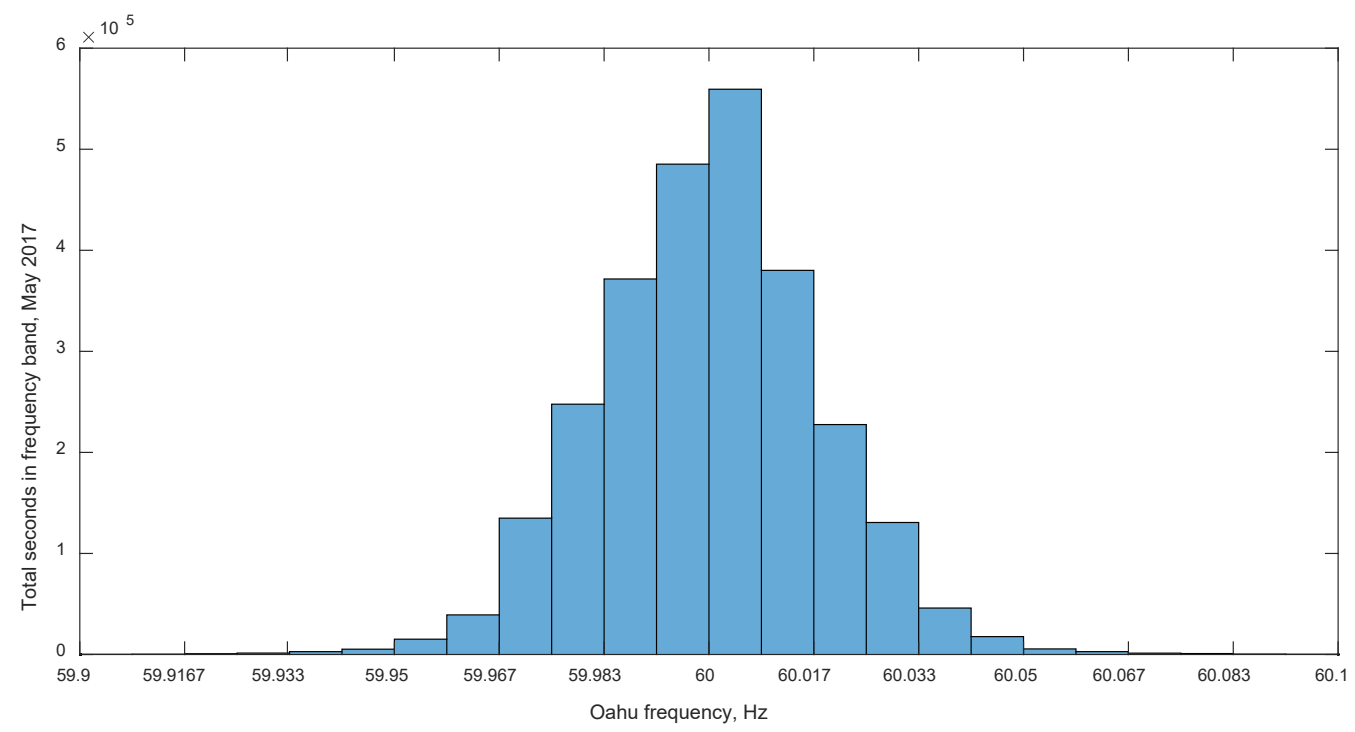

Figure 40: Oahu frequency histogram, May 2017

For DERs that respond to overfrequency events but rarely respond to underfrequency events (such as most exporting PV systems), frequency-watt control will have a small negative impact on energy production. However, that impact will be negligible, as shown in the analysis of historical frequency data presented in [4]. Because frequency is essentially uniform throughout an interconnected power system, this analysis is much simpler than for voltage-related functions. Table 9 shows the estimated PV energy reduction as a percentage of expected monthly energy output for droop slopes of $4 \%$ and $5 \%$ and deadbands between $8.3 \mathrm{mHz}$ and 50 $\mathrm{mHz}$. In all cases the energy reduction is below $0.25 \%$.

Table 9: Estimated impact of frequency-watt control on PV energy production

\begin{tabular}{|c|c|c|}
\hline Deadband $(\mathbf{m H z})$ & Droop (\%) & PV energy reduction (\%) \\
\hline 50 & 4 & $0.006 \%$ \\
\hline 33.3 & 4 & $0.023 \%$ \\
\hline 16.6 & 4 & $0.130 \%$ \\
\hline 8.3 & 4 & $0.290 \%$ \\
\hline 50 & 5 & $0.005 \%$ \\
\hline 33.3 & 5 & $0.019 \%$ \\
\hline 16.6 & 5 & $0.110 \%$ \\
\hline 8.3 & 5 & $0.230 \%$ \\
\hline
\end{tabular}

Based on this analysis, the impact of frequency-watt control on PV energy production is expected to be extremely small for all DERs.

It is possible that as more PV is added to the Oahu power system, overfrequency events will become more common due to lower system inertia, causing the impact of frequency-watt control on PV energy production will increase slightly. However, as shown in this report, when a substantial number of PV systems are performing frequency-watt control, the severity of 
overfrequency events will be reduced. This will tend to mitigate any increasing impact on PV energy production. Overall the impact of frequency-watt control on PV energy production is expected to remain very small.

\subsection{Compatibility of frequency-watt control with fast frequency response (FFR)}

Hawaiian Electric currently offers fast frequency response (FFR) ${ }^{14}$ demand response programs in which DERs or other resources are compensated for providing FFR. FFR is similar to frequency-watt control in that it provides a fast way to help arrest frequency transients by mitigating the generation-load imbalance that caused the event. FFR differs from frequencywatt control in several ways:

- FFR provides a step response in power triggered by a frequency (or ROCOF) threshold, whereas frequency-watt control provides a power response that is proportional to the frequency deviation. (As such, FFR must be used carefully so as not to over-respond and cause an overfrequency event.)

- FFR responds once per frequency event, whereas frequency-watt control continuously adjusts its response moment-to-moment based on changes in grid frequency throughout an event, including responding to cascading events or events with both overfrequency and underfrequency excursions.

- Frequency-watt control (primary frequency response) is typically a requirement of interconnection for all capable generation, whereas FFR is typically an optional service that DER or other resources can elect to provide for compensation.

- Frequency-watt control (primary frequency response) is a basic requirement for momentto-moment balancing of generation and load in all modern interconnected AC power systems. Accordingly, FERC Order 842 [33] and IEEE 1547-2018 [21] require all new generators (both synchronous and non-synchronous, i.e. inverter-based) to provide primary frequency response. In contrast, FFR is an emerging technology employed for specific use cases in only a few locations worldwide, and not typically a requirement of interconnection.

- In IEEE 1547-2018, a DER is required to provide frequency-watt control if power is available, but is not required to maintain upward or downward reserve to enable frequency-watt control. In contrast, a resource providing FFR is required to maintain reserve or it will not get paid for the service.

\footnotetext{
${ }^{14}$ Note the in the Hawaii context and in some other specific locations, FFR is defined as a specific class of grid services characterized by a step-like response in power. In other contexts, the term "fast frequency response" and the acronym "FFR" are defined more broadly to mean any response that rapidly modulates energy injection into and absorption from a power system to help arrest a frequency event, regardless of the specifics of how the amount of energy injected or absorbed is determined. For the purposes of this report, FFR refers to the Hawaii grid services.
} 
A single DER could provide both frequency-watt control and FFR - the two functions are not necessarily mutually exclusive. Specifically, because 1547-2018 does not require DERs to maintain reserve for frequency-watt control, if an FFR response uses up a DER's available reserve the DER is not required to provide frequency-watt control until power becomes available. The trigger points for FFR in Hawaiian Electric's Grid Service Tariff are $59.7 \mathrm{~Hz}$ and $60.1 \mathrm{~Hz}$, both well outside the frequency-watt deadband (currently $60+/-0.036 \mathrm{~Hz}$ ). When frequency is outside the frequency-watt deadband but inside the FFR trigger limits, the DER would provide frequency-watt control. Outside the FFR trigger limits, the DER would provide FFR, and if additional power is available it could also provide frequency-watt control if the frequency-watt curve dictates a larger power than the FFR response does. The frequency-watt and FFR control regions are illustrated in Figure 41 for the case of a DER providing frequencywatt and FFR for both underfrequency and overfrequency events. The underfrequency and overfrequency deadbands for frequency-watt control are denoted $d b_{\mathrm{UF}}$ and $d b_{\mathrm{OF}}$, respectively.

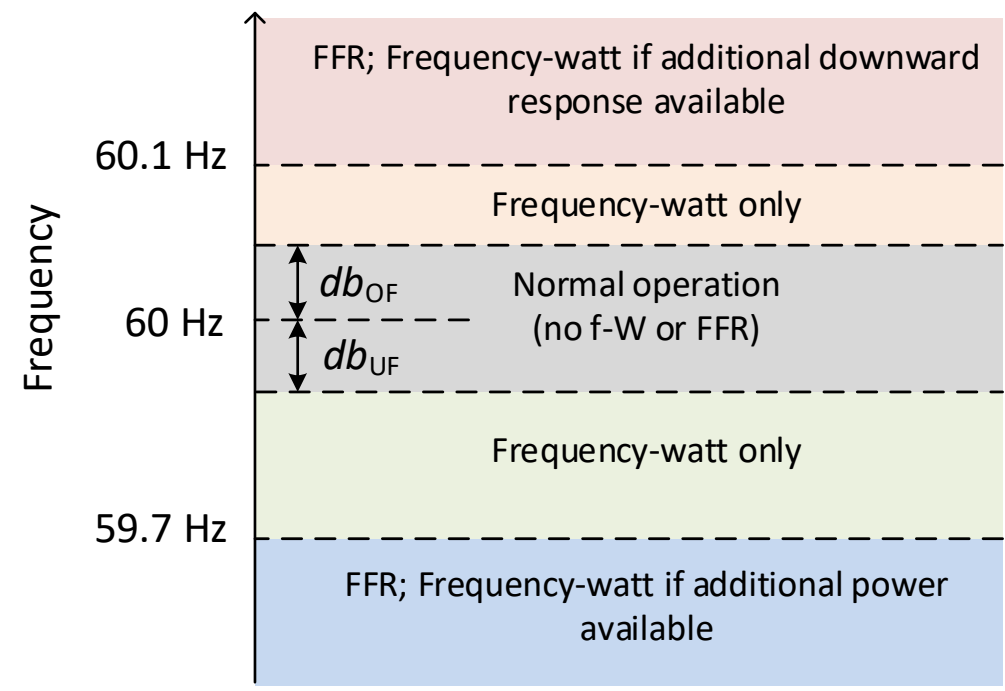

Figure 41: Frequency-watt (f-W) and FFR control regions

Some details would need to be worked out as the FFR tariff was not completely clear on certain points at the time of this writing. For example:

1. When responding to an FFR trigger, should an FFR1 resource start responding within 12 cycles of the trigger, or reach its full designated response within 12 cycles of the trigger? (There is a similar question for FFR2, substituting 30 cycles for 12).

2. When responding to an FFR trigger, the FFR tariff implies that a resource should reach its nominated capability power, but details are unclear. Does the resource need to change its power output to its pre-event power plus its nominated capability? Or does it just need to reach an absolute power level equal to its nominated capability regardless of what power level it was at before the event? If the former, is the pre-event power defined as the power the resource was at when the frequency crossed the trigger?

3. When an FFR response ends, how should the resource transition back to normal operations? Does it ramp back at some controlled rate?

Performing frequency-watt control simultaneously with FFR could also provide a backstop in case an overfrequency event transitions into an underfrequency event (or vice-versa). The FFR 
tariffs requires that the FFR response last 30 minutes, making no provision for backing off the response if needed. Frequency-watt control provides an automated mechanism to back off the response if frequency swings to the other side of nominal during a complex event.

Note that frequency-watt control only applies to resources than can generate power, including energy storage systems. It does not apply to loads. Therefore a load control system providing FFR would have no obligation or expectation to provide frequency-watt control.

System planners and operators would of course need to carefully consider the impacts of both FFR and frequency-watt control when examining the system frequency response.

The next subsection proposes a mathematically-defined method for simultaneously providing frequency-watt droop response and FFR, and subsection 7.2 presents example simulations of DER responses illustrating the application of method proposed in subsection 7.1.

\subsection{A proposed method of performing frequency-watt control simultaneously with FFR}

For the purposes of the example responses in the next subsection, it is assumed that for an underfrequency event, an FFR resource must increase its power above the pre-trigger power, $P_{\mathrm{PT}}$, by its nominated capability, $P_{\mathrm{NC}}$, as shown in equation (2).

$$
P_{F F R}=P_{P T}+P_{N C}
$$

For underfrequency events exceeding the frequency-watt deadband, $d b_{\cup F}$, the expected power, $P$, for a DER performing both frequency-watt control and FFR is shown in equation (3). At frequencies, $f$, above the FFR trigger, $59.7 \mathrm{~Hz}$, the expected power is dictated by frequency-watt control, and at frequencies below 59.7 the expected power is the greater of $P_{\mathrm{FFR}}$ and $P_{\mathrm{fw}}$ :

$$
P=\left\{\begin{array}{lr}
P_{f W} ; & 59.7<f<60-d b_{U F} \\
\max \left(P_{F F R}, P_{f W}\right) ; & f<59.7
\end{array}\right.
$$

The power dictated by frequency-watt control, $P_{\mathrm{fw}}$, would be as defined in IEEE 1547-2018,

$$
P_{f W}=\min _{f<60-d b_{U F}}\left(P_{p r e}+\frac{60-d b_{U F}-f}{60 \cdot k_{U F}}, P_{a v l}\right)
$$

where all variables are as defined in IEEE 1547-2018 clause 6.5.2.7.2. $P_{\text {pre }}$ is the pre-event power at the time the frequency first exceeded the deadband, $P_{\text {avl }}$ is the present maximum available DER power, and $k_{U F}$ is the frequency-watt droop coefficient. For most frequency events and FFR nominated capabilities, $P_{\text {FFR }}$ will be greater than $P_{\text {fw }}$ throughout the event, so the FFR response will dictate the power.

Likewise, for and overfrequency event, an FFR resource must decrease its power below the pre-trigger power, $P_{\mathrm{PT}}$, by its nominated capability, $P_{\mathrm{NC}}$, as shown in equation (5).

$$
P_{F F R}=P_{P T}-P_{N C}
$$

For overfrequency events exceeding the frequency-watt deadband, $d b_{\mathrm{OF}}$, the expected power, $P$, for a DER performing both frequency-watt control and FFR is shown in equation (6). At 
frequencies, $f$, below the FFR trigger, $60.1 \mathrm{~Hz}$, the expected power is dictated by frequency-watt control, and at frequencies above $60.1 \mathrm{~Hz}$ the expected lesser is the greater of $P_{\mathrm{FFR}}$ and $P_{\mathrm{fw}}$ :

$$
P=\left\{\begin{array}{lr}
P_{f W} ; & 60+d b_{O F}<f<60.1 \\
\min \left(P_{F F R}, P_{f W}\right) ; & 60.1<f
\end{array}\right.
$$

The power dictated by frequency-watt control, $P_{\mathrm{fw}}$, is as defined in IEEE 1547-2018: ${ }^{15}$

$$
P_{f W}=\max _{f>60+d b_{O F}}\left(P_{p r e}-\frac{f-\left(60+d b_{O F}\right)}{60 \cdot k_{O F}}, P_{\min }\right)
$$

where all variables are as defined in IEEE 1547-2018 clause 6.5.2.7.2. $P_{\min }$ is the minimum possible DER power and $k_{\mathrm{OF}}$ is the frequency-watt droop coefficient.

There is a need to define exactly when the FFR pre-trigger power, $P_{\mathrm{PT}}$, is determined. There are two likely options:

1. Define $P_{\mathrm{PT}}$ as the DER power at the moment the FFR frequency trigger is exceeded.

2. Define $P_{\mathrm{PT}}$ to be the same as $P_{\text {pre }}$ (i.e., the FFR pre-event power is defined to be equal to the DER power at the moment the frequency-watt deadband is exceeded).

Option 1 may initially seem more logical. However, under Option 1, frequency-watt will already have modified the DER power when the FFR trigger is exceeded, perhaps substantially. The effect of this will be to amplify the FFR response by an amount that depends on the details of the frequency event. This may be problematic for grid planners because the FFR response will be less predictable. In addition, DER designers would need to reserve additional power to ensure the DER will be able to meet the FFR specification under all possible frequency events, and the exact amount of additional power needed would be difficult to predict because it would depend on future grid events. This concept is illustrated via simulation of frequency events in the next subsection. For these reasons, it may be preferable to select Option 2 (i.e., define $P_{\mathrm{PT}}=$ $\left.P_{\text {pre }}\right)$. This makes for a more predicable response where both frequency-watt and FFR are based on the DER power near the start of the frequency event. In the example simulations below, Option 2 is used to determine $P_{\text {PT }}$.

\subsection{Simulation examples of simultaneous frequency-watt control and FFR}

The governor-only model of Oahu frequency dynamics pictured in Figure 34 was used to simulate the response of DERs simultaneously providing frequency-watt droop control and FFR. The simulated DERs were modified so that their frequency response followed the equations and description in section 7.1. The specific case studied was the 2019 heavy summer scenario, which produces the largest underfrequency event for that planning year. The frequency-watt control parameters for all simulations were droop ( $\left.k_{\cup F}\right)$ of $4 \%$ and deadband $\left(d b_{\cup F}\right)$ of $36 \mathrm{mHz}$. The FFR nominated capability was set to $0.2 \mathrm{pu}$, and the FFR trigger frequency was $59.7 \mathrm{~Hz}$.

\footnotetext{
${ }^{15}$ Important note: In the published version of IEEE 1547-2018, the overfrequency droop equation contains a typographical error introduced in the final typesetting. (A "-" was erroneously shown as "+".) The error was corrected in a published Errata, and the equation is shown correctly here.
} 
Figure 42 presents a simulation of DERs responding to an underfrequency event where the available DER reserve power of 0.2 pu is equal to the FFR nominated capability, as might typically be the case for a DER designed specifically to provide FFR. Figure 43 zooms in on the initial portion of the event. Both figures contain three plots:

1. Top plot: System frequency

2. Middle plot: Components of DER power change command, with net power change command in yellow

\section{Bottom plot: Simulated DER power}

The underfrequency event was triggered by the largest $\mathrm{N}-1$ contingency, a trip of the AES power plant. When the frequency drops below $59.964 \mathrm{~Hz}$ (i.e. $60-d b_{U \mathrm{~F}}$ ), the DER begins to increase power according to the underfrequency droop curve (equation 4). The frequency continues to fall, and shortly afterwards drops below $59.7 \mathrm{~Hz}$, triggering the FFR response. The DER power ramps up to its full available power, which is equal to its pre-event power plus its FFR nominated capability. While the frequency-watt command peaks much higher than the FFR command, the DER is not able to respond beyond its power limit. This is not a problem: as defined in IEEE 1547, there is no requirement to maintain headroom for frequency-watt response; the only requirement is to provide frequency-watt response if the available active power allows. The simulated DER output power is slightly smoother and slightly delayed relative to the commanded change in power because of the time dynamics of the DER inverters' power response (modeled as a first-order response with time constant of $50 \mathrm{~ms}$ ). Note that because the DER power had already increased by about 0.1 pu before the FFR response was triggered (as seen in Figure 43), if the DER were required to increase its power output by its nominated capability above its power level as measured at the moment the FFR trigger was exceeded, it would not have had the available power to meet the desired FFR response (and presumably would not have been paid for the service). This illustrates why it may be preferable to define the FFR pre-trigger power level to be equal to the frequency-watt pre-event power (i.e. Option 2 in section 7.1). 

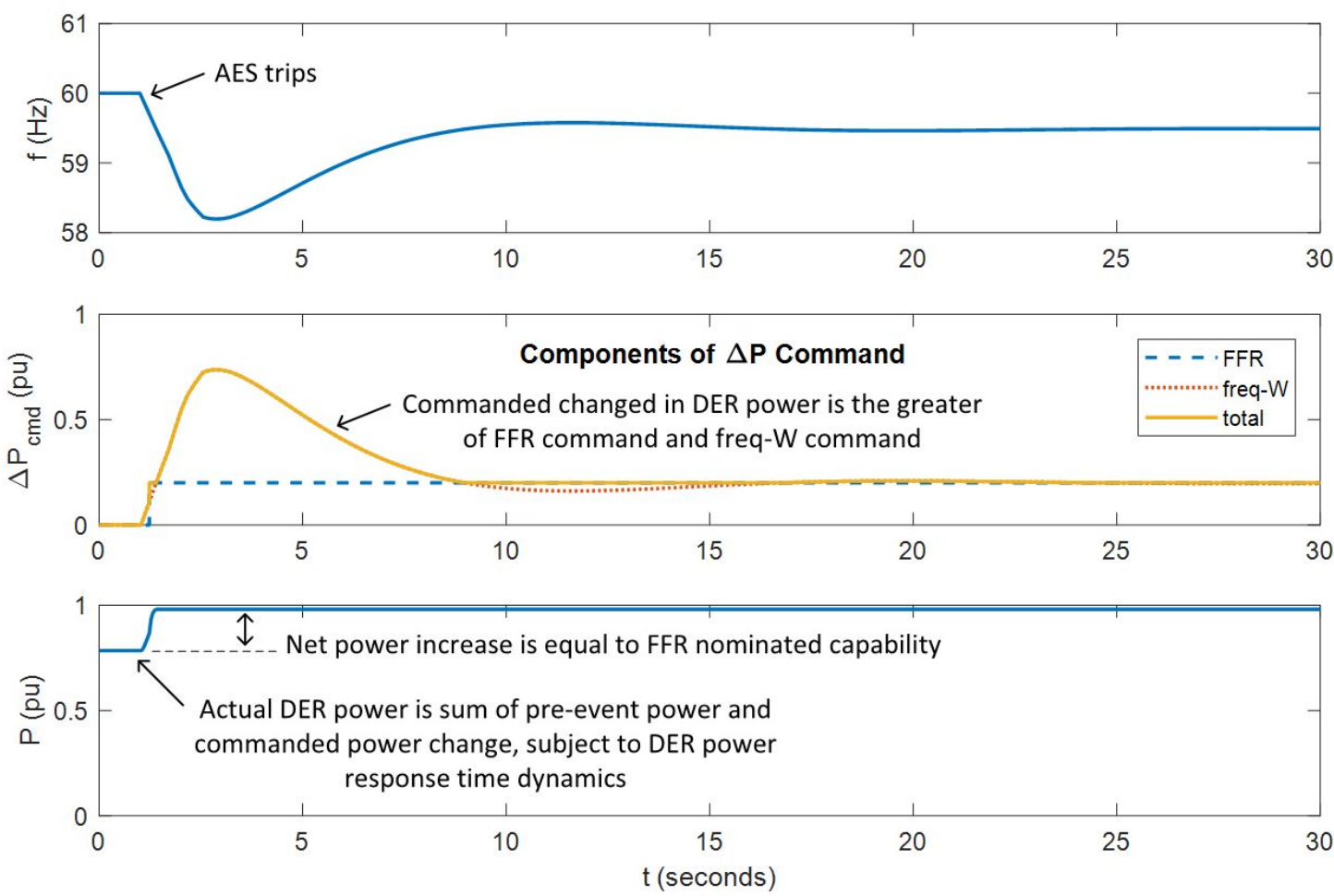

Figure 42: Simulation of DER underfrequency response while performing both frequencywatt control and FFR with power reserve equal to the FFR nominated capability.

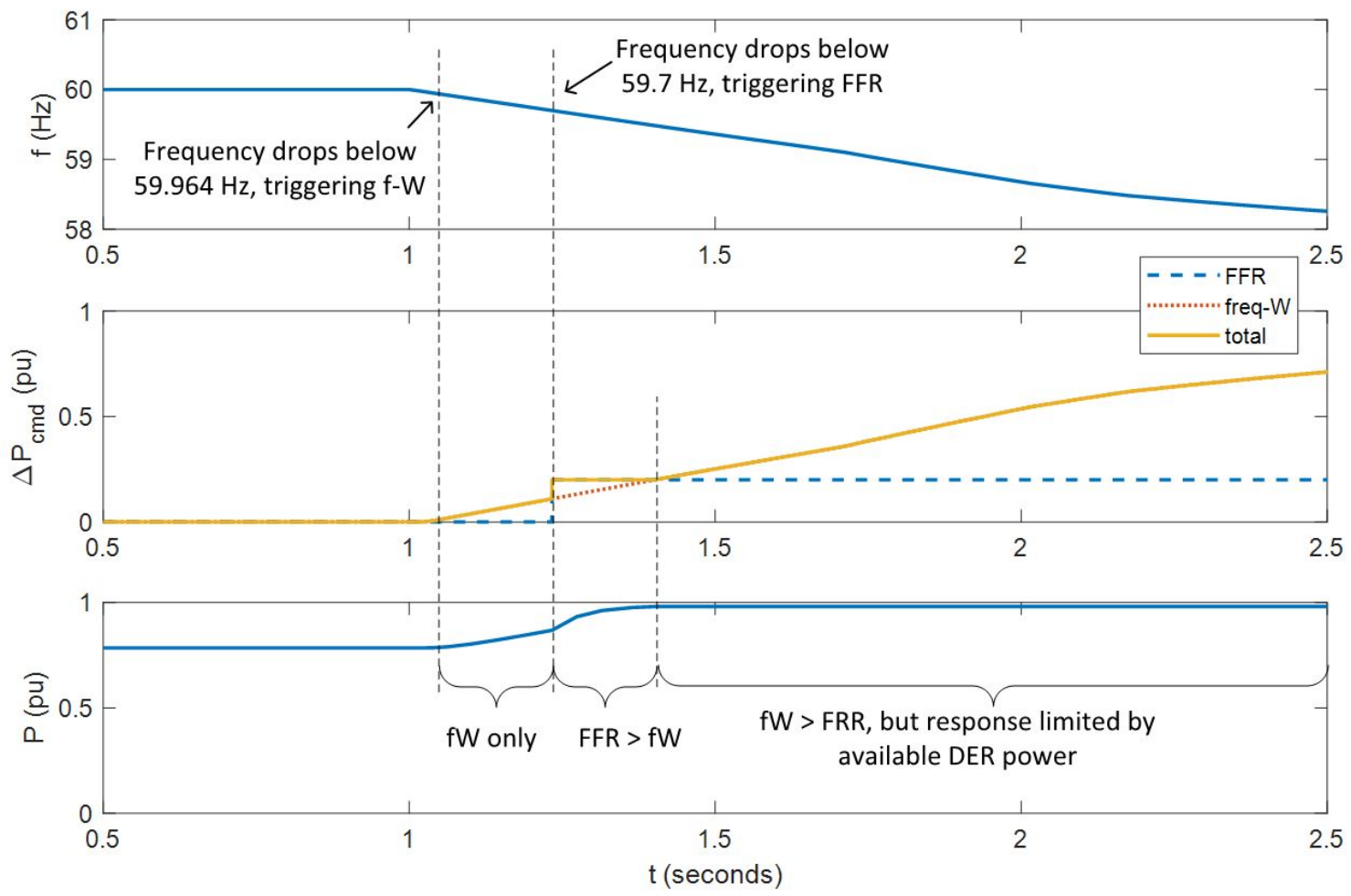

Figure 43: Close up of initial portion of Figure 42. Note that a significant portion of the power reserve is used up before the FFR response is triggered. 
Also note that if the DER was simply required to reach an absolute power equal to its nominated capability, it would have reduced its power output significantly, which is contrary to the intent of FFR and would further destabilize the system. This illustrates the importance of specifying the desired FFR response clearly.

Figure 44 presents a simulation of a DER responding to an underfrequency event in the case where the available power reserve $(0.3 \mathrm{pu})$ exceeds the FFR nominated capability $(0.2 \mathrm{pu})$, allowing some capacity for frequency-watt response even when the FFR response is fully deployed. While there may not be an economic incentive to provide this additional reserve, this scenario is useful to illustrate the full interaction between FFR and frequency-watt. A close-up of the event is shown in Figure 45. As above, this event starts with the AES plant tripping. The DER again responds initially through frequency-watt control and then through FRR. Initially the FFR command exceeds the frequency-watt command, but the frequency continues to rise, causing the frequency-watt command to be greater than the FFR command shortly after FFR is deployed. This results in an additional 0.1 pu power increase, which is maintained until the frequency drops enough to that the FFR command becomes greater, around time 9 seconds.
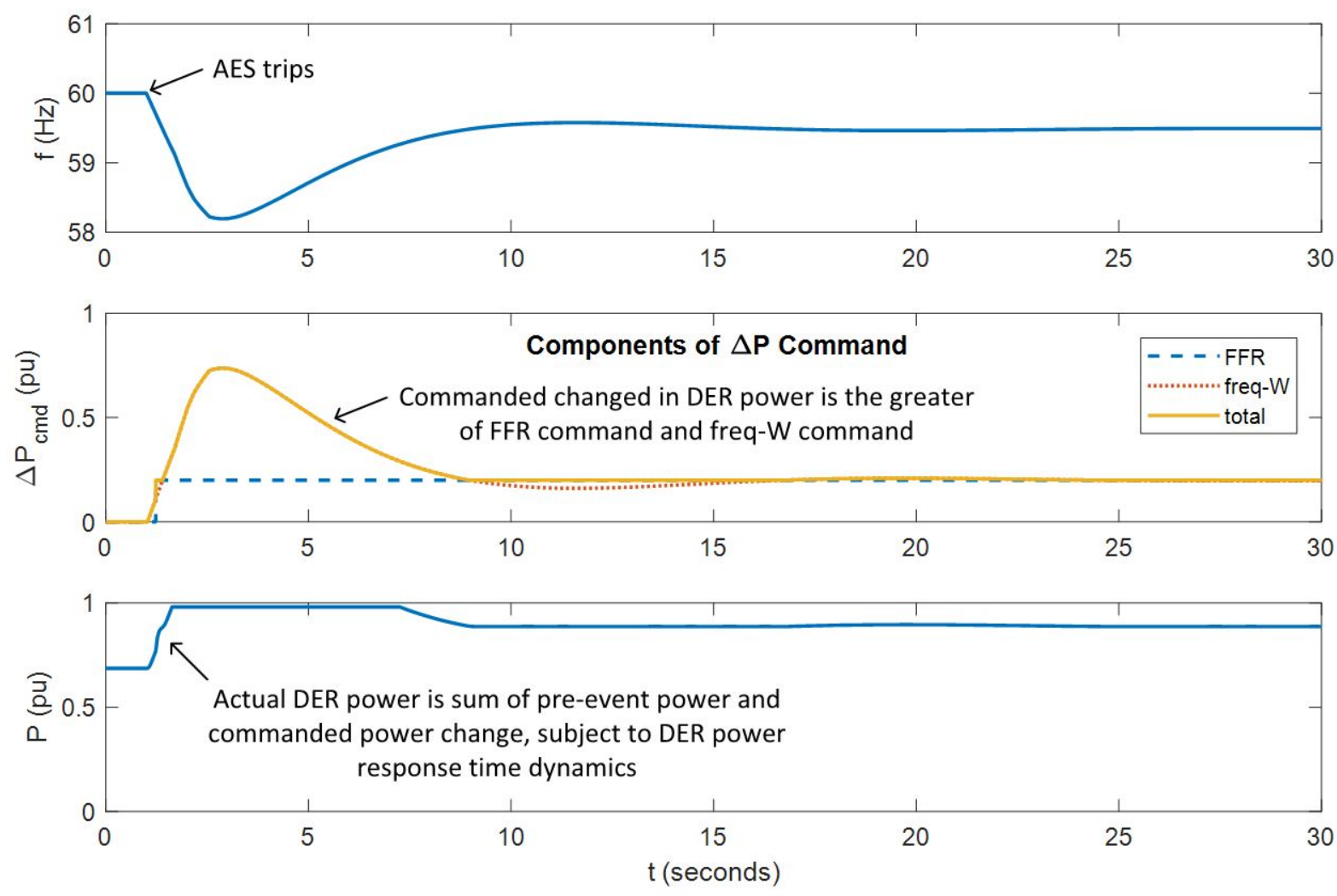

Figure 44: Simulation of DER underfrequency response while performing both frequencywatt control and FFR with power reserve exceeding the FFR nominated capability. 


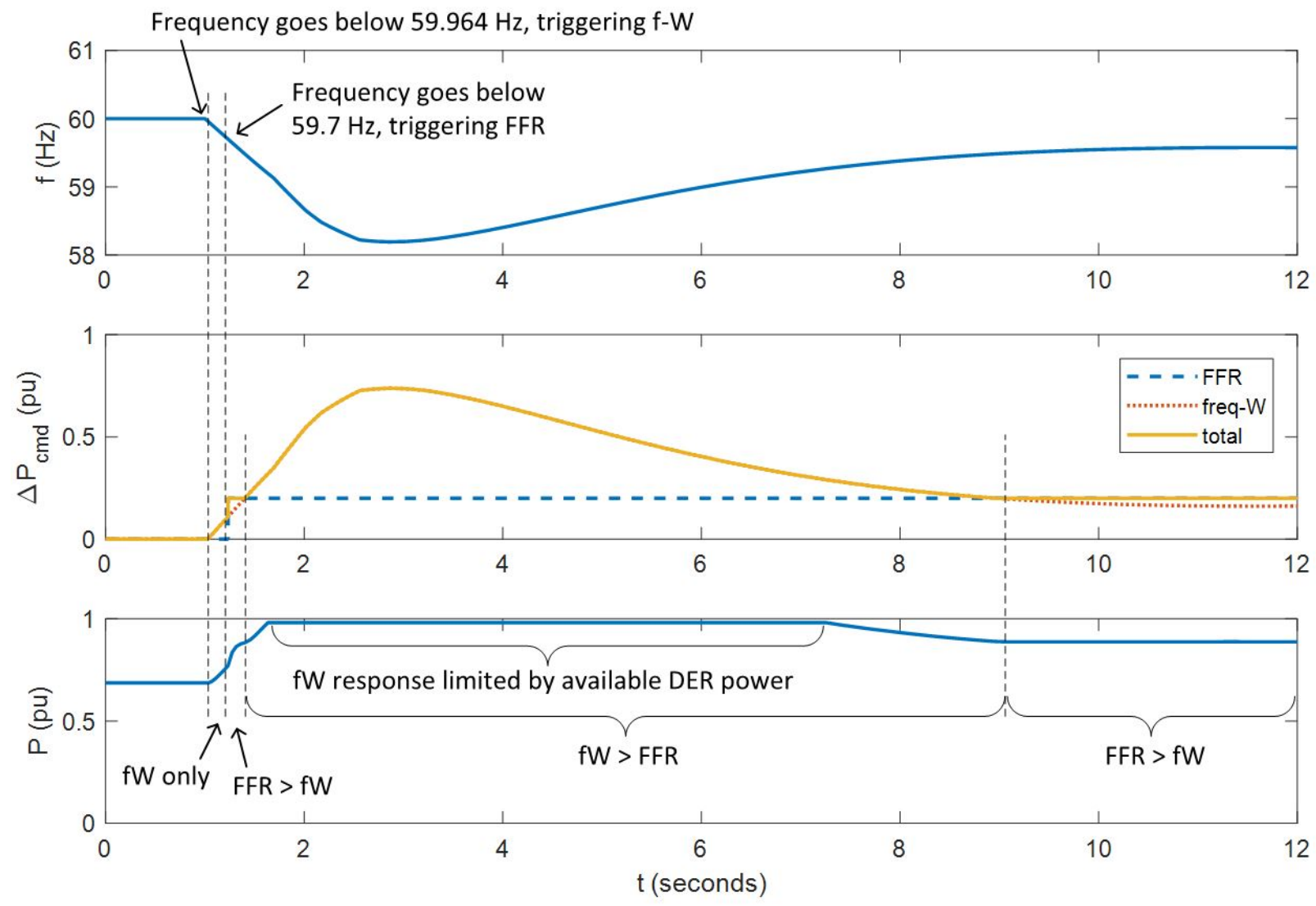

Figure 45: Close up of initial portion of Figure 44 illustrating interplay between FFR and frequency-watt control.

Note that the simulated DERs manage the transitions between regions where FFR is dominant and regions where frequency-watt is dominant relatively seamlessly simply by following equations (2) through (4), with $P_{\mathrm{PT}}$ set to be equal to $P_{\text {pre }}$ to avoid a dependency of FFR response magnitude on ROCOF. Overfrequency responses could similarly be programmed to follow equations (5) through (7), again with $P_{\mathrm{PT}}=P_{\text {pre }}$.

It is not known whether any DERs have currently been programmed to provide frequency-watt and FFR simultaneously, or what methods have been used. None of the DER inverters tested in this project had FFR capability at the time of testing. The methodology proposed here for combining FFR and frequency-watt (i.e., Option 2 in Section 7.1) could be used to allow the two functions to coexist in an achievable and predictable manner that complies with both IEEE 1547 and the Hawaiian Electric FFR tariff (assuming the authors' interpretation of the tariff to be correct). Whether this method or another method is used, it is recommended that Hawaiian Electric clearly define what method shall be used to avoid unexpected and unpredictable outcomes. All DERs are required by IEEE 1547-2018 to provide frequency-watt droop response, and defining a standard way for FFR to be coordinated with frequency-watt allows DERs to also provide FFR if desired. 


\subsection{DER Enter Service Criteria}

There is a significant potential for the high and growing levels of DERs to affect grid stability during system restoration events. Such events occur after some or all of the grid is disconnected. Currently, most DERs are programmed to return to service only after the grid voltage and frequency remain within specified enter service criteria bands for five minutes. Both the voltage and frequency criteria bands and the five-minute delay time are adjustable. The IEEE 1547-2018 ranges of adjustable settings and default values are shown in Table 10. Following an outage or trip, the DER remains offline until grid voltage and frequency remain within the enter service criteria settings for a time referred to as the enter service delay, which is adjustable between zero and 600 seconds, with a default of 300 seconds.

Table 10: Enter service criteria from IEEE 1547-2018

\begin{tabular}{|l|l|l|l|}
\hline \multicolumn{2}{|c|}{ Enter service criteria } & \multicolumn{1}{c|}{ Default settings } & \multicolumn{1}{c|}{ Ranges of allowable settings } \\
\hline \multicolumn{2}{|c|}{ Permit service } & \multicolumn{1}{c|}{ Enabled } & \multicolumn{1}{c|}{ Enabled/Disabled } \\
\hline \multirow{2}{*}{$\begin{array}{l}\text { Applicable voltage } \\
\text { within range }\end{array}$} & Minimum value & $\geq 0.917$ p.u. ${ }^{\text {a }}$ & 0.88 p.u. to 0.95 p.u. \\
\cline { 2 - 4 } & Maximum value & $\leq 1.05$ p.u. & 1.05 p.u. to 1.06 p.u. \\
\hline \multirow{2}{*}{$\begin{array}{l}\text { Frequency within } \\
\text { range }\end{array}$} & Minimum value & $\geq 59.5 \mathrm{~Hz}$ & $59.0 \mathrm{~Hz}$ to $59.9 \mathrm{~Hz}$ \\
\cline { 2 - 4 } & Maximum value & $\leq 60.1 \mathrm{~Hz}$ & $60.1 \mathrm{~Hz}$ to $61.0 \mathrm{~Hz}$ \\
\hline
\end{tabular}

aThis corresponds to the Range B of ANSI C84.1, Table 1, column for service voltage of 120-600 V.

In a future scenario where the utility can communicate with the DER, 1547-2018 provides several other options that can be used to regulate DER output during system restoration. These include the permit service setting, which can be disabled until the system is ready for the DERs to interconnect, and the limit active power setting, which could be increased gradually or incrementally to allow DERs to come online in a manner that is compatible with system restoration needs. Both of these options would offer improved controllability and should certainly be considered once the utility is able to communicate with many DERs.

However, at present the vast majority of DERs have no communications, so currently the best way of ensuring DERs do not reconnect en masse to a grid that is not yet stable is likely to use relatively tight enter service criteria ranges. The default 1547-2018 values for voltage are acceptable, as is the default value for overfrequency. However, in the case of Hawaii the default value for underfrequency, $59.5 \mathrm{~Hz}$, could be problematic as it could result in a large number of DERs reconnecting simultaneous while the system is still not stable. While the connection of additional generation will increase frequency, it could potentially come online too quickly or drop rapidly due to cloud transients while grid operators are still working to stabilize the grid, disrupting the recovery effort.

\subsection{Impact of Enter Service Criteria on DER Energy Production}

DER industry stakeholders have expressed concerns about the impact of enter service criteria frequency settings on DER energy production. To address this concern, a detailed analysis of the impact of enter service criteria was performed using recorded frequency data from Oahu, Maui, and Hawaii islands. NREL analyzed a full year (2017) of two-second resolution frequency data and identified all frequency excursions outside Hawaiian Electric's proposed frequency 
enter service criteria of 59.9 to $60.1 \mathrm{~Hz}$. For each frequency excursion, the duration and time of day was identified, and the impact on DER energy production was estimated.

PV systems typically go into a sleep mode overnight to reduce energy consumption, so they reconnect to the grid every morning. In order to reconnect, they must detect a five-minute window during which frequency is within the enter service criteria range. Field data collected by Hawaiian Electric and NREL indicate that energy storage DERs typically remain connected to the grid 24 hours per day (even if not charging or discharging), so energy storage DERs do not need to regularly reconnect. All DERs do need to reconnect following any trip events caused by utility outages or major grid disturbances outside the voltage and frequency trip settings. Such events tend to occur much less than once per day, especially now that Hawaii implemented voltage and frequency ride-through requirements, so the dominant impact of DER reconnection is during the morning $\mathrm{PV}$ reconnection period.

To facilitate analyzing the impact of enter service criteria on DER energy production, NREL defined the term unable-to-reconnect window as a continuous time window where an inverter that is offline is barred from reconnecting due to grid frequency being outside the enter service criteria range, illustrated in Figure 46. An unable-to-reconnect window includes the duration of the frequency excursion and the five minutes following the end of the event. If another frequency excursion occurs before five minutes have passed, the unable-to-reconnect window is lengthened accordingly, as illustrated in Figure 46. Note that DERs that are online are not affected during unable-to-reconnect windows; they only affect reconnection of offline DERs.
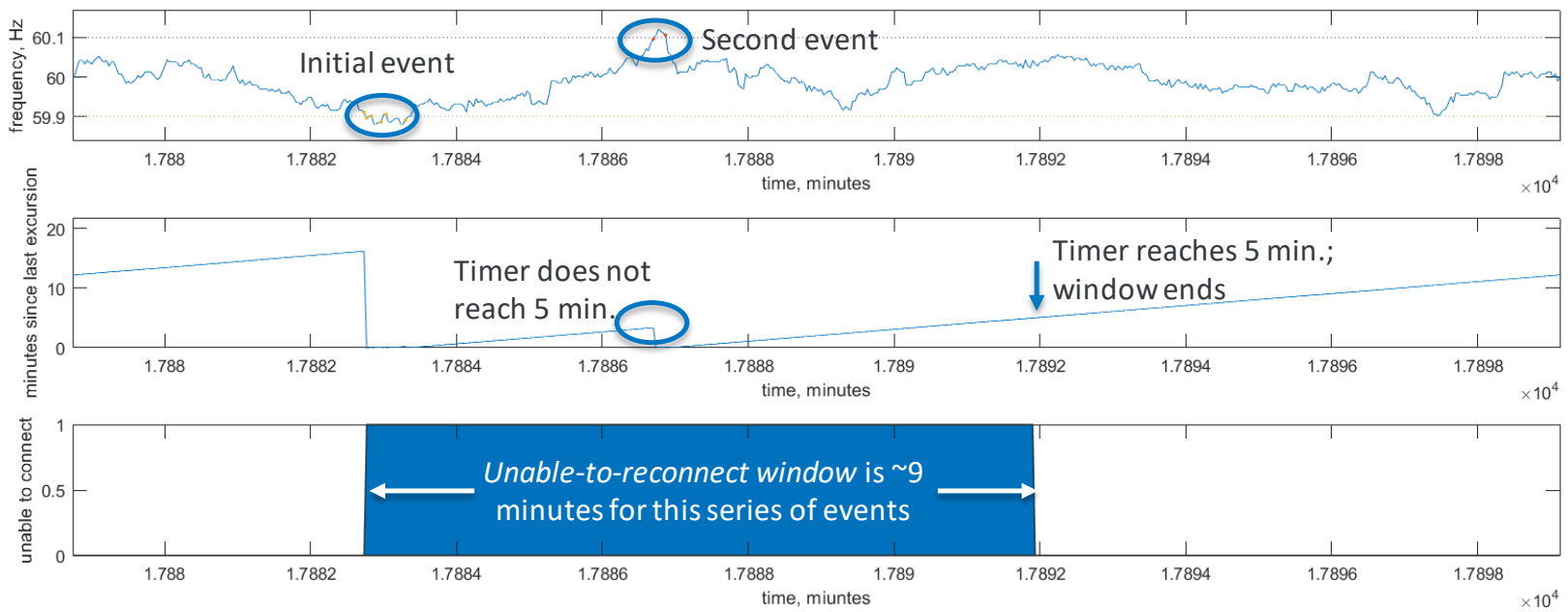

Figure 46: Illustration of an unable-to-reconnect window showing recorded grid frequency, inverter reconnection timer, and duration of unable-to-reconnect window.

Figure 47 shows an example of the unable-to-reconnect windows during a typical month of frequency data (March 2017, Hawaii Island). The unable-to-reconnect windows appear as vertical lines because they are very short relative to the duration of the plot. The longest unableto-reconnect window is about 13 minutes. 

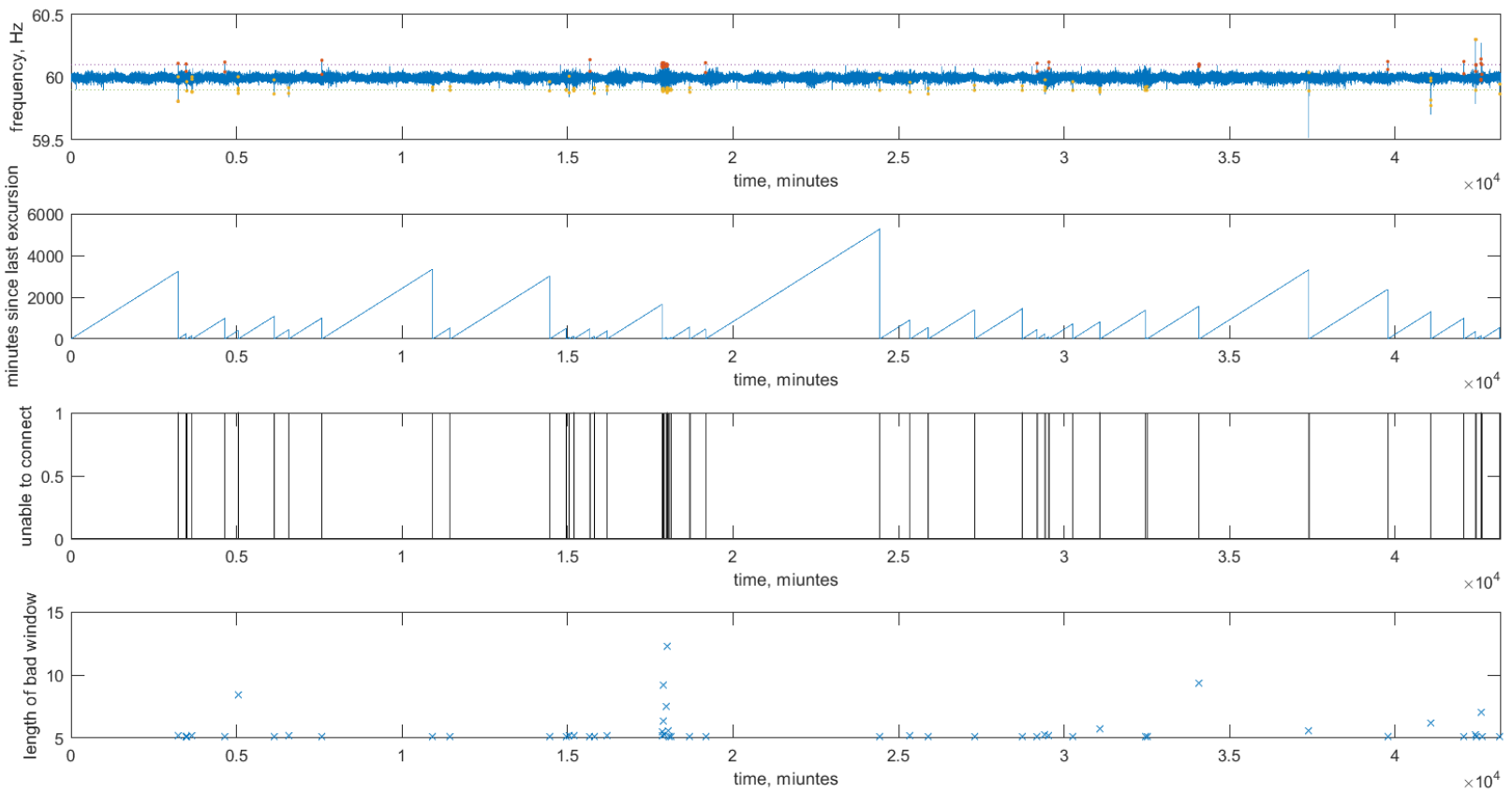

Figure 47: Unable-to-reconnect windows during a typical month (March 2017, Hawaii Island).

Figure 48 shows the unable-to-reconnect windows during the worst month (Maui during June 2017). This month contains many more unable-to-reconnect windows, and the windows tend to be longer, with the longest window approaching 40 minutes. Recall that this window has no effect on the vast majority of DERs; it only effects a DER that is offline and attempting to reconnect at the time. While at a glance it may appear from the thick black vertical lines that some of the unable-to-reconnect windows are longer than 40 minutes, each of the thicker lines represents a series of unable-to-reconnect windows with opportunities between windows for inverters to reconnect, as illustrated in Figure 49. 

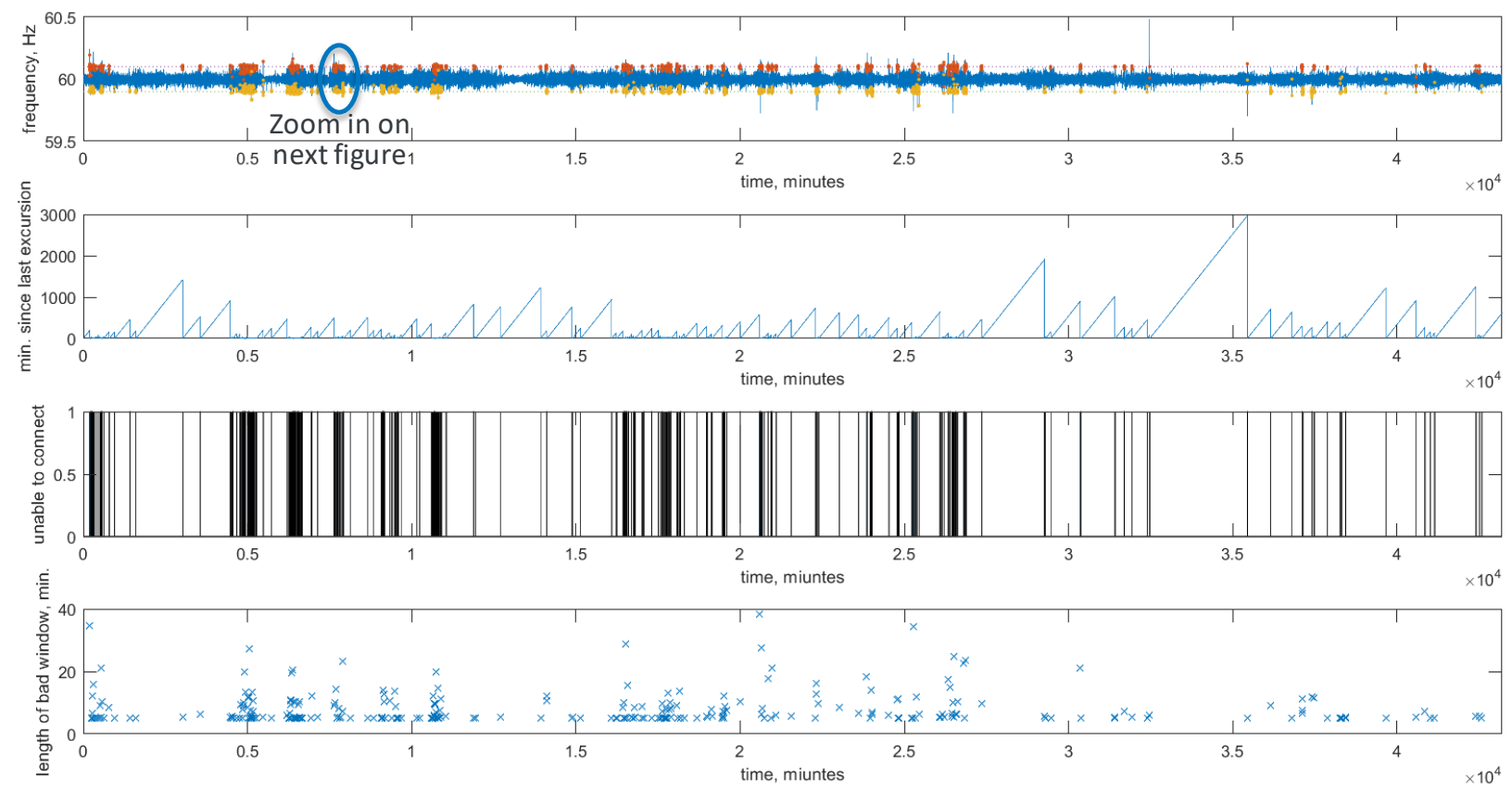

Figure 48: Unable-to-reconnect windows during the worst-case month (June 2017, Maui).
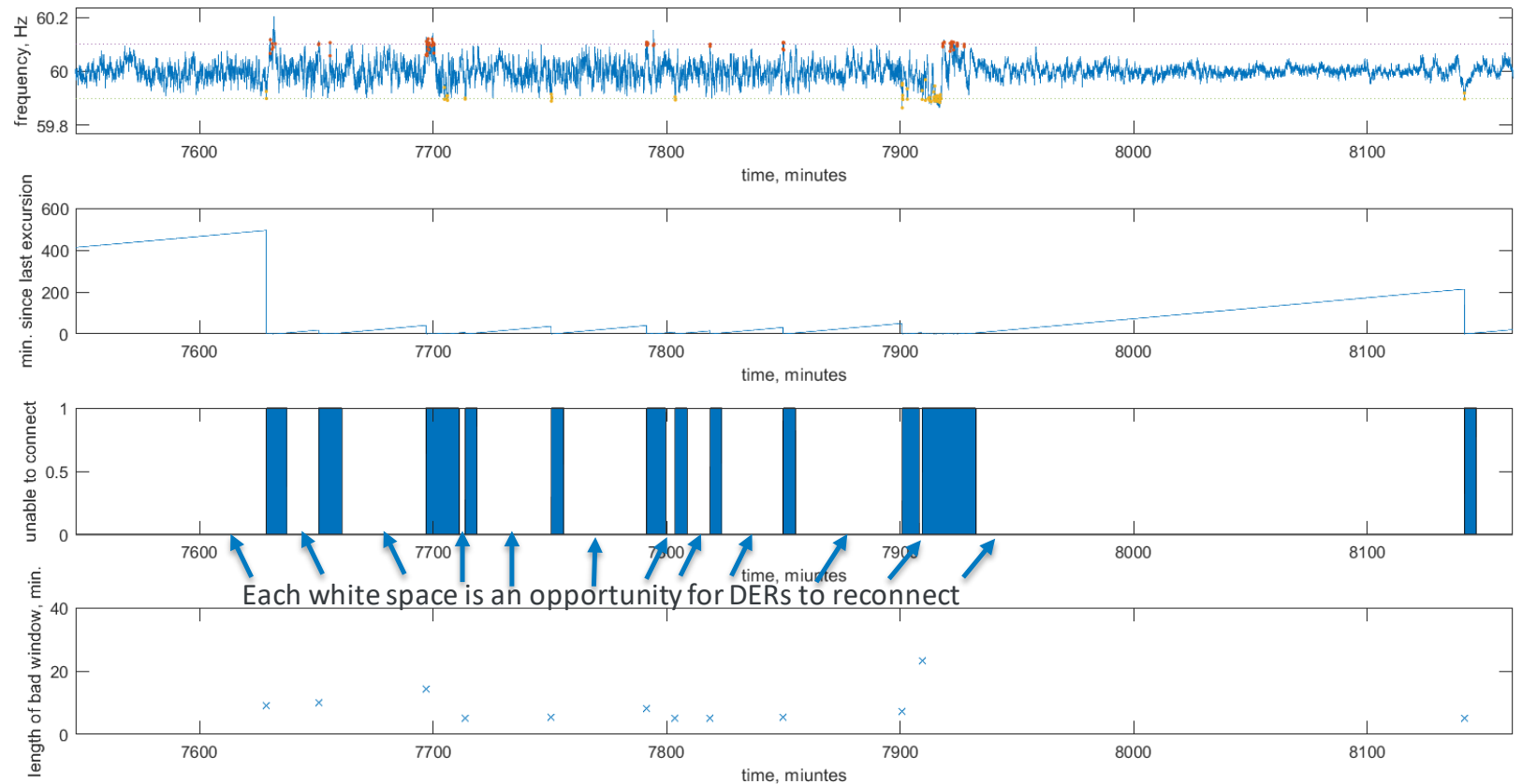

Figure 49: A closer view of unable-to-reconnect windows for the circled period in Figure 48 showing that what appears to be a single very long unable-to-reconnect window is actually several windows with opportunities in between for any offline DERs to reconnect.

Table 11, Table 12, and Table 13 summarize the number of unable-to-reconnect windows, the window lengths, and the percentage time during which an offline DER would be unable to reconnect for each month for Oahu, Maui, and Hawaii Island, respectively. Note that the 
percentage of time during which an offline DER would be unable to reconnect is not equivalent to "percentage of energy curtailed". A DER that is online during an unable-to-reconnect window will remain online, so curtailment will be much smaller than these numbers, as estimated in a later portion of this section. Overall, the amount of time when DERs would be unable to reconnect using the proposed enter service criteria is very small. There was a clear trend of higher levels of frequency excursions on Maui between January and June 2017. This is attributed to wind plants not obeying curtailment commands and violating ramps rates, and to battery plants that were non-responsive during this time. The longest unable-to-reconnect window of 54 minutes occurred during that time period.

Table 11: Unable-to-reconnect window data, Oahu, 2017

\begin{tabular}{|c|c|c|c|c|}
\hline & $\begin{array}{c}\text { Number of } \\
\text { windows }\end{array}$ & $\begin{array}{l}\text { Average } \\
\text { length of } \\
\text { window } \\
\text { (minutes) }\end{array}$ & $\begin{array}{l}\text { Longest } \\
\text { window } \\
\text { (minutes) }\end{array}$ & $\begin{array}{l}\text { Percent of } \\
\text { time unable- } \\
\text { to-reconnect }\end{array}$ \\
\hline Jan & 44 & 6.2 & 10.3 & $0.61 \%$ \\
\hline Feb & 40 & 6.7 & 17.3 & $0.66 \%$ \\
\hline Mar & 27 & 6.6 & 13.2 & $0.40 \%$ \\
\hline Apr & 30 & 6.3 & 10.2 & $0.44 \%$ \\
\hline May & 25 & 6.4 & 16.0 & $0.36 \%$ \\
\hline Jun & 25 & 6.1 & 14.8 & $0.35 \%$ \\
\hline Jul & 4 & 5.2 & 5.3 & $0.05 \%$ \\
\hline Aug & 17 & 5.4 & 7.4 & $0.20 \%$ \\
\hline Sep & 7 & 5.2 & 5.8 & $0.08 \%$ \\
\hline Oct & 37 & 5.7 & 11.3 & $0.47 \%$ \\
\hline Nov & 38 & 5.6 & 9.7 & $0.49 \%$ \\
\hline Dec & 22 & 6.7 & 11.2 & $0.33 \%$ \\
\hline Totals & 316 & 6.0 & 17.3 & $0.37 \%$ \\
\hline
\end{tabular}


Table 12: Unable-to-reconnect window data, Maui, 2017

\begin{tabular}{|c|c|c|c|c|}
\hline & $\begin{array}{l}\text { Number of } \\
\text { windows }\end{array}$ & $\begin{array}{l}\text { Average } \\
\text { length of } \\
\text { window } \\
\text { (minutes) }\end{array}$ & $\begin{array}{l}\text { Longest } \\
\text { window } \\
\text { (minutes) }\end{array}$ & $\begin{array}{l}\text { Percent of } \\
\text { time unable- } \\
\text { to-reconnect }\end{array}$ \\
\hline Jan & 151 & 7.2 & 35.7 & $2.41 \%$ \\
\hline Feb & 185 & 7.5 & 54.5 & $3.44 \%$ \\
\hline Mar & 133 & 7.5 & 38.1 & $2.21 \%$ \\
\hline Apr & 188 & 6.5 & 18.0 & $2.81 \%$ \\
\hline May & 255 & 7.4 & 48.5 & $4.21 \%$ \\
\hline Jun & 291 & 8.1 & 38.4 & $5.44 \%$ \\
\hline Jul & 42 & 6.1 & 17.5 & $0.57 \%$ \\
\hline Aug & 79 & 8.0 & 21.7 & $1.40 \%$ \\
\hline Sep & 25 & 6.0 & 9.6 & $0.35 \%$ \\
\hline Oct & 63 & 7.9 & 28.2 & $1.10 \%$ \\
\hline Nov & 78 & 13.6 & 6.8 & $1.22 \%$ \\
\hline Dec & 80 & 7.3 & 26.4 & $1.29 \%$ \\
\hline Totals & 1570 & 7.7 & 54.5 & $2.20 \%$ \\
\hline
\end{tabular}

Table 13: Unable-to-reconnect window data, Hawaii Island, 2017

\begin{tabular}{|c|c|c|c|c|}
\hline & $\begin{array}{c}\text { Number of } \\
\text { windows }\end{array}$ & $\begin{array}{l}\text { Average } \\
\text { length of } \\
\text { window } \\
\text { (minutes) }\end{array}$ & $\begin{array}{l}\text { Longest } \\
\text { window } \\
\text { (minutes) }\end{array}$ & $\begin{array}{l}\text { Percent of } \\
\text { time unable- } \\
\text { to-reconnect }\end{array}$ \\
\hline Jan & 130 & 5.0 & 9.0 & $1.56 \%$ \\
\hline Feb & 193 & 5.6 & 10.2 & $2.68 \%$ \\
\hline Mar & 95 & 5.8 & 11.3 & $1.19 \%$ \\
\hline Apr & 52 & 5.7 & 12.2 & $0.68 \%$ \\
\hline May & 51 & 5.6 & 12.7 & $0.63 \%$ \\
\hline Jun & 39 & 6.8 & 39.0 & $0.61 \%$ \\
\hline Jul & 43 & 5.2 & 5.4 & $0.49 \%$ \\
\hline Aug & 26 & 5.2 & 6.4 & $0.30 \%$ \\
\hline Sep & 46 & 5.4 & 8.9 & $0.57 \%$ \\
\hline Oct & 186 & 5.6 & 16.5 & $2.26 \%$ \\
\hline Nov & 96 & 5.5 & 15.9 & $1.18 \%$ \\
\hline Dec & 101 & 5.4 & 11.5 & $1.21 \%$ \\
\hline Totals & 1058 & 5.6 & 39.0 & $1.11 \%$ \\
\hline
\end{tabular}

Figure 50, Figure 51, and Figure 52 show the percentage of time when an offline DER would be unable to reconnect under the proposed enter service criteria for Oahu, Maui, and Hawaii Island, respectively. The PV morning startup hours of 6 am to 8 am are highlighted in yellow. For all three islands, frequency excursions were more prevalent during the $8 \mathrm{am}$ to $4 \mathrm{pm}$ hours, when PV production is already online and not affected. The prevalence of frequency excursions 
during the $10 \mathrm{pm}$ to $1 \mathrm{am}$ period on Hawaii Island, especially during the October to February time frame, is attributed to wind power ramping.

Percent time unable to reconnect by hour of day

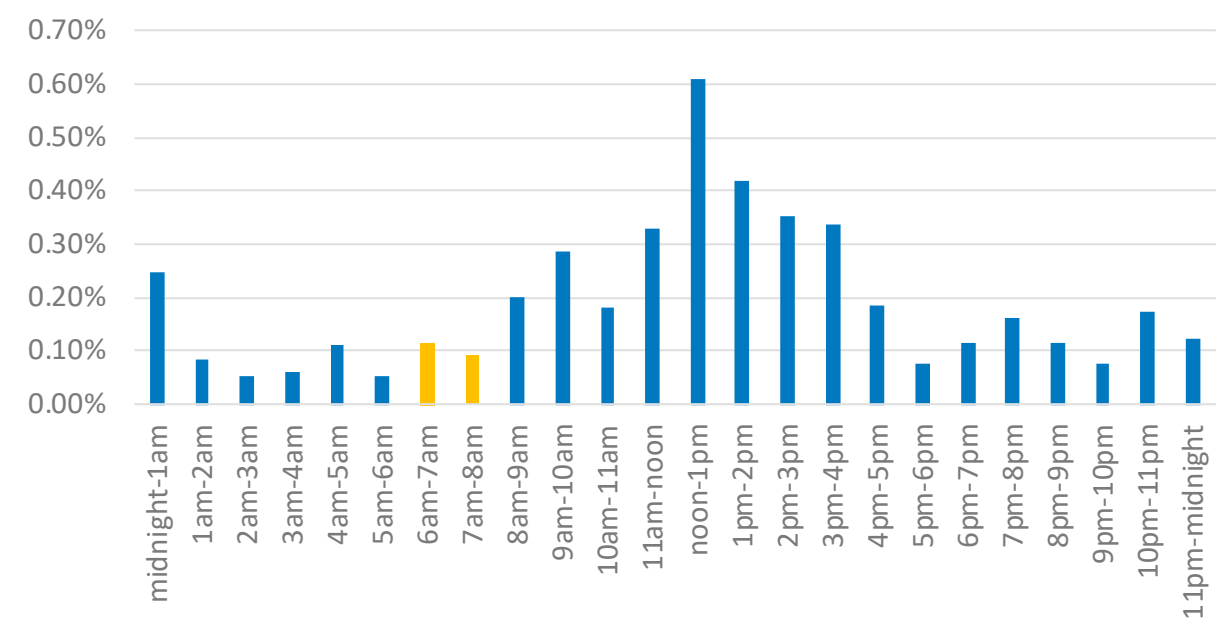

Figure 50: Percentages of time covered by unable-to-reconnect windows by hour of day for Oahu.

Percent time unable to reconnect by hour of day

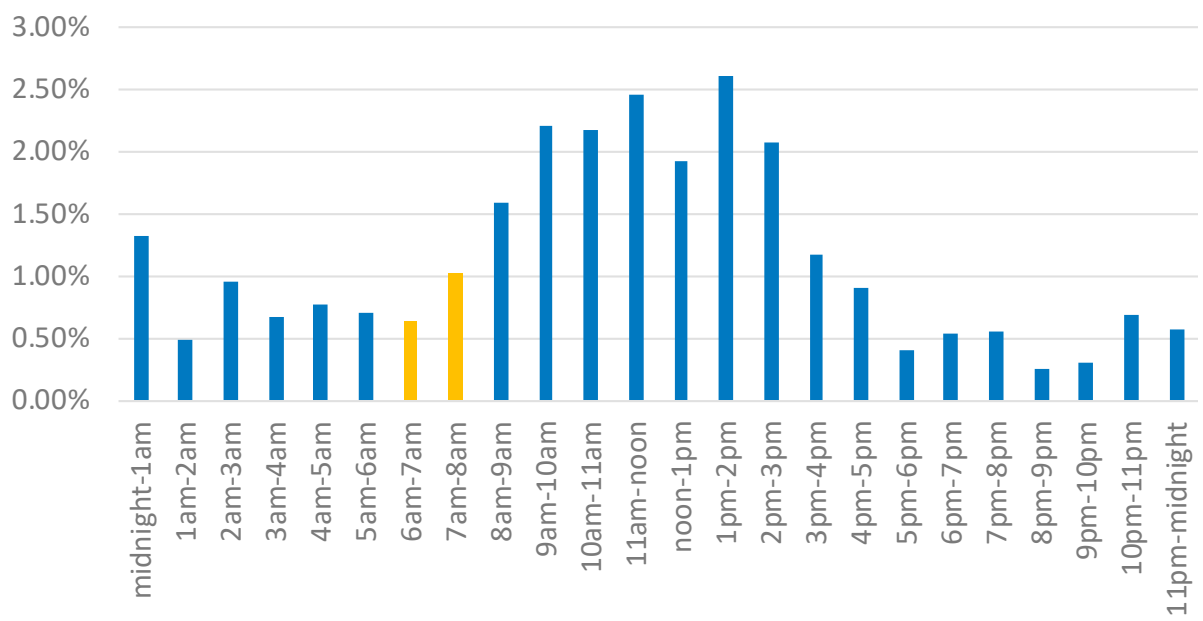

Figure 51: Percentages of time covered by unable-to-reconnect windows by hour of day for Maui. 


\section{Percent time unable to reconnect by hour of day}

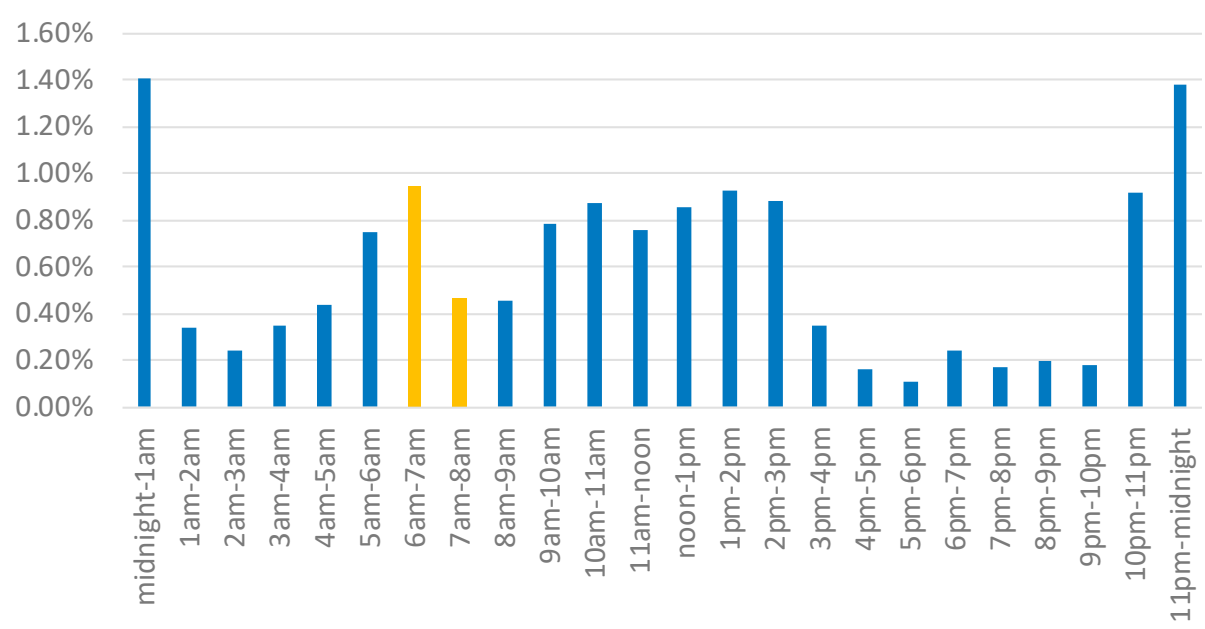

Figure 52: Percentages of time covered by unable-to-reconnect windows by hour of day for Hawaii Island.

To estimate the impact of the proposed 59.9 to $60.1 \mathrm{~Hz}$ enter service criteria on DER energy production, we first calculate the estimated number of minutes per month that a typical DER would not be able to produce energy:

$$
\begin{aligned}
& \text { Lost production minutes per month } \\
& \qquad \begin{array}{l}
=\left(\frac{\text { Reconnections }}{\text { month }}\right) *(\text { Fraction of time unable to reconnect }) \\
*(\text { Average length of unable to reconnect window })
\end{array}
\end{aligned}
$$

We can then calculate the estimated lost energy production as:

$$
\text { Lost energy production }=\frac{\text { Lost production minutes per month }}{(\text { Total minutes per month }) *(\text { DER capacity factor })}
$$

We use a typical PV capacity factor of 0.2 . The average number of reconnections per day for PV systems is expected to be slightly above one including one reconnection per morning plus an occasional reconnection following a major grid disturbance that caused a trip. We calculate the lost energy production assuming 30 reconnections per month and again using 90 reconnections per month (three reconnections per day, as potential extreme case). The results are shown in Table 14, Table 15, and Table 16. Even in the extreme case of three reconnections per day, the estimated lost annual energy production is less than $0.2 \%$ of the expected annual production, i.e. a negligible amount. These estimates do not account for the fact that most frequency excursions occur outside the morning PV startup window, as shown above, so the actual impact expected to be lower.

These tables focus on PV DERs. Battery systems typically to remain connected even when not producing or absorbing power, so battery systems only need to reconnect following a major grid disturbance or manual disconnection. Therefore the average number of reconnections per day is expected to be much less than one for battery DERs. For these reasons, downtime for battery DERs due to enter service criteria is expected to be lower than that shown in Table 14, Table 15, and Table 16. 
Table 14: Lost PV energy production due to proposed enter service criteria, Oahu, 2017

\begin{tabular}{|c|c|c|c|c|c|c|}
\hline & \begin{tabular}{|c|} 
Reconnects \\
per day
\end{tabular} & $\begin{array}{c}\text { Lost } \\
\text { production } \\
\text { minutes } \\
\text { per month }\end{array}$ & $\begin{array}{c}\text { Lost energy } \\
\text { production } \\
\text { (\% of } \\
\text { expected) }\end{array}$ & $\begin{array}{c}\text { Reconnects } \\
\text { per day }\end{array}$ & $\begin{array}{c}\text { Lost } \\
\text { production } \\
\text { minutes } \\
\text { per month }\end{array}$ & $\begin{array}{c}\text { Lost energy } \\
\text { production } \\
\text { (\% of } \\
\text { expected) }\end{array}$ \\
\hline Jan & 1 & 1.1 & $0.01 \%$ & 3 & 3.4 & $0.04 \%$ \\
\hline Feb & 1 & 1.3 & $0.02 \%$ & 3 & 4.0 & $0.05 \%$ \\
\hline Mar & 1 & 0.8 & $0.01 \%$ & 3 & 2.4 & $0.03 \%$ \\
\hline Apr & 1 & 0.8 & $0.01 \%$ & 3 & 2.5 & $0.03 \%$ \\
\hline May & 1 & 0.7 & $0.01 \%$ & 3 & 2.1 & $0.02 \%$ \\
\hline Jun & 1 & 0.6 & $0.01 \%$ & 3 & 1.9 & $0.02 \%$ \\
\hline Jul & 1 & 0.1 & $0.00 \%$ & 3 & 0.2 & $0.00 \%$ \\
\hline Aug & 1 & 0.3 & $0.00 \%$ & 3 & 1.0 & $0.01 \%$ \\
\hline Sep & 1 & 0.1 & $0.00 \%$ & 3 & 0.4 & $0.00 \%$ \\
\hline Oct & 1 & 0.8 & $0.01 \%$ & 3 & 2.4 & $0.03 \%$ \\
\hline Nov & 1 & 0.8 & $0.01 \%$ & 3 & 2.5 & $0.03 \%$ \\
\hline Dec & 1 & 0.7 & $0.01 \%$ & 3 & 2.0 & $0.02 \%$ \\
\hline Average & 1 & 0.69 & $0.01 \%$ & 3 & 2.06 & $0.02 \%$ \\
\hline
\end{tabular}


Table 15: Lost PV energy production due to proposed enter service criteria, Maui, 2017

\begin{tabular}{|c|c|c|c|c|c|c|}
\hline & $\begin{array}{c}\text { Reconnects } \\
\text { per day }\end{array}$ & $\begin{array}{c}\text { Lost } \\
\text { production } \\
\text { minutes } \\
\text { per month }\end{array}$ & $\begin{array}{c}\text { Lost energy } \\
\text { production } \\
\text { (\% of } \\
\text { expected) }\end{array}$ & $\begin{array}{c}\text { Reconnects } \\
\text { per day }\end{array}$ & $\begin{array}{c}\text { Lost } \\
\text { production } \\
\text { minutes } \\
\text { per month }\end{array}$ & $\begin{array}{c}\text { Lost energy } \\
\text { production } \\
\text { (\% of } \\
\text { expected) }\end{array}$ \\
\hline Jan & 1 & 5.2 & $0.06 \%$ & 3 & 15.5 & $0.18 \%$ \\
\hline Feb & 1 & 7.8 & $0.09 \%$ & 3 & 23.3 & $0.27 \%$ \\
\hline Mar & 1 & 4.9 & $0.06 \%$ & 3 & 14.8 & $0.17 \%$ \\
\hline Apr & 1 & 5.5 & $0.06 \%$ & 3 & 16.4 & $0.19 \%$ \\
\hline May & 1 & 9.4 & $0.11 \%$ & 3 & 28.1 & $0.33 \%$ \\
\hline Jun & 1 & 13.2 & $0.15 \%$ & 3 & 39.7 & $0.46 \%$ \\
\hline Jul & 1 & 1.0 & $0.01 \%$ & 3 & 3.1 & $0.04 \%$ \\
\hline Aug & 1 & 3.3 & $0.04 \%$ & 3 & 10.0 & $0.12 \%$ \\
\hline Sep & 1 & 0.6 & $0.01 \%$ & 3 & 1.9 & $0.02 \%$ \\
\hline Oct & 1 & 2.6 & $0.03 \%$ & 3 & 7.8 & $0.09 \%$ \\
\hline Nov & 1 & 5.0 & $0.06 \%$ & 3 & 14.9 & $0.17 \%$ \\
\hline Dec & 1 & 2.8 & $0.03 \%$ & 3 & 8.4 & $0.10 \%$ \\
\hline Average & 1 & 5.11 & $0.06 \%$ & 3 & 15.34 & $0.18 \%$ \\
\hline
\end{tabular}


Table 16: Lost PV energy production due to proposed enter service criteria, Hawaii Island, 2017

\begin{tabular}{|c|c|c|c|c|c|c|}
\hline & \begin{tabular}{|c} 
Reconnects \\
per day
\end{tabular} & $\begin{array}{c}\text { Lost } \\
\text { production } \\
\text { minutes } \\
\text { per month }\end{array}$ & $\begin{array}{c}\text { Lost energy } \\
\text { production } \\
\text { (\% of } \\
\text { expected) }\end{array}$ & $\begin{array}{c}\text { Reconnects } \\
\text { per day }\end{array}$ & $\begin{array}{c}\text { Lost } \\
\text { production } \\
\text { minutes } \\
\text { per month }\end{array}$ & $\begin{array}{c}\text { Lost energy } \\
\text { production } \\
\text { (\% of } \\
\text { expected) }\end{array}$ \\
\hline Jan & 1 & 2.4 & $0.03 \%$ & 3 & 7.1 & $0.08 \%$ \\
\hline Feb & 1 & 4.5 & $0.05 \%$ & 3 & 13.6 & $0.16 \%$ \\
\hline Mar & 1 & 2.1 & $0.02 \%$ & 3 & 6.2 & $0.07 \%$ \\
\hline Apr & 1 & 1.2 & $0.01 \%$ & 3 & 3.5 & $0.04 \%$ \\
\hline May & 1 & 1.1 & $0.01 \%$ & 3 & 3.2 & $0.04 \%$ \\
\hline Jun & 1 & 1.2 & $0.01 \%$ & 3 & 3.7 & $0.04 \%$ \\
\hline Jul & 1 & 0.8 & $0.01 \%$ & 3 & 2.3 & $0.03 \%$ \\
\hline Aug & 1 & 0.5 & $0.01 \%$ & 3 & 1.4 & $0.02 \%$ \\
\hline Sep & 1 & 0.9 & $0.01 \%$ & 3 & 2.8 & $0.03 \%$ \\
\hline Oct & 1 & 3.8 & $0.04 \%$ & 3 & 11.5 & $0.13 \%$ \\
\hline Nov & 1 & 2.0 & $0.02 \%$ & 3 & 5.9 & $0.07 \%$ \\
\hline Dec & 1 & 1.9 & $0.02 \%$ & 3 & 5.8 & $0.07 \%$ \\
\hline Average & 1 & 1.86 & $0.02 \%$ & 3 & 5.57 & $0.06 \%$ \\
\hline
\end{tabular}

In summary, the impact of the proposed enter service criteria on DER energy production is expected to be negligible even for extreme cases. Even assuming a future grid scenario with a tenfold increase in frequency deviations, the impact would still be very small.

\subsection{Impact of Enter Service Criteria on System Restoration}

The primary need for tighter enter service criteria in a system with very high levels of DERs is during system restoration following a blackout of most or all of the system. In such a scenario, grid operators typically energize small portions of the system at a time, starting with black start resources in the case of a complete blackout. As grid operators attempt to re-energize the system by gradually adding generation and load, the system is much more vulnerable to disturbances than during normal operations because it has lower inertia and may lack normal levels of reserve. In a system with very high levels of DERs embedded in load, todays DERs have no way of knowing that the system is in a precarious state and hence will begin to come online when the circuits to which they are connected are energized. There is a potential that too many DERs will come online and destabilize the system restoration process, making it more difficult to restore power. Such a scenario is difficult to simulate because conventional planning tools are not designed for it. It would require a significant research effort to build up a system restoration model with sufficient detail to accurately simulate the effects of uncontrolled DERs on system restoration, but it is expected that the effect of uncontrolled DERs would be destabilizing in at least some scenarios.

It may be possible in the future to enable DERs to actively participate in black start and system restoration; in fact there are active research projects investigating this. However, today's DERs do not have this capability, so it is recommended that tight enter service criteria be implemented 
for the time being to avoid a scenario where uncontrolled DER reconnection destabilizes a system while it is being restored, potentially prolonging a major outage.

\subsection{Conclusions and Recommendations}

\subsection{Summary of Findings}

As confirmed by the hardware tests shown in this report, most currently-available residentialscale PV inverters can perform frequency-watt control, and can do so reliably. This result is consistent with other recent test publications [28], [34], [35]. However, the exact form and timedomain response of the frequency-watt function varies between inverters models. At the time of testing, most battery inverters did not have additional frequency response capability beyond what was available from PV inverters: some had no autonomous frequency response capability, and others responded only to overfrequency events. However, this is expected to change as inverters are updated to comply with IEEE 1547-2018.

The tests and simulations in this report have shown that the basic $\mathrm{PV}$ inverter frequency-watt function can be beneficial for stabilizing overfrequency events, and could also be beneficial for stabilizing underfrequency events if sufficient energy storage were installed, of if PV were operated with reserve. No adverse effects on the distribution system from frequency-watt control were observed. Available firmware for most residential-scale PV and storage inverters did not allow for this at the time of testing but is expected to do so in the future, and IEEE 1547-2018 requires it. The simulations and tests focused on the Oahu power system.

Very steep droop slopes, i.e. steeper than $3 \%$, were found to cause increased frequency oscillations in some cases, while droops slopes in the $3 \%$ to $5 \%$ range were found to lead to more stable frequency responses. Frequency-watt responses where the power undershoots before settling to the target value were also found to result in increased frequency oscillations if deployed widely. Faster frequency-watt responses were found to be more stable than slower responses over the range simulated, though investigation of possible sub-synchronous interactions was not in the scope of this research.

The effect of frequency-watt control (without power reserve) on the annual energy production of any given DER system is expected to be negligible (less than $0.02 \%$ for the recommended settings) due to the relative rarity and brief duration of overfrequency events. This assumes the deadband chosen lies outside the normal range of frequency variation, which is the case for the recommended deadband setting. In addition, because frequency is largely uniform across an interconnected power system, there should be no outlier customers who experience a larger impact.

Several challenges associated with frequency-watt control of distributed PV are summarized in the next section followed by recommendations for frequency-watt deployment.

\subsection{Challenges of DER-Based Frequency Support}

While enabling frequency-watt control of distributed resources is expected to be beneficial for bulk system stability, several challenges must be overcome to best take advantage of the full capabilities of DER-based frequency support. Those challenges are briefly summarized here. 
Grid operators today typically have no communications with DERs, so it is currently difficult to make changes - as the system continues to evolve - to frequency-watt settings once inverters are placed in service. This could be mitigated by making provisions (both technical and contractual) to remotely modify DER settings in the future.

Grid operators also typically have no way to directly measure the individual or aggregate output of DERs, so they must rely on estimates. Thus, as DER generation varies, the amount of upward or downward response available from distributed PV and storage at any given moment is not known with a high degree of confidence. This could be mitigated by making provisions to monitor distribution-connected, customer-owned resources in the future, or by improving tools for estimating the online DER capacity. Ideally these tools should estimate not just the total capacity, but also the aggregate capacities of legacy PV, PV with partial ride-through, PV with full ride-through, PV with frequency-watt control enabled, and storage with frequency-watt control enabled. For DERs with frequency-watt control enabled, system operators would need to know the droop settings and have a real-time estimate of the aggregate ability to increase or decrease power in response to frequency events.

Acute overfrequency events are typically initiated by the loss of a section of load. Because distributed PV is embedded with load, some amount of PV is typically lost during daytime load rejection events. This loss of PV generation mitigates the overfrequency event to some degree, but it also reduces the amount of downward response available from distributed PV by an unknown amount. This increases the uncertainty and variability in the system frequency response. Likewise, underfrequency load shedding tends to shed a non-negligible amount of DER generation (and the frequency response associated with that generation). This also increases the uncertainty related to DER frequency response.

Bulk power systems are designed to be powered using three-phase generation resources. Distributed PV inverters, especially those on Oahu, are typically single-phase connected, and like single-phase loads, single-phase PV systems are typically not perfectly balanced between phases. Relying on unbalanced resources for essential bulk system services such as frequency response may introduce new challenges, and typical positive sequence modeling tools such as PSS/E may not be appropriate for modeling this. In general, high levels of inverter-based resources will probably eventually require transitioning to EMT-type modeling tools, not just for imbalance, but also to capture relevant fast inverter control dynamics that occur on a sub-cycle timeframe.

Frequency events sometimes occur in association with voltage events, and the uncertain response of distributed $\mathrm{PV}$ to low voltage events contributes to the uncertainty in distributed PV frequency response, and to bulk system challenges in general. For example, fault-induced delayed voltage recovery on the distribution system may cause voltages to remain below $0.5 \mathrm{pu}$ for over 0.6 seconds. This will cause distributed PV to either trip offline or momentarily cease to export current. In the case of momentary cessation, the time for the inverter to return to service following voltage recovery introduces additional uncertainty. Preliminary modeling of these issues performed in this project found that PV responses to voltage faults can have profound consequences for Oahu bulk system operations and stability [25], [26].

Most of the above challenges are exacerbated by the inability of common models and modeling tools to adequately capture both transmission systems and distribution systems in the same simulation. For example, PSS/E and other bulk system transient modeling tools use positivesequence models, so they assume balance between phases and neglect sub-cycle dynamics such as inverter phase-locked loops. While some ongoing work is beginning to develop 
modeling tools that can capture both transmission and distribution dynamics, those modeling tools have limitations, especially with respect to tradeoffs between model fidelity/granularity and simulation time requirements. In addition, even given perfect modeling tools, aggregation of distributed resources and loads is necessary to achieve a tractable model. Accurate models of the frequency and voltage responses of loads and DERs are often not available.

\subsection{Recommendations for Activation of Frequency-Watt: Default Settings and Ranges of Adjustability}

A large aggregate power rating of frequency-responsive DERs is needed to have a measurable effect on grid frequency stability, even in a relatively small power system such as Oahu's.

Because it takes time to build up a base of frequency-responsive DERs, it is recommended that frequency-watt control requirements be implemented expeditiously.

As battery energy storage becomes more common on the distribution system, there is an opportunity to leverage it to provide underfrequency response. The same is true of PV systems, if curtailed for tariff-related reasons. It is recommended that both underfrequency and overfrequency response be required from DERs. However, in alignment with IEEE 1547-2018, it is not recommended to require PV systems to operate with power reserve.

While it is possible to increase the magnitude of the frequency-watt response from distributed PV by increasing the droop slope (i.e. reducing the droop percentage), steeper slopes have been shown to lead to greater oscillations in frequency in some cases, which is undesirable. Thus a moderate droop slope between $5 \%$ and $3 \%$ (inclusive) is recommended. A droop slope equal to that of synchronous generators' governor responses will lead to proportional sharing of the primary frequency response burden, but it may also be desirable to have fast-responding inverter-based resources shoulder more of the PFR burden, especially because they do not provide stabilizing inertial response. The recommended range of adjustability of droop slope allows for a somewhat wider range of droop slopes in both directions to facilitate future adjustments as even larger amounts of DERs are deployed. While the high end of the frequency-watt range of adjustability is above the range specified in IEEE 1547-2018, the standard states that "the upper value is a minimum requirement (the setting may be set to greater values)". Thus the range of adjustability recommended here is allowable per IEEE 1547.

Faster time responses of frequency-watt control can increase the ability of the function to reduce frequency deviations following generation and load loss events. Faster time responses have also been shown to lead to fewer frequency oscillations on the Oahu system, as compared to slower time responses. However, this report has not examined possible interactions between fast inverter responses and conventional generator dynamics, such as sub-synchronous torsional interactions. Before the aggregate rating of frequency-responsive PV inverters becomes large enough to significantly affect the frequency (likely several years in the future), the potential for such interactions should be investigated.

It was found that frequency-watt deadbands ranging from $17 \mathrm{mHz}$ to $50 \mathrm{mHz}$ should have little impact on monthly $\mathrm{PV}$ energy production and should provide effective mitigation of overfrequency events. Hawaiian Electric's SRD V1.1 currently calls for a deadband of $36 \mathrm{mHz}$. The recommendation of this report is to transition to a $17 \mathrm{mHz}$ deadband in conjunction with the activation of frequency-watt response for both underfrequency and overfrequency events. This will provide improved frequency response and to better coordinate with conventional generation, which typically uses a near-zero deadband in Hawaii. 
At the time of the hardware tests described here, most inverters did not follow the form of the frequency-watt function required in IEEE 1547-2018 (which was in development at the time). In addition, time responses of the frequency-watt response varied significantly between inverter models, and the time responses were generally not adjustable, as IEEE 1547 requires. Finally, as noted above, most inverters were not able to perform underfrequency response even if additional power was available. To enable predictable and stabilizing DER frequency responses (and thus greater levels of inverter-based generation), it is strongly recommended that inverter frequency-watt responses be updated to match IEEE 1547-2018 as soon as practical.

Table 17 shows the recommended frequency-watt control parameters from this work. Three changes are recommended relative to the previous interim report:

1. Activate frequency-watt control for underfrequency events (without requiring reserve), not just overfrequency events as previously recommended.

2. Tighten the frequency-watt curve deadband to $17 \mathrm{mHz}$.

3. Require DERs to perform frequency-watt control in a manner that complies with IEEE 1547-2018 (and Hawaiian Electric's SRD).

Table 17: Recommended frequency-watt control settings for overfrequency and underfrequency events

\begin{tabular}{|l|c|c|c|}
\hline \multicolumn{2}{|c|}{ Parameter } & Default value & Range of adjustability \\
\hline Deadband $(\mathrm{Hz})$ & $\begin{array}{l}\text { dboF, } \\
\text { dbuF }\end{array}$ & 0.017 & $0.017-1.0$ \\
\hline Droop slope $(\mathrm{pu})$ & $\begin{array}{c}\text { koF, } \\
\text { kuF }\end{array}$ & 0.04 & $0.02-0.07$ \\
\hline Response time (s) & $\mathrm{t}_{\mathrm{R}}$ & 0.5 & $0.05-3.0$ \\
\hline
\end{tabular}

These settings should be implemented in a timely manner, while also minimizing impact on DER installers and manufacturers to the extent possible.

Much of this report focuses on the Oahu island power system. While many aspects of this work may apply to some extent in other Hawaii power systems (and other power systems in general), other Hawaiian islands have not been studied as part of this project. Because it will take some time to build up a base of frequency responsive inverters, grid planners may want to consider implementing this report's recommendations in the near future while simultaneously taking the necessary steps to ensure they will have the desired effect (and no unmanageable side effects) on other power systems.

Oahu will rely heavily on distributed PV to meet its renewable portfolio standard goals, so the island's power system is expected to change dramatically over the next several years. As synchronous generators are displaced, distributed PV will likely play a bigger role in providing frequency stability to Oahu's power system, so new settings and/or functionality may be required. 
One project partner commented that it may be possible to remotely enable frequency-watt (asis) in a large number of existing distributed PV systems. It may also be possible to enable frequency-watt control in existing multi-megawatt PV systems. These steps would likely be beneficial, but should be considered carefully, as they may lead the Oahu system to more quickly reach a level of frequency-watt enabled $\mathrm{PV}$ where unintended dynamics are possible. 


\section{References}

[1] Y. Liu, S. You, J. Tan, Y. Zhang, and Y. Liu, "Frequency Response Assessment and Enhancement of the U.S. Power Grids Toward Extra-High Photovoltaic Generation Penetrations-An Industry Perspective," IEEE Trans. Power Syst., vol. 33, no. 3, pp. 34383449, May 2018.

[2] A. Hoke and D. Maksimovic, "Active power control of photovoltaic power systems," in 2013 1st IEEE Conference on Technologies for Sustainability (SusTech), 2013, pp. 70-77.

[3] J. Neely, J. Johnson, J. Delhotal, S. Gonzalez, and M. Lave, "Evaluation of PV FrequencyWatt Function for Fast Frequency Reserves," in Applied Power Electronics Conference, 2016.

[4] A. Hoke et al., "The Frequency-Watt Function: Simulation and Testing for the Hawaiian Electric Companies," National Renewable Energy Laboratory, NREL/TP-5D00-68884, Jul. 2017.

[5] J. Aho et al., "A tutorial of wind turbine control for supporting grid frequency through active power control," in American Control Conference (ACC), 2012, 2012, pp. 3120-3131.

[6] "VDE-AR-N 4105:2011-08 Power generation systems connected to the low-voltage distribution network." VDE, 08-Jan-2011.

[7] "IEEE 1547-2018 - IEEE Standard for Interconnection and Interoperability of Distributed Energy Resources with Associated Electric Power Systems Interfaces," IEEE, Apr. 2018.

[8] C. Vartanian, R. Bauer, L. Casey, C. Loutan, D. Narang, and V. Patel, "Ensuring System Reliability: Distributed Energy Resources and Bulk Power System Considerations," IEEE Power Energy Mag., vol. 16, no. 6, pp. 52-63, Nov. 2018.

[9] "UL 1741 Supplement SA: Grid Support Utility Interactive Inverters and Converters," Underwriters Laboratories, 2016.

[10]"Hawaiian Electric Companies Grid Support Utility-Interactive Advanced Inverter Standards Source Requirements Document for Certification with Underwriters Laboratories 1741 Supplement SA - Version 1.1." Hawaiian Electric Company, Inc., 26-Sep-2017.

[11]B. O'Neill and V. Gevorgian, "PV Controls Utility-Scale Demonstration Project," presented at the UVIG Fall Technical Workshop, 14-Oct-2015.

[12]B. I. Crăciun, T. Kerekes, D. Séra, and R. Teodorescu, "Frequency Support Functions in Large PV Power Plants With Active Power Reserves," IEEE J. Emerg. Sel. Top. Power Electron., vol. 2, no. 4, pp. 849-858, Dec. 2014.

[13]"Primary Frequency Response (PFR)/Fast Frequency Response (FFR) Assessment," presented at the ERCOT FAST Workshop, 28-Mar-2014.

[14]Hawaiian Electric Companies, "Power Supply Improvement Plan," 23-Dec-2016. [Online]. Available: https://www.hawaiianelectric.com/clean-energy-hawaii/integrated-gridplanning/power-supply-improvement-plan. [Accessed: 02-Jun-2019].

[15]A. Hoke, S. Chakraborty, M. Shirazi, E. Muljadi, and D. Maksimovic, "Rapid active power control of photovoltaic systems for grid frequency support," IEEE J. Emerg. Sel. Top. Power Electron., Feb. 2017.

[16]"WECC Criterion PRC-001-WECC-CRT-1.2: Governor Droop Setting." Western Electricity Coordinating Council, 17-May-2016.

[17]P. Gotseff, N. Wunder, A. Hoke, E. Ifuku, and R. Ueda, "Residential Advanced Photovoltaic Inverter Pilot Study Results for Select Distribution Secondaries in Hawai'i," in 2018 IEEE PVSC, 2018, pp. 1418-1423.

[18]J. I. Giraldez Miner et al., "Advanced Inverter Voltage Controls: Simulation and Field Pilot Findings," National Renewable Energy Laboratory, NREL/TP--5D00-72298, Oct. 2018.

[19]R. Thiagarajan, P. Gotseff, A. Hoke, and E. Ifuku, "Inverter testing for verification of Hawaiian Electric Rule 14H," presented at the IEEE PVSC, 2019. 
[20]D. Pattabiraman, J. Tan, V. Gevorgian, A. Hoke, C. Antonio, and D. Arakawa, "Impact of Frequency-Watt Control on the Dynamics of a High DER Penetration Power System," in IEEE Power and Energy Society General Meeting (PESGM), 2018.

[21]Debasish Mondal, Abhijit Chakrabarti, and Aparajita Sengupta, Power System Small Signal Stability Analysis and Control. Elsevier, 2014.

[22]M. J. Erickson, "Improved Power Control of Inverter Sources in Mixed-Source Microgrids," University of Wisconsin, Madison, 2013.

[23]M. Elkhatib, J. Neely, and J. Johnson, "Evaluation of Fast-Frequency Support Functions in High Penetration Isolated Power Systems," in Photovoltaic Specialist Conference (PVSC), 2017.

[24]A. Yazdani and R. Iravani, Voltage-Sourced Converters in Power Systems : Modeling, Control, and Applications. Wiley, 2010.

[25]B. J. Pierre, M. E. Elkhatib, and A. Hoke, "PV Inverter Fault Response Including Momentary Cessation, Frequency-Watt, and Virtual Inertia," in 2018 IEEE 7th World Conference on Photovoltaic Energy Conversion (WCPEC) (A Joint Conference of 45th IEEE PVSC, 28th PVSEC 34th EU PVSEC), 2018, pp. 3660-3665.

[26]B. J. Pierre, M. E. Elkhatib, and A. Hoke, "Photovoltaic Inverter Momentary Cessation: Recovery Process is Key," in IEEE PVSC, 2019.

[27]A. Nelson et al., "Hawaiian Electric Advanced Inverter Grid Support Function Laboratory Validation and Analysis," National Renewable Energy Lab. (NREL), Golden, CO (United States), NREL/TP-5D00-67485, Dec. 2016.

[28]E. Ela, V. Gevorgian, A. Tuohy, B. Kirby, M. Milligan, and M. O'Malley, "Market Designs for the Primary Frequency Response Ancillary Service - Part II: Case Studies," IEEE Trans. Power Syst., vol. 29, no. 1, pp. 432-440, Jan. 2014.

[29]A. Hoke, S. Chakraborty, and T. Basso, "A Power Hardware-in-the-loop Framework for Advanced Grid-interactive Inverter Testing," in IEEE Innovative Smart Grid Technologies Conference (ISGT), 2015.

[30]A. Nagarajan et al., "Network Reduction Algorithm for Developing Distribution Feeders for Real-time Simulators," in review.

[31]"FERC Order No. 842: Essential Reliability Services and the Evolving Bulk-Power SystemPrimary Frequency Response." Federal Energy Regulatory Commission, 15-Feb-2018.

[32]A. Nelson, G. Martin, and J. Hurtt, "Experimental Evaluation of Grid Support Enabled PV Inverter Response to Abnormal Grid Conditions: Preprint," in Photovoltaic Specialist Conference (PVSC), 2017.

[33]A. Hoke, S. Chakraborty, and T. Basso, "Testing advanced photovoltaic inverters conforming to IEEE standard 1547 - Amendment 1," in Photovoltaic Specialist Conference (PVSC), 2014 IEEE 40th, 2014, pp. 1014-1021. 


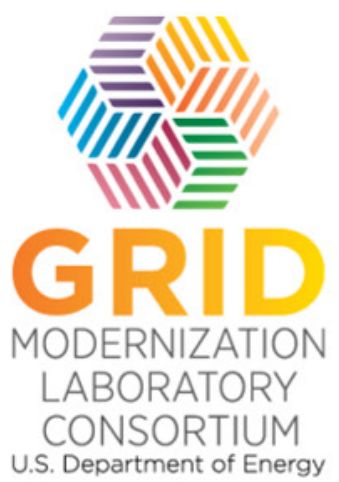

https://gm/c.doe.gov/ 